\title{
Non-Compact Hopf Maps and Fuzzy Ultra-Hyperboloids
}

\author{
Kazuki Hasebe \\ Kagawa National College of Technology, Takuma, Mitoyo, Kagawa 769-1192, Japan \\ hasebe@dg.kagawa-nct.ac.jp
}

February 15, 2018

\begin{abstract}
Fuzzy hyperboloids naturally emerge in the geometries of branes, twistor theory, and higher spin theories. In this work, we perform a systematic study of higher dimensional fuzzy hyperboloids (ultra-hyperboloids) based on non-compact Hopf maps. Two types of non-compact Hopf maps; split-type and hybrid-type, are introduced from the cousins of division algebras. We construct arbitrary even-dimensional fuzzy ultra-hyperboloids by applying the Schwinger operator formalism and indefinite Clifford algebras. It is shown that fuzzy hyperboloids, $H_{F}^{2 p, 2 q}$, are represented by the coset, $H_{F}^{2 p, 2 q} \simeq S O(2 p, 2 q+1) / U(p, q)$, and exhibit two types of generalized dimensional hierarchy; hyperbolic-type (for $q \neq 0$ ) and hybrid-type (for $q=0$ ). Fuzzy hyperboloids can be expressed as fibre-bundle of fuzzy fibre over hyperbolic basemanifold. Such bundle structure of fuzzy hyperboloid gives rise to non-compact monopole gauge field. Physical realization of fuzzy hyperboloids is argued in the context of lowest Landau level physics.
\end{abstract}




\section{Contents}

1 Introduction $\quad 2$

2 Cousins of Quaternions and Indefinite Gamma Matrices 5

2.1 Ultra-hyperboloids . . . . . . . . . . . . . . . . . 5

2.2 Cousins of quaternions and symplectic groups . . . . . . . . . . . 7

2.3 Indefinite gamma matrices . . . . . . . . . . . . . . . . . . 9

2.3.1 $S O(p, q)$ gamma matrices $(p+q$ : even $) \ldots \ldots \ldots \ldots$

2.3.2 $S O(l, m)$ gamma matrices $(l+m$ : odd $)$ from $S O(p, q)(p+q:$ even $) \quad \ldots \quad 11$

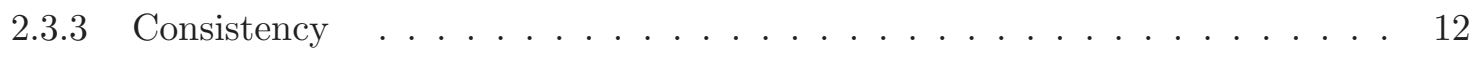

2.3.4 Relations between $S O(1,2 p)$ and $S O(2 p+1)$ gamma matrices . . . . . . 13

3 Non-compact 1st Hopf Map and Fuzzy Two-Hyperboloid 13

3.1 Non-compact 1st Hopf map: $H^{2,0} \simeq H^{2,1} / S^{1} \ldots \ldots \ldots \ldots$

3.2 Fuzzy two-hyperboloid: $H_{F}^{2,0} \ldots \ldots \ldots \ldots \ldots \ldots \ldots$

3.2.1 Finite dimensional non-unitary representation . . . . . . . . . . . . . . . . 15

3.2 .2 Infinite dimensional unitary representation $\ldots \ldots \ldots \ldots$

4 Non-compact 2nd Hopf Maps and Fuzzy Four-Hyperboloids $\quad 17$

4.1 Split 2nd Hopf map: $H^{2,2} \simeq H^{4,3} / H^{2,1} \ldots \ldots \ldots \ldots \ldots$

4.2 Fuzzy split four-hyperboloid: $H_{F}^{2,2} \ldots \ldots \ldots \ldots \ldots \ldots$

4.2.1 Finite dimensional unitary representation . . . . . . . . . . . . . . . . . . . . . . . . . . .

4.2.2 Infinite dimensional unitary representation . . . . . . . . . . . . . . . . 20

4.3 Hybrid 2nd Hopf map: $H^{4,0} \simeq H^{4,3} / S^{3} \ldots \ldots \ldots \ldots \ldots \ldots$

4.4 Fuzzy two-leaf four-hyperboloid: $H_{F}^{4,0}=E A d S_{F}^{4} \ldots \ldots \ldots \ldots$

4.4.1 Finite dimensional non-unitary representation . . . . . . . . . . . . . 23

4.4.2 Infinite dimensional unitary representation . . . . . . . . . . . . . . . . . . . . . . .

4.5 Enhanced Symmetry . . . . . . . . . . . . . . . . . . . . . . . . . . . . . . . . . .

4.5.1 Enhanced algebra and internal structure . . . . . . . . . . . 25

4.5.2 Quantum fluctuations of geometry . . . . . . . . . . . . 28

5 Even Higher Dimensional Generalization of Fuzzy Hyperboloid 29

5.1 Hierarchical structure of fuzzy ultra-hyperboloid . . . . . . . . . . . . . . 29

5.1 Hyperbolic hierarchy: Construction I . . . . . . . . . . . . . . . . . . . . . . . . . .

5.1 .2 Hybrid hierarchy: Construction II . . . . . . . . . . . . . . . . . . . . . . . . . . . . . . .

5.1 .3 Examples in low dimension . . . . . . . . . . . . . . . . 31

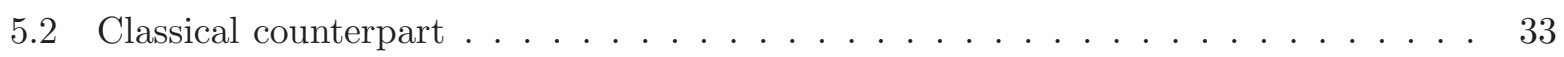

5.3 Non-compact monopoles . . . . . . . . . . . . . . . . . . . . . 34

$5.4 \quad U(1)$ monopole on $H^{2,0} \ldots \ldots \ldots \ldots \ldots \ldots \ldots \ldots \ldots$

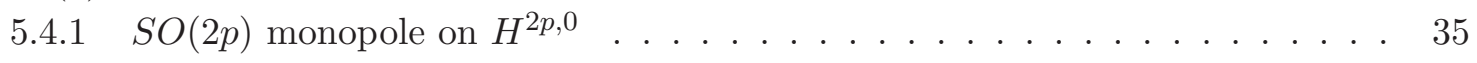

$5.4 .2 S O(2 p, 2 q)$ monopole on $H^{2 p, 2 q} \ldots \ldots \ldots \ldots \ldots \ldots \ldots$ 
6 Fuzzy Hyperboloid realized as Lowest Landau Level 38

6.1 Lowest Landau level on two-hyperboloid . . . . . . . . . . . . . . . . . . 39

6.2 Lowest Landau level on four-hyperboloid . . . . . . . . . . . . . . . . . . . . 40

6.2.1 Lowest Landau level on $H^{2,2}$ in $S U(1,1)$ monopole background . . . . . . . 40

6.2.2 $U(1)$ monopole gauge fields on $\mathbb{C} P^{1,2} \ldots \ldots \ldots \ldots \ldots$. . . . . . . . . . . . . . . . 42

6.2.3 Lowest Landau level on $\mathbb{C} P^{1,2}$ in $U(1)$ monopole background . . . . . . . . 43

7 Summary and Discussions $\quad 44$

A Hybrid 3rd Hopf map $\quad 45$

B Split Algebra and Fuzzy Split-Hyperboloid $\quad 48$

B.1 Fuzzy two-hyperboloid: $\mathcal{H}_{F}^{1,1} \ldots \ldots \ldots \ldots \ldots \ldots \ldots$

B.2 Fuzzy four-hyperboloid: $\mathcal{H}_{F}^{2,2} \ldots \ldots \ldots \ldots \ldots \ldots \ldots$

B.3 Fuzzy split-hyperboloids: $\mathcal{H}_{F}^{p, p} \ldots \ldots \ldots \ldots \ldots$

C Indefinite Complex Projective Spaces $\quad 52$

C.1 Indefinite complex projective spaces . . . . . . . . . . . . . . . . 52

C.2 Split-complex projective spaces . . . . . . . . . . . . . . . 52

\section{Introduction}

Fuzzy geometry has been an active research field in the past decades. Fuzzy geometry naturally introduces a cut-off in small scale, which softens UV divergence of field theory. A most typical and well understood fuzzy manifold is fuzzy (two-)sphere $[1,2,3]$. Fuzzy two-sphere and its higher dimensional cousins naturally arise as non-trivial classical solutions of matrix model of string theory (as a review, see Ref.[4] for instance and references therein). Interestingly, the original symmetry of classical manifolds is generally enhanced to be a larger symmetry in their corresponding fuzzy manifolds. Such symmetry enhancement is interpreted as quantum geometrical effect particular to fuzzy manifolds $[5,6]$ : quantum fluctuations on fuzzy manifold "smear" the original classical geometry to generate a larger fuzzy geometry. Fuzzy spheres have mathematical structures closely related to Clifford algebra. The coordinates on fuzzy $2 p$-sphere correspond to $S O(2 p+1)$ gamma matrices of fully symmetric representation [7]. A most convenient way to handle the fully symmetric representation is to adopt the Schwinger operator formalism ${ }^{1}$ : the fuzzy coordinates of fuzzy two-spheres $[8,9]$ and four-spheres [10] are constructed by sandwiching the $S O(3)$ and $S O(5)$ gamma matrices by the Schwinger operators. Similarly, the coordinates of even higher dimensional fuzzy spheres can be constructed[11, 12, 13, 14, 15]. Furthermore, supersymmetric generalizations of fuzzy spheres $[6,16,17]$ and general fuzzy Riemann surfaces [18] have also been explored.

\footnotetext{
${ }^{1}$ The Schwinger operator formalism is sometimes referred to as oscillator formalism or oscillator realization in literature.
} 
The Schwinger construction of the fuzzy spheres is regarded as an operator version of the Hopf maps (see Ref.[19] as a review, and Refs.[20, 21, 22]). The Hopf maps are topological maps from sphere to sphere in different dimensions, introduced by Heinz Hopf about eighty years ago [23, 24]:

$$
S^{15} \stackrel{S^{7}}{\longrightarrow} \underset{S^{8}}{\longrightarrow} S^{4} S^{S^{3}} \stackrel{S^{1}}{\longrightarrow} S^{2}
$$

The Hopf maps (which we designate as the compact Hopf maps) are related to the division algebras, i.e. complex number (1st), quaternions (2nd) and octonions (3rd) [19, 25]. Quaternions and octonions are constructed by applying Cayley-Dickson construction to complex number [26]. The geometry of the Hopf maps reflects such particular construction and exhibits a hierarchical structure as shown in the above picture. Meanwhile, Clifford algebra is another generalization of the complex number, and the coordinates of higher dimensional fuzzy spheres are essentially regarded as gamma matrices of orthogonal groups. As is well known, higher dimensional Clifford algebra is constructed from lower dimensional one. This particular structure of construction of gamma matrices brings a hierarchical geometry to fuzzy spheres [12, 27].

A main goal of this work is to explore a systematic construction of higher dimensional fuzzy hyperboloids (fuzzy ultra-hyperboloids). Fuzzy hyperboloids are typical curved fuzzy manifolds that are regarded as non-compact analogue of fuzzy spheres. They arise as classical solutions of the matrix model on a pp-wave background geometry [28, 29], and also play a crucial role in the discussion of $\mathrm{UV} / \mathrm{IR}$ connection [30, 31]. Furthermore, in the context of twistor theory $[32,33,34,35]$ and higher spin theory [36, 37], fuzzy hyperboloids naturally emerge as their underlying geometry. Though low dimensional fuzzy hyperboloids are fairly well investigated $[1,38,39,40,41]$, studies of fuzzy ultra-hyperboloids have a rather short history $[42,43,44]$. We show that the close relations between fuzzy spheres and the Hopf maps can naturally be extended in the construction of fuzzy ultra-hyperboloids. It is shown that there are two kinds of non-compact Hopf maps: the first is the split-type (split Hopf maps) constructed by the split algebras $[45,46]^{2}$ :

$$
H^{8,7} \stackrel{H^{4,3}}{\underset{H^{4,4}}{\longrightarrow}} \underset{H^{2,2}}{\stackrel{H^{1,0}}{\longrightarrow}} H^{1,1}
$$

The other is the hybrid type (hybrid Hopf maps ${ }^{3}$ ):

\footnotetext{
${ }^{2}$ See also the preceding literatures $[47,48]$ for the 1 st and 2 nd split Hopf maps.

${ }^{3}$ The hybrid Hopf maps are a hybridization of the compact and split Hopf maps in the sense that the total manifolds are same as those of the split Hopf maps and the fibres are those of the compact Hopf maps.
} 


$$
\begin{array}{rlll} 
& H^{2,1} & \stackrel{S^{1}}{\longrightarrow} & H^{2,0} \\
H^{4,3} & \stackrel{S^{3}}{\longrightarrow} H^{4,0} & \\
H^{8,7} & \stackrel{S^{7}}{\longrightarrow} & H^{8,0} &
\end{array}
$$

With these non-compact Hopf maps, we argue geometrical structures of low dimensional fuzzy hyperboloids ${ }^{4}$. In general, the coordinates on fuzzy hyperboloids are given by gamma matrices of indefinite orthogonal groups. Corresponding to representations of the non-compact groups, two formulations have been proposed to realize fuzzy hyperboloids (see Ref.[44] and references therein); one of which is to adopt unitary infinite dimensional representation of non-compact group, and the other is to adopt the non-unitary finite dimensional representation. We address their relations in the Schwinger operator formalism. As the hierarchical structure of the gamma matrices reflects the dimensional hierarchy of fuzzy spheres, generalized dimensional hierarchies are observed in their non-compact counterparts. As coset, fuzzy spheres are given by [7]

$$
S_{F}^{2 p} \simeq S O(2 p+1) / U(p)
$$

which are locally expressed as $[11,12]$ :

$$
S_{F}^{2 p} \sim S^{2 p} \otimes S_{F}^{2 p-2}
$$

Here, $\sim$ signifies local equivalence (throughout the paper, we adopt $\sim$ to express local equivalence): $S_{F}^{2 p}$ is locally, but not globally, equivalent to the trivial fibration of $S_{F}^{2 p-2}$ over the basemanifold $S^{2 p}$. Thus, fuzzy sphere $S_{F}^{2 p}$ can be regarded as a "twisted" fuzzy fibre-bundle of fuzzy fibre $S_{F}^{2 p-2}$ over basemanifold $S^{2 p}$. For fuzzy hyperboloids, we have

$$
H_{F}^{2 p, 2 q} \simeq S O(2 p, 2 q+1) / U(p, q) .
$$

Corresponding to the split and hybrid Hopf maps, the fuzzy hyperboloids exhibit two types of the dimensional hierarchy, one of which is the hyperbolic type

$$
H_{F}^{2 p, 2 q} \sim H^{2 p, 2 q} \otimes H_{F}^{2 p, 2 q-2}(q \neq 0),
$$

and the other is the hybrid type

$$
H_{F}^{2 p, 0} \sim H^{2 p, 0} \otimes S_{F}^{2 p-2} .
$$

That is, $H_{F}^{2 p, 2 q}$ is locally equivalent to fibre-bundle of fibre $H_{F}^{2 p, 2 q-2}$ over basemanifold $H^{2 p, 2 q}$, and similarly $H_{F}^{2 p, 0}$ is equivalent to fibre-bundle of $S_{F}^{2 p-2}$ over $H^{2 p, 0}$. In either cases, the connection of fuzzy fibre gives rise to (non-compact) monopole gauge field on hyperboloid ${ }^{5}$. As the lowest

\footnotetext{
${ }^{4}$ Interestingly, the compact [49,50] and split Hopf maps [51] are also related to entangled qubit geometry of quantum information, and black hole physics [52]. The quaternionic and split quaternionic gauge fields have also been used to construct a generalized Chern-Simons theory [53, 54].

${ }^{5}$ Such non-compact monopole and corresponding algebraic structure (split-quaternions) play important roles in pseudo-hermitian quantum mechanics $[55,56,57]$ and topological phase of non-hermitian systems $[58,59,60]$.
} 
Landau level physics in such monopole background, a physical interpretation of fuzzy hyperboloids is provided, too.

This paper is organized as follows. In Section 2, we review some basic mathematics of quaternions, symplectic groups, and indefinite gamma matrices. In Section 3, we present close relations between the non-compact 1st Hopf map and the Schwinger operator construction of fuzzy twohyperboloid. We extend the discussions to four-dimensional fuzzy hyperboloids with use of the non-compact 2nd Hopf maps in Section 4. We further discuss construction of even higher dimensional fuzzy hyperboloids based on gamma matrices of indefinite orthogonal groups in Section 5 . In Section 6, we demonstrate a physical realization of fuzzy hyperboloid in the context of lowest Landau level physics. Section 7 is devoted to summary and discussions.

\section{Cousins of Quaternions and Indefinite Gamma Matrices}

In this section, we give a brief introduction of ultra-hyperboloids (Section 2.1), cousins of quaternions (Section 2.2), and indefinite Clifford algebras (Section 2.3).

\subsection{Ultra-hyperboloids}

The coordinates of ultra-hyperboloid $H^{p, q}, x^{i}(i=1,2, \cdots, p)$ and $y^{j}(j=1,2, \cdots, q+1)$, are defined so as to satisfy

$$
\sum_{i=1}^{p} x^{i} x^{i}-\sum_{j=1}^{q+1} y^{j} y^{j}=-1 .
$$

Note $H^{p . q} \neq H^{q, p}(p \neq q)$. Ultra-hyperboloids are expressed by the following $\operatorname{cosets}^{6}$ :

$$
\begin{aligned}
& H^{p, q} \simeq S O(p, q+1) / S O(p, q) \simeq S O(q+1, p) / S O(q, p), \\
& H^{2 p, 2 q+1} \simeq S U(p, q+1) / S U(p, q) \simeq S U(q+1, p) / S U(q, p), \\
& H^{4 p, 4 q+3} \simeq S p(p, q+1) / S p(p, q) \simeq S p(q+1, p) / S p(q, p) .
\end{aligned}
$$

Topology of $H^{p, q}$ is given by

$$
H^{p, q} \simeq R^{p} \otimes S^{q}
$$

and then,

$$
\pi_{q}\left(H^{p, q}\right)=\pi_{q}\left(S^{q}\right)=\mathbb{Z}
$$

Several examples are described as

$$
\begin{aligned}
& S^{p} \equiv H^{0, p} \\
& d S^{p} \equiv H^{1, p-1} \simeq R^{1} \otimes S^{p-1} \\
& A d S^{p} \equiv H^{p-1,1} \simeq R^{p-1} \otimes S^{1} \\
& E A d S^{p}\left(=H^{p}\right) \equiv H^{p, 0} \simeq R^{p} \otimes Z_{2},
\end{aligned}
$$

\footnotetext{
${ }^{6}$ With $S^{p} \simeq H^{0, p}$, one may readily see that Eq.(7) reproduces the coset realizations of sphere: $S^{p} \simeq S O(p+$ 1) $/ S O(p), S^{2 p+1} \simeq S U(p+1) / S U(p)$, and $S^{4 p+3} \simeq S p(p+1) / S p(p)$.
} 
where $S^{p}, d S^{p}, A d S^{p}$ and $E A d S^{p}$ denote $p$-dimensional sphere, de Sitter, anti-de Sitter and Euclidean anti-de Sitter spaces, respectively. Note $d S^{2}=A d S^{2}\left(=H^{1,1}\right) . S^{p}(p>2), d S^{p}(p>2)$, and $E A d S^{p}$ are simply connected manifolds:

$$
\pi_{1}\left(S^{p>2}\right) \simeq \pi_{1}\left(d S^{p>2}\right) \simeq \pi_{1}\left(E A d S^{p}\right) \simeq 1
$$

$E A d S^{p}$ denotes $p$-dimensional two-leaf hyperboloid. $\left(Z_{2}\right.$ in (10d) corresponds to the two-leaves.) $d S^{p}$ is one-leaf hyperboloid. $A d S^{p}$ are connected but not simply connected:

$$
\pi_{1}\left(A d S^{p}\right) \simeq \mathbb{Z}
$$

As described in Introduction, the basemanifolds of the compact, non-compact, and hybrid Hopf maps are respectively given by

$\begin{array}{lllc} & \text { Compact } & \text { Split } & \text { Hybrid } \\ \text { (1st) } & H^{0,2}=S^{2} & H^{1,1} & H^{2,0}=E A d S^{2} \\ \text { (2nd) } & H^{0,4}=S^{4} & H^{2,2} & H^{4,0}=E A d S^{4} \\ \text { (3rd) } & H^{0,8}=S^{8} & H^{4,4} & H^{8,0}=E A d S^{8}\end{array}$

The hybrid Hopf maps represent trivial fibration, since the basemanifolds, $H^{2,0}, H^{4,0}$ and $H^{8,0}$, are two-leaf hyperboloids. The symmetry groups of the basemanifolds are

$\begin{array}{lccc} & \text { Compact } & \text { Split } & \text { Hybrid } \\ (1 \mathrm{st}) & S O(3) & S O(2,1) & S O(1,2) \\ (2 \mathrm{nd}) & S O(5) & S O(3,2) & S O(1,4) \\ (3 \mathrm{rd}) & S O(9) & S O(5,4) & S O(1,8)\end{array}$

The symmetry groups of 1 st and 2nd Hopf maps are compactly restated by quaternion and splitquaternion groups:

$\begin{array}{cccc} & \text { Compact } & \text { Split } & \text { Hybrid } \\ (1 \text { st }) & U(1 ; \mathbb{H}) & U\left(1 ; \mathbb{H}^{\prime}\right) & U\left(1 ; \mathbb{H}^{\prime}\right) \\ (2 \mathrm{nd}) & U(2 ; \mathbb{H}) & U\left(2 ; \mathbb{H}^{\prime}\right) \simeq U\left(1,1 ; \mathbb{H}^{\prime}\right) & U(1,1 ; \mathbb{H})\end{array}$

Here, $\mathbb{H}$ and $\mathbb{H}^{\prime}$ respectively denote quaternion and split-quaternion. Note that $U(p, q ; \mathbb{H})$ and $U\left(p, q ; \mathbb{H}^{\prime}\right)(p+q \leq 2)$ all appear in this table. See also Eqs.(25) and (26). 


\subsection{Cousins of quaternions and symplectic groups}

Quaternions and their cousins $\left(1, q_{1}, q_{2}, q_{3}\right)$ are defined so as to satisfy

$$
q_{i} q_{j}=-q_{j} q_{i}(i \neq j), \quad q_{1} q_{2} q_{3}=-1
$$

and

$$
\left(q_{1}\right)^{2}=\epsilon_{1}, \quad\left(q_{2}\right)^{2}=\epsilon_{2}, \quad\left(q_{3}\right)^{2}=\epsilon_{3},
$$

where each of $\epsilon_{1}, \epsilon_{2}$ and $\epsilon_{3}$ takes either +1 or -1 . Then, there are four types of quaternions:

- Quaternions [61]: all of $\epsilon_{1}, \epsilon_{2}, \epsilon_{3}$ in (14) are -1 .

- "Hybrid" quaternions ${ }^{7}$ : two of $\epsilon_{1}, \epsilon_{2}, \epsilon_{3}$ are -1 and the remaining is +1 .

- Split quaternions [62]: two of $\epsilon_{1}, \epsilon_{2}, \epsilon_{3}$ are +1 and the remaining is -1 .

- Hyperbolic quaternions [63]: all of $\epsilon_{1}, \epsilon_{2}, \epsilon_{3}$ are +1 .

$q_{1}, q_{2}$ and $q_{3}$ are generalization of imaginary unit and called imaginary quaternions, and their conjugation is given by

$$
q_{1}^{*}=-q_{1}, \quad q_{2}{ }^{*}=-q_{2}, \quad q_{3}^{*}=-q_{3} .
$$

With four real parameters, $r_{0}, r_{1}, r_{2}, r_{3}$, an arbitrary quaternion number is constructed as

$$
q=r_{0}+r_{1} q_{1}+r_{2} q_{2}+r_{3} q_{3}
$$

Similarly, arbitrary hybrid, split and hyperbolic quaternions are respectively constructed by replacing $q_{i}(i=1,2,3)$ in (16) with hybrid, split and hyperbolic imaginary quaternions. The conjugate of $q$ is given by

$$
q^{*}=r_{0}-r_{1} q_{1}-r_{2} q_{2}-r_{3} q_{3},
$$

and $q^{*} q$ is derived as

$$
q^{*} q=q q^{*}=r_{0}^{2}-\epsilon_{1} r_{1}^{2}-\epsilon_{2} r_{2}^{2}-\epsilon_{3} r_{3}^{2}
$$

with $\epsilon_{1}, \epsilon_{2}, \epsilon_{3}$ (14). Thus, for quaternions, (18) provides the inner product in Euclidean space. Similarly, (18) respectively yields the inner product in split (signature) space for split quaternions, and Lorentzian (signature) space for both hybrid and hyperbolic quaternions. It may be worthwhile to write down the algebra of four-types of quaternions explicitly:

- Quaternions:

$$
\begin{aligned}
& \left(q_{1}\right)^{2}=\left(q_{2}\right)^{2}=\left(q_{3}\right)^{2}=-1, \\
& q_{1} q_{2}=-q_{2} q_{1}=q_{3}, \quad q_{2} q_{3}=-q_{3} q_{2}=q_{1}, \quad q_{3} q_{1}=-q_{1} q_{3}=q_{2} .
\end{aligned}
$$

\footnotetext{
${ }^{7}$ The author does not know how this type of quaternions is called in literature. In this paper, we call this type of quaternions hybrid quaternions, since they are related to the hybrid Hopf maps. The hybrid quaternions do not respect associativity like hyperbolic quaternions. However, in both cases, $q_{1} q_{2} q_{3}$ in (13) is defined to be independent on the associative order, i.e. $\left(q_{1} q_{2}\right) q_{3}=q_{1}\left(q_{2} q_{3}\right)$.
} 
- Hybrid quaternions:

$$
\begin{aligned}
& \left(q_{1}\right)^{2}=+1, \quad\left(q_{2}\right)^{2}=\left(q_{3}\right)^{2}=-1, \\
& q_{1} q_{2}=-q_{2} q_{1}=q_{3}, \quad q_{2} q_{3}=-q_{3} q_{2}=-q_{1}, \quad q_{3} q_{1}=-q_{1} q_{3}=q_{2} .
\end{aligned}
$$

- Split quaternions:

$$
\begin{aligned}
& \left(q_{1}\right)^{2}=\left(q_{2}\right)^{2}=+1, \quad\left(q_{3}\right)^{2}=-1, \\
& q_{1} q_{2}=-q_{2} q_{1}=q_{3}, \quad q_{2} q_{3}=-q_{3} q_{2}=-q_{1}, \quad q_{3} q_{1}=-q_{1} q_{3}=-q_{2} .
\end{aligned}
$$

- Hyperbolic quaternions:

$$
\begin{aligned}
& \left(q_{1}\right)^{2}=\left(q_{2}\right)^{2}=\left(q_{3}\right)^{2}=+1, \\
& q_{1} q_{2}=-q_{2} q_{1}=-q_{3}, \quad q_{2} q_{3}=-q_{3} q_{2}=-q_{1}, \quad q_{3} q_{1}=-q_{1} q_{3}=-q_{2} .
\end{aligned}
$$

One may find that in either type of quaternions, $q_{1} q_{2} q_{3}=\left(q_{1} q_{2}\right) q_{3}=q_{1}\left(q_{2} q_{3}\right)=-1$ (13) holds.

Replacing the imaginary unit of the Pauli matrices with imaginary quaternions, we have

$$
\begin{aligned}
\sigma^{2} & =\left(\begin{array}{cc}
0 & -i \\
i & 0
\end{array}\right), \quad \sigma^{1}=\left(\begin{array}{ll}
0 & 1 \\
1 & 0
\end{array}\right), \quad \sigma^{3}=\left(\begin{array}{cc}
1 & 0 \\
0 & -1
\end{array}\right) \\
\rightarrow \quad \gamma^{1} & =\left(\begin{array}{cc}
0 & -q_{1} \\
q_{1} & 0
\end{array}\right), \quad \gamma^{2}=\left(\begin{array}{cc}
0 & -q_{2} \\
q_{2} & 0
\end{array}\right), \quad \gamma^{3}=\left(\begin{array}{cc}
0 & -q_{3} \\
q_{3} & 0
\end{array}\right), \quad \gamma^{4}=\left(\begin{array}{cc}
0 & 1 \\
1 & 0
\end{array}\right), \quad \gamma^{5}=\left(\begin{array}{cc}
1 & 0 \\
0 & -1
\end{array}\right) .
\end{aligned}
$$

They satisfy the anti-commutation relations of $S O(5)$ gamma matrices, $\left\{\gamma^{a}, \gamma^{b}\right\}=2 \eta^{a b}$, with $\eta^{a b}=\operatorname{diag}(+,+,+,+,+)$. When we adopt the hybrid, split, and hyperbolic quaternions instead of quaternions in (23), $\gamma^{a}$ satisfy the anti-commutation relations with $\eta^{a b}=\operatorname{diag}(-,+,+,+,+)$, $\eta^{a b}=\operatorname{diag}(-,-,+,+,+)$ and $\eta^{a b}=\operatorname{diag}(-,-,-,+,+)$, respectively. Thus, we obtain $S O(4,1)$, $S O(3,2)$ and $S O(2,3)$, gamma matrices for hybrid, split and hyperbolic quaternions, respectively:

- Quaternions

- Hybrid quaternions

- Split quaternions

- Hyperbolic quaternions $\rightarrow S O(2,3)$ gamma matrices

$$
\rightarrow S O(5) \text { gamma matrices }
$$$$
\rightarrow \quad S O(4,1) \text { gamma matrices }
$$

$\rightarrow \quad S O(3,2)$ gamma matrices

The $S O(5)$ and $S O(3,2)$ gamma matrices are crucial in constructing the 2nd compact [64] and split Hopf maps [45]. Then, one may expect that $S O(4,1)$ gamma matrices play a similar role in constructing the 2 nd hybrid Hopf map ${ }^{8}$. This expectation turns out to be true in Section 4.3. Note, however, there is a crucial difference: hybrid quaternions (and also hyperbolic quaternions)

\footnotetext{
${ }^{8}$ Meanwhile, since the $S O(2,3)$ gamma matrices of hyperbolic quaternions are equivalent to $S O(3,2)$ gamma matrices of split quaternions up to imaginary unit, we do not consider the $S O(2,3)$ case.
} 
do not respect associativity unlike quaternions and split-quaternions, and hence hybrid quaternions cannot be realized by matrices ${ }^{9}$. For instance, from (20) we find a non-associative relation:

$$
\left(q_{1} q_{1}\right) q_{2} \neq q_{1}\left(q_{1} q_{2}\right)
$$

The left-hand side is $\left(q_{1} q_{1}\right) q_{2}=(+1) q_{2}=q_{2}$, while the right-hand side is $q_{1}\left(q_{1} q_{2}\right)=q_{1} q_{3}=$ $-q_{2}$. Thus, among the cousins of quaternions, only the original and split-quaternions satisfy associative algebras, and their groups are consistently defined. Low dimensional quaternion and split-quaternions groups, $U(p, q ; \mathbb{H})$ and $U\left(p, q ; \mathbb{H}^{\prime}\right)(p+q \leq 2)$, are all exhausted as

$$
\begin{aligned}
& U(1 ; \mathbb{H}) \equiv S p(1) \simeq U S p(2) \simeq S U(2) \simeq S O(3), \\
& U\left(1 ; \mathbb{H}^{\prime}\right) \simeq S p(2 ; \mathbb{R}) \simeq S U(1,1) \simeq S O(2,1) \simeq S O(1,2),
\end{aligned}
$$

and

$$
\begin{aligned}
& U(2 ; \mathbb{H}) \equiv S p(2) \simeq U S p(4) \simeq S O(5) \\
& U\left(2 ; \mathbb{H}^{\prime}\right) \simeq U\left(1,1 ; \mathbb{H}^{\prime}\right) \simeq S p(4 ; \mathbb{R}) \simeq S O(3,2) \simeq S O(2,3), \\
& U(1,1 ; \mathbb{H}) \equiv S p(1,1) \simeq U S p(2,2) \simeq S O(4,1) \simeq S O(1,4) .
\end{aligned}
$$

Note that $S O(5), S O(3,2)$ and $S O(4,1)$ structures naturally appear in $U(p, q ; \mathbb{H})$ and $U\left(p, q ; \mathbb{H}^{\prime}\right)$ for $p+q=2(26)$. Low dimensional unitary groups of quaternion and split-quaternions provide basic examples of indefinite orthogonal groups.

\subsection{Indefinite gamma matrices}

Here, we introduce general indefinite orthogonal groups and their gamma matrices. In indefinite orthogonal groups, finite dimensional representation of their gamma matrices is generally given by non-hermitian matrix. Multiplied by a suitable matrix, non-hermitian gamma matrices are transformed to hermitian matrices. We mainly discuss such "hermitianization" of gamma matrices of indefinite orthogonal groups, which will play a crucial role in explicit construction of the noncompact Hopf maps. For detail properties of indefinite gamma matrices, readers may consult Ref.[65].

\subsection{1 $S O(p, q)$ gamma matrices $(p+q$ : even $)$}

First, we consider even dimensional space-times:

$$
p+q: \text { even, }
$$

where $S O(p, q)$ gamma matrices, $\gamma^{\mu}(\mu=1,2, \cdots, p+q)$, satisfy

$$
\left\{\gamma^{\mu}, \gamma^{\nu}\right\}=2 \eta^{\mu \nu}
$$

\footnotetext{
${ }^{9}$ It is well known that the quaternions are represented by (imaginary unit times) $S U(2)$ Pauli matrices, and similarly the split-quaternions are by (imaginary unit times) $S U(1,1)$ Pauli matrices (see Section 3.1 ).
} 
with

$$
\eta^{\mu \nu}=\operatorname{diag}(\overbrace{+,+, \cdots,+}^{p}, \overbrace{-,-, \cdots,-}^{q}) .
$$

Since $\left(\gamma^{\mu}\right)^{2}=+1, \gamma^{\mu}(\mu=1,2, \cdots, p)$ may be taken as hermitian matrices. On the other hand, since $\left(\gamma^{\mu}\right)^{2}=-1, \gamma^{\mu}(\mu=p+1, p+2, \cdots, p+q)$ may be taken as anti-hermitian matrices [65]. Thus, the $S O(p, q)$ gamma matrices are classified into hermitian and anti-hermitian matrices. We have two different (hermitian and anti-hermitian) matrices that hermitianize the gamma matrices. One hermitianizing matrix is constructed by multiplying all of the anti-hermitian gamma matrices

$$
k=(i)^{\frac{1}{2} q(q-1)} \gamma^{p+1} \gamma^{p+2} \cdots \gamma^{p+q},
$$

and the other is by multiplying the remaining all hermitian gamma matrices ${ }^{10}$

$$
k^{\prime}=(i)^{\frac{1}{2} p(p+1)+1} \gamma^{1} \gamma^{2} \cdots \gamma^{p}
$$

With (28), it is straightforward to show that $k \gamma^{\mu}$ and $k^{\prime} \gamma^{\mu}$ are indeed hermitian

$$
\begin{aligned}
& \left(k \gamma^{\mu}\right)^{\dagger}=k \gamma^{\mu}, \\
& \left(k^{\prime} \gamma^{\mu}\right)^{\dagger}=k^{\prime} \gamma^{\mu} .
\end{aligned}
$$

Thus, all of the gamma matrices of $S O(p, q)$ are hermitianized multiplied by either of $k$ and $k^{\prime}$. Hermitian conjugates of $k$ and $k^{\prime}$ are respectively given by

$$
\begin{aligned}
& k^{\dagger}=(-1)^{q} k, \\
& k^{\prime \dagger}=(-1)^{q+1} k^{\prime} .
\end{aligned}
$$

Therefore, in the case $(p, q)=(\text { even,even })^{11}, k$ and $k^{\prime}$ are hermitian and anti-hermitian matrices, respectively. On the other hand, in the case $(p, q)=\left(\right.$ odd,odd), $k$ and $k^{\prime}$ are anti-hermitian and hermitian matrices, respectively.

The $S O(p, q)$ generators are constructed by

$$
\sigma^{\mu \nu}=-i \frac{1}{4}\left[\gamma^{\mu}, \gamma^{\nu}\right]
$$

and they satisfy

$$
\begin{aligned}
& \left(k \sigma^{\mu \nu}\right)^{\dagger}=(-1)^{q} k \sigma^{\mu \nu}, \\
& \left(k^{\prime} \sigma^{\mu \nu}\right)^{\dagger}=(-1)^{q+1} k^{\prime} \sigma^{\mu \nu} .
\end{aligned}
$$

Therefore, when $(p, q)=($ even,even $), \gamma^{\mu}$ and $\sigma^{\mu \nu}$ are simultaneously hermitianized only by $k$. Meanwhile, when $(p, q)=($ odd,odd $), \gamma^{\mu}$ and $\sigma^{\mu \nu}$ are simultaneously hermitianized only by $k^{\prime}$.

\footnotetext{
${ }^{10} k^{\prime}$ corresponds to $A$ matrix in Ref.[65] up to a proportional factor.

${ }^{11}$ Remember $p+q$ is even.
} 


\subsection{2 $S O(l, m)$ gamma matrices $(l+m$ : odd $)$ from $S O(p, q)(p+q$ : even $)$}

Next, we consider odd dimensional space-times:

$$
l+m \text { : odd } .
$$

As is well known, $S O(l, m)(l+m$ : odd) gamma matrices can be "constructed" by $S O(p, q)(p+q$ : even) gamma matrices.

- In the case of $S O(l, m)=S O(p+1, q)$

The $S O(p+1, q)$ gamma matrices $\gamma^{a}(a=1,2, \cdots, p+q+1)$ satisfy

$$
\left\{\gamma^{a}, \gamma^{b}\right\}=2 \eta^{a b}
$$

with

$$
\eta^{a b}=\operatorname{diag}(\overbrace{+,+, \cdots,+}^{p}, \overbrace{-,-, \cdots,-}^{q},+) .
$$

$\gamma^{a}$ consist of the $S O(p, q)$ gamma matrices $\gamma^{\mu}(\mu=1,2, \cdots, p+q)$ and

$$
\gamma^{p+q+1} \equiv(i)^{\frac{1}{2}(p-q)} \gamma^{1} \gamma^{2} \cdots \gamma^{p+q}
$$

$\gamma^{p+q+1}$ is a hermitian matrix that satisfies $\left(\gamma^{p+q+1}\right)^{2}=1$. With $k(30), \gamma^{a}=\left(\gamma^{\mu}, \gamma^{p+q+1}\right)$ are hermitianized as

$$
\left(k \gamma^{a}\right)^{\dagger}=k \gamma^{a} .
$$

Notice that unlike $k, k^{\prime}$ does not hermitianize all of the $S O(p+1, q)$ gamma matrices: $k^{\prime} \gamma^{\mu}$ are hermitian (as stated above) but $k^{\prime} \gamma^{p+q+1}$ is anti-hermitian. Then, all of the gamma matrices of $S O(p+1, q)$ can be hermitian multiplied only by $k$. Similarly, it can be shown that the $S O(p+1, q)$ generators

$$
\sigma^{a b}=-i \frac{1}{4}\left[\gamma^{a}, \gamma^{b}\right]
$$

satisfy

$$
\left(k \sigma^{a b}\right)^{\dagger}=(-1)^{q} k \sigma^{a b} .
$$

- In the case of $S O(l, m)=S O(p, q+1)$

The $S O(p, q+1)$ gamma matrices $\gamma^{a}(a=1,2, \cdots, p+q+1)$ satisfy

$$
\left\{\gamma^{a}, \gamma^{b}\right\}=2 \eta^{a b}
$$

with

$$
\eta^{a b}=\operatorname{diag}(\overbrace{+,+, \cdots,+}^{p}, \overbrace{-,-, \cdots,-}^{q},-) .
$$

$\gamma^{a}(a=1,2, \cdots, p+q+1)$ are explicitly given by the $S O(p, q)$ gamma matrices $\gamma^{\mu}(\mu=1,2, \cdots, p+$ $q)$ and

$$
\tilde{\gamma}^{p+q+1} \equiv(i)^{\frac{1}{2}(p-q)+1} \gamma^{1} \gamma^{2} \cdots \gamma^{p+q} .
$$


$\tilde{\gamma}^{p+q+1}$ is an anti-hermitian matrix that satisfies $\left(\tilde{\gamma}^{p+q+1}\right)^{2}=-1$. With $k^{\prime}(31)$, all of $\gamma^{a}=$ $\left(\gamma^{\mu}, \tilde{\gamma}^{p+q+1}\right)$ are hermitianized :

$$
\left(k^{\prime} \gamma^{a}\right)^{\dagger}=k^{\prime} \gamma^{a} .
$$

Notice that $k$ does not hermitianize all of the $S O(p, q+1)$ gamma matrices: $k \gamma^{\mu}$ are hermitian (as stated above) but $k \tilde{\gamma}^{p+q+1}$ is anti-hermitian. Consequently, the gamma matrices of $S O(p, q+1)$ are hermitianized only by $k^{\prime}$. Similarly, $S O(p, q+1)$ generators

$$
\sigma^{a b}=-i \frac{1}{4}\left[\gamma^{a}, \gamma^{b}\right]
$$

satisfy

$$
\left(k^{\prime} \sigma^{a b}\right)^{\dagger}=(-1)^{q+1} k^{\prime} \sigma^{a b} .
$$

\subsubsection{Consistency}

The $S O(l, m)$ gamma matrices $(l+m$ : odd) are constructed either by $S O(l-1, m)$ or $S O(l, m-1)$ gamma matrices by the methods stated above. Then, there exist two superficially different ways for the construction of $S O(l, m)(l+m$ : odd) gamma matrices. We discuss consistency of such two methods.

- $S O(l, m)=S O($ odd, even $)$

The gamma matrices of $S O(l, m)(l, m)=$ (odd, even) constructed from those of $S O(l-1, m)$ become hermitian (40) multiplied by $k$. Since $(p, q)=(l-1, m)=($ even, even), $k$ is hermitian in the present case. Meanwhile, the gamma matrices of $S O(l, m)(l, m)=($ odd, even) constructed from those of $S O(l, m-1)$ are hermitianized (46) by $k^{\prime} . k^{\prime}$ is also hermitian, since $(p, q)=$ $(l, m-1)=($ odd, odd $)$. Thus, in either case, we reach the same conclusion: hermitianization of $S O$ (odd, even) gamma matrices is performed by the hermitian matrix ${ }^{12}$. Consequently, for instance, $S O(1,2), S O(1,4), S O(3,2)$ gamma matrices respectively become hermitian matrices multiplied by their corresponding hermitian matrices.

- $S O(m, l)=S O($ even, odd $)$

Since the overall signs of metrics of $S O(m, l)$ and $S O(l, m)$ are interchanged, their gamma matrices are equal up to the overall imaginary unit. Therefore, their hermitianizing matrices are also equal up to the overall imaginary unit. As discussed above, the $S O$ (odd,even) gamma matrices are hermitianized by a hermitian matrix. Therefore, the hermitianizing matrix of $S O$ (even,odd) gamma matrices is given by an anti-hermitian matrix ${ }^{13}$. For instance, $S O(2,1), S O(4,1), S O(2,3)$ gamma matrices respectively become hermitian multiplied by their corresponding anti-hermitian matrices.

\footnotetext{
${ }^{12}$ The corresponding $S O$ (odd,even) generators also become hermitian matrices multiplied by the same hermitian matrix.

${ }^{13}$ The corresponding $S O$ (even,odd) generators also become anti-hermitian matrices multiplied by the same antihermitian matrix
} 


\subsubsection{Relations between $S O(1,2 p)$ and $S O(2 p+1)$ gamma matrices}

The $S O(1,2 p)$ gamma matrices consist of $S O(0,2 p)$ gamma matrices, $\gamma^{1}, \gamma^{2}, \cdots, \gamma^{2 p}$, and $\gamma^{2 p+1}$ :

$$
\gamma^{2 p+1} \equiv(-i)^{p} \gamma^{1} \gamma^{2} \cdots \gamma^{2 p}
$$

Here, we substituted $(p, q)$ with $(0,2 p)$ in (39) to derive (49). Their hermitianizing matrix is given by $(30)$

$$
k=i^{p(2 p-1)} \gamma^{1} \gamma^{2} \cdots \gamma^{2 p}=(-1)^{p} \gamma^{2 p+1},
$$

which is hermitian. The hermitianized $S O(1,2 p)$ gamma matrices are $k \gamma^{1}, k \gamma^{2}, \cdots, k \gamma^{2 p}, k \gamma^{2 p+1}$ where the last one is proportional to the unit matrix: $k \gamma^{2 p+1}=(-1)^{p} \mathbf{1}$. From anti-commutativity between $k$ and $\gamma^{a}$, i.e. $k \gamma^{a}=-\gamma^{a} k$, we can see that the hermitianized $S O(0,2 p)$ gamma matrices $k \gamma^{1}, k \gamma^{2}, \cdots, k \gamma^{2 p}$ and the matrix $k(50)$ amount to $S O(2 p+1)$ gamma matrices. This unique relation between $S O(1,2 p)$ and $S O(2 p+1)$ underlies relations between the compact and hybrid Hopf maps as we shall discuss in Section 3.1.

\section{Non-compact 1st Hopf Map and Fuzzy Two-Hyperboloid}

In this section, we introduce the non-compact 1st Hopf map (Section 3.1) and the corresponding fuzzy two-hyperboloid $H_{F}^{2,0}$ (Section 3.2), mainly based on Refs.[40, 45] ${ }^{14}$.

\subsection{Non-compact 1st Hopf map: $H^{2,0} \simeq H^{2,1} / S^{1}$}

The 1st non-compact Hopf map is given by

$$
H^{2,1} \stackrel{S^{1}}{\longrightarrow} H^{2,0}
$$

which can be rewritten as the following form more familiar to physicists,

$$
A d S^{3} \stackrel{U(1)}{\longrightarrow} E A d S^{2}
$$

Here, $E A d S^{2}=H^{2,0}$ is a two-leaf hyperboloid whose symmetry group is $S O(1,2)$. The gamma matrices of the $S O(1,2)$ group, $\tau^{i}(i=1,2,3)$, satisfy the following anti-commutation relations

$$
\left\{\tau^{i}, \tau^{j}\right\}=2 \eta^{i j}
$$

with $\eta_{i j}=\operatorname{diag}(-,-,+)$. Note $S O(1,2) \simeq S U(1,1)$, and $\tau^{i}$ are given by the $S U(1,1)$ "Pauli matrices":

$$
\tau^{1}=i \sigma^{2}, \quad \tau^{2}=-i \sigma^{1}, \quad \tau^{3}=\sigma^{3} .
$$

They satisfy the commutation relations

$$
\left[\tau^{i}, \tau^{j}\right]=-2 i \epsilon^{i j k} \tau_{k}
$$

\footnotetext{
${ }^{14}$ The split 1st Hopf map, $H^{2,1} \stackrel{H^{1,0}}{\longrightarrow} H^{1,1}$, cannot be realized by using the usual imaginary unit [45]. To realize the split 1st Hopf map, we need to utilize the split-imaginary unit, and such construction will be discussed in Appendix B.
} 
where $\epsilon^{i j k}$ is 3 rank antisymmetric tensor with $\epsilon^{123}=1$. With use of $S O(0,2)$ gamma matrices $\tau^{1}, \tau^{2}$, the hermitianizing matrix (50) is constructed as

$$
\kappa=i \tau^{1} \tau^{2}=\sigma^{3}
$$

and the hermitianized gamma matrices are

$$
\kappa^{i} \equiv \sigma^{3} \tau^{i}=\left(\sigma^{1}, \sigma^{2}, 1\right) .
$$

With an $S O(1,2)$ Dirac spinor $\phi$ subject to a "normalization" constraint $\phi^{\dagger} \sigma^{3} \phi=1$, the 1 st non-compact Hopf map is given by

$$
\phi \rightarrow x^{i}=\phi^{\dagger} \kappa^{i} \phi
$$

and $x^{i}$ in (58) automatically satisfy the condition of $H^{2,0}$ :

$$
\eta_{i j} x^{i} x^{j}=-x^{2}-y^{2}+z^{2}=\left(\phi^{\dagger} \sigma^{3} \phi\right)^{2}=1,
$$

where $x=x^{1}, y=x^{2}, z=x^{3}$. Thus, $x^{i}(58)$ are regarded as coordinates on two-leaf hyperboloid. In particular, since $z=\phi^{\dagger} \phi \geq 0$, the present construction corresponds to the upper leaf of two-leaf hyperboloid.

Here, we also mention the "derivation" of the hybrid Hopf map from the compact Hopf map. The original 1st Hopf map is given by

$$
\phi \quad \rightarrow \quad x^{i}=\phi^{\dagger} \sigma^{i} \phi
$$

where $\phi$ is subject to $\phi^{\dagger} \phi=1 . x^{i}$ automatically satisfy the condition of $S^{2}$ :

$$
x^{2}+y^{2}+z^{2}=\left(\phi^{\dagger} \phi\right)^{2}=1 .
$$

As mentioned in Section 2.3.4, there is a unique relation between $S O(2 p+1)$ and $S O(1,2 p)$ gamma matrices. In the present case $p=1, \kappa=\sigma^{3}$ and $\left(\kappa^{1}, \kappa^{2}\right)=\left(\sigma^{1}, \sigma^{2}\right)$ amount to the $S O(3)$ gamma matrices. With (60), the compact Hopf map (61) can be restated as

$$
\left(\phi^{\dagger} \kappa^{1} \phi\right)^{2}+\left(\phi^{\dagger} \kappa^{2} \phi\right)^{2}+\left(\phi^{\dagger} \kappa \phi\right)^{2}=\left(\phi^{\dagger} \phi\right)^{2} .
$$

Interchanging the right-hand side and the last term on the left-hand side in (62), we have

$$
-\left(\phi^{\dagger} \kappa^{1} \phi\right)^{2}-\left(\phi^{\dagger} \kappa^{2} \phi\right)^{2}+\left(\phi^{\dagger} \phi\right)^{2}=\left(\phi^{\dagger} \kappa \phi\right)^{2},
$$

which is exactly equal to the relation (59). Thus, one can "derive" the non-compact 1st Hopf map from the compact Hopf map. Similarly, the 2nd and 3rd hybrid Hopf maps can also be obtained by their corresponding compact 2nd and 3rd Hopf maps from the relations of gamma matrices discussed in Section 2.3.4.

\subsection{Fuzzy two-hyperboloid: $H_{F}^{2,0}$}

We argue the Schwinger operator formulation of fuzzy two-hyperboloid with emphasis on its relation to the non-compact 1st Hopf map. 


\subsubsection{Finite dimensional non-unitary representation}

By replacing $\phi$ with the Schwinger operator $\Phi$ in (60), the coordinates on fuzzy two-hyperboloid are constructed as [40]

$$
X^{i}=\Phi^{\dagger} \kappa^{i} \Phi
$$

$X^{i}$ are hermitian operators. For $X^{i}$ to satisfy the $S U(1,1)$ algebra

$$
\left[X^{i}, X^{j}\right]=-2 i \epsilon^{i j k} X_{k}
$$

the components of Schwinger operator should satisfy generalized creation and annihilation relations:

$$
\left[\Phi_{\alpha}, \Phi_{\beta}^{\dagger}\right]=\left(\sigma^{3}\right)_{\alpha \beta}, \quad\left[\Phi_{\alpha}, \Phi_{\beta}\right]=\left[\Phi_{\alpha}^{\dagger}, \Phi_{\beta}^{\dagger}\right]=0 .
$$

Note that $\Phi_{2}$ and $\Phi_{2}^{\dagger}$ obey an unusual commutation relation, $\left[\Phi_{2}, \Phi_{2}^{\dagger}\right]=-1$. From (64) and (66), square of the radius of fuzzy two-hyperboloid is derived as

$$
\eta_{i j} X^{i} X^{j}=-X^{2}-Y^{2}+Z^{2}=\left(\Phi^{\dagger} \sigma^{3} \Phi\right)\left(\Phi^{\dagger} \sigma^{3} \Phi+2\right) .
$$

The eigenvalues of (67) are given by

$$
\eta_{i j} X^{i} X^{j}=n(n+2)
$$

with

$$
\Phi^{\dagger} \sigma^{3} \Phi=n_{1}+n_{2} \equiv n,
$$

( $n_{1}$ and $n_{2}$ are non-negative integers) and the eigenstates are

$$
\left|n_{1}, n_{2}\right\rangle=\frac{1}{\sqrt{n_{1} ! n_{2} !}}\left(\Phi_{1}^{\dagger}\right)^{n_{1}}\left(\Phi_{2}^{\dagger}\right)^{n_{2}}|0\rangle,
$$

with $|0\rangle$ that satisfies $\Phi_{1}|0\rangle=\Phi_{2}|0\rangle=0$. Here, we used that

$$
\begin{aligned}
& \Phi_{1}^{\dagger} \Phi_{1}\left|n_{1}, n_{2}\right\rangle=n_{1}\left|n_{1}, n_{2}\right\rangle, \\
& \Phi_{2}^{\dagger} \Phi_{2}\left|n_{1}, n_{2}\right\rangle=-n_{2}\left|n_{1}, n_{2}\right\rangle .
\end{aligned}
$$

Note the minus sign in front of the right-hand side of (71b): Physically, such minus sign indicates that $|0\rangle$ is an "unstable" vacuum for the 2 nd oscillator mode, since $|0\rangle$ corresponds to the highest "energy" state. From (71), one finds that $X^{3}$ takes the eigenvalues

$$
X^{3}=\Phi_{1}^{\dagger} \Phi_{1}+\Phi_{2}^{\dagger} \Phi_{2}=n_{1}-n_{2}=n, n-2, n-4, \cdots,-n .
$$

These spectra are same as those of fuzzy two-sphere.

Dual state of (70)

$$
\left\langle n_{1}, n_{2}\right|=\frac{1}{\sqrt{n_{1} ! n_{2} !}}\langle 0| \Phi_{1}^{n_{1}} \Phi_{2}^{n_{2}},
$$

yields negative norm for odd $n_{2}$ : $\left\langle n_{1}, n_{2} \mid n_{1}^{\prime}, n_{2}^{\prime}\right\rangle=(-1)^{n_{2}} \delta_{n_{1} n_{1}^{\prime}} \delta_{n_{2} n_{2}^{\prime}}$. More suitable dual state may be given by

$$
\left\langle\left\langle n_{1}, n_{2}\right|=\frac{1}{\sqrt{n_{1} ! n_{2} !}}\langle 0|\left(\Phi^{1}\right)^{n_{1}}\left(\Phi^{2}\right)^{n_{2}}=(-1)^{n_{2}}\left\langle n_{1}, n_{2}\right|,\right.
$$


where $\Phi^{\alpha} \equiv\left(\sigma^{3}\right)^{\alpha \beta} \Phi_{\beta}$, i.e. $\left(\Phi^{1}, \Phi^{2}\right)=\left(\Phi_{1},-\Phi_{2}\right)$. (74) satisfies $\left\langle\left\langle n_{1}, n_{2} \mid n_{1}^{\prime}, n_{2}^{\prime}\right\rangle=\delta_{n_{1} n_{1}^{\prime}} \delta_{n_{2} n_{2}^{\prime}}\right.$.

Such non-unitary construction of fuzzy hyperboloid is a straightforward generalization of that of fuzzy sphere, and readily applicable to represent states on fuzzy hyperboloids in arbitrary higher dimensions [44]. However, such non-hermitian construction is rather counterintuitive, since in the large $n$ limit, the spectra (72) do not reduce to the values of the 3rd coordinate of the classical upper leaf (or lower-leaf) hyperboloid, i.e. $x^{3} \geq n$ (or $x^{3} \leq-n$ ).

\subsubsection{Infinite dimensional unitary representation}

The above mentioned "problem" of the non-unitary construction of fuzzy hyperboloid is amended by adopting infinite dimensional unitary representation. Unitary representation can readily be obtained by a redefinition of the creation and annihilation Schwinger operators. We interchange $\Phi_{2}$ and $\Phi_{2}^{\dagger}$ for the components of the Schwinger operator to satisfy the usual commutation relations:

$$
\left[\Phi_{\alpha}, \Phi_{\beta}^{\dagger}\right]=\delta_{\alpha \beta}, \quad\left[\Phi_{\alpha}, \Phi_{\beta}\right]=\left[\Phi_{\alpha}^{\dagger}, \Phi_{\beta}^{\dagger}\right]=0
$$

With the new Schwinger operators, $X^{i}(64)$ are represented as

$$
\begin{aligned}
& X^{1}=\Phi_{1}^{\dagger} \Phi_{2}^{\dagger}+\Phi_{2} \Phi_{1}, \\
& X^{2}=-i \Phi_{1}^{\dagger} \Phi_{2}^{\dagger}+i \Phi_{2} \Phi_{1}, \\
& X^{3}=\Phi_{1}^{\dagger} \Phi_{1}+\Phi_{2} \Phi_{2}^{\dagger}=\Phi_{1}^{\dagger} \Phi_{1}+\Phi_{2}^{\dagger} \Phi_{2}+1 .
\end{aligned}
$$

These are still hermitian operators, but $X^{1}$ and $X^{2}$ (that are originally constructed by antihermitian gamma matrices) become particle-number non-conserving operators. One may readily check that $(76)$ indeed satisfy the $S U(1,1)$ commutation relations under (75). The radius of the fuzzy hyperboloid (67) is now written as

$$
\eta_{i j} X^{i} X^{j}=\left(\Phi^{\dagger} \sigma^{3} \Phi-1\right)\left(\Phi^{\dagger} \sigma^{3} \Phi+1\right),
$$

and the simultaneous eigenstates of (77) and (76c) are

$$
\left.\mid n_{1}, n_{2}\right)=\frac{1}{\sqrt{n_{1} ! n_{2} !}}\left(\Phi_{1}^{\dagger}\right)^{n_{1}}\left(\Phi_{2}^{\dagger}\right)^{n_{2}}|\mathrm{vac}\rangle
$$

with non-negative integers, $n_{1}, n_{2}$, and eigenvalues

$$
\begin{aligned}
& \eta_{i j} X^{i} X^{j}=\left(n_{1}-n_{2}\right)^{2}-1, \\
& X^{3}=n_{1}+n_{2}+1 .
\end{aligned}
$$

Here, $|v a c\rangle$ is the true vacuum of the newly defined Schwinger operator $\Phi_{1,2}$ :

$$
\Phi_{1}|\mathrm{vac}\rangle=\Phi_{2}|\mathrm{vac}\rangle=0
$$

and the dual state of (78) is obtained as

$$
\left(n_{1}, n_{2} \mid=\frac{1}{\sqrt{n_{1} ! n_{2} !}}\langle\operatorname{vac}|\left(\Phi_{1}\right)^{n_{1}}\left(\Phi_{2}\right)^{n_{2}},\right.
$$


which always yields positive norm: $\left(n_{1}, n_{2} \mid n_{1}^{\prime}, n_{2}^{\prime}\right)=\delta_{n_{1} n_{1}^{\prime}} \delta_{n_{2} n_{2}^{\prime}}$.

Since $\eta_{i j} X^{i} X^{j}$ indicates square of the radius of fuzzy hyperboloid, it should have positive eigenvalues. By the interchange symmetry between $\Phi_{1}$ and $\Phi_{2}$ in (76), we can take $n_{1} \geq n_{2}$ in (78) without loss of generality. Instead of $n_{1}$ and $n_{2}$, we introduce new parameters $n$ and $l$,

$$
\begin{aligned}
& n \equiv n_{1}-n_{2} \geq 0, \\
& l \equiv n_{2} \geq 0 .
\end{aligned}
$$

The spectra of (79) are rewritten as

$$
\begin{aligned}
& \eta_{i j} X^{i} X^{j}=n^{2}-1, \\
& X^{3}=n+1+2 l=n+1, n+3, n+5, n+7, \cdots .
\end{aligned}
$$

Here, $n$ and $l$ (82) indicate the radius of hyperboloid and the 3rd coordinate on the upper hyperboloid, respectively. In the large $n$ limit, the radius of fuzzy hyperboloid behaves as $\sim n$ and $X^{3}$ is $\geq n$. Thus in unitary representation, the spectra of fuzzy hyperboloid naturally reduce to (the upper leaf of) the classical two-leaf hyperboloid. For the (semi-)positivity of square of the radius in (83a), $n$ should be taken as $n \geq 1$. With $n$ and $l,(78)$ is represented as

$$
\mid n, l)=\frac{1}{\sqrt{(n+l) ! l !}}\left(\Phi_{1}^{\dagger}\right)^{n+l}\left(\Phi_{2}^{\dagger}\right)^{l}|\mathrm{vac}\rangle
$$

which describes a state at $X^{3}=n+1+2 l(l=0,1,2,3, \cdots)$ on the upper leaf of the fuzzyhyperboloid. In the language of the $S U(1,1)$ representation theory, the present unitary representation corresponds to the discrete series $[66,67]^{15}$.

We have two kinds of two-dimensional hyperboloids, two-leaf hyperboloid $H^{2,0}$ and one-leaf hyperboloid $H^{1,1}\left(A d S^{2}\right)$. Either coordinates of their corresponding fuzzy manifolds satisfy the $S U(1,1)$ algebra. Their difference is specified by choosing different unitary irreducible representations of $S U(1,1)$. For fuzzy $H^{1,1}$, one has to adopt the principal series [31, 42], while for fuzzy $H^{2,0}$ one has to adopt the discrete series. Thus, choice of unitary irreducible representation is crucial in description of fuzzy manifold. It should also be mentioned that such distinction is lacking in the non-unitary construction of fuzzy hyperboloid.

The relevant mathematical structures of 2D fuzzy sphere and fuzzy hyperboloids are summarized in Table 1.

\section{Non-compact 2nd Hopf Maps and Fuzzy Four-Hyperboloids}

In this section, we argue a construction of four-dimensional fuzzy hyperboloids based on noncompact 2nd Hopf maps. First, we introduce two types of non-compact 2nd Hopf maps, the split

\footnotetext{
${ }^{15}$ Detail correspondences to the $S U(1,1)$ representation theory in Refs. [66, 67] are as follows. In the discrete series, the eigenvalues of the $S U(1,1)$ Casimir are given by $\eta_{i j} X^{i} X^{j}=4 j(j-1)$ with $j=1,3 / 2,2, \cdots$ ( $j$ corresponds to $k$ in Refs.[66, 67]), and those of $\frac{1}{2} X^{3}$ are $\mu=j, j+1, j+2, j+3, \cdots$. The identification to the notation of the present paper is $j=\frac{1}{2}(n+1)$ and $\mu=\frac{1}{2}(n+1)+l(l=0,1,2, \cdots)$. Such identification exactly reproduces (83) from the known formulas of the $S U(1,1)$ representation theory.
} 


\begin{tabular}{|c||c|c|c|c|c|}
\hline Symmetry & Original manifold & Hopf Map & $k$ matrix & Enhanced Algebra & Fuzzy Manifold \\
\hline \hline$S O(3)$ & $S^{2}=H^{0,2}$ & Compact & Hermite & $S O(3) \simeq S U(2)$ & $S_{F}^{2} \simeq \mathbb{C} P^{1,0}$ \\
\hline$S O(2,1)$ & $d S^{2}=A d S^{2}=H^{1,1}$ & Split & Anti-hermite & $S O(1,2) \simeq S U(1,1)$ & $H_{F}^{1,1} \simeq \mathbb{C}^{\prime} P^{0,1}$ \\
\hline$S O(1,2)$ & $E A d S^{2}=H^{2,0}$ & Hybrid & Hermite & $S O(2,1) \simeq S U(1,1)$ & $H_{F}^{2,0} \simeq \mathbb{C} P^{0,1}$ \\
\hline
\end{tabular}

Table 1: Fuzzyfication of two-hyperboloids and related properties. See Appendix $\mathrm{C}$ for $\mathbb{C}^{p, q}$ and $\mathbb{C}^{\prime} P^{p, q}$.

and hybrid types (Section 4.1 and 4.3). Corresponding to two types of non-compact Hopf maps, we construct fuzzy $H^{2,2}$ and fuzzy $H^{4,0}$ (Section 4.2 and 4.4). Geometrical structures of the fuzzy four-hyperboloids are also discussed (Section 4.5).

\subsection{Split 2nd Hopf map: $H^{2,2} \simeq H^{4,3} / H^{2,1}$}

In the explicit realization of the split Hopf map [45], the hermitianizing matrix for $S O(3,2)$ gamma matrices plays a crucial role. The $S O(3,2)$ gamma matrices $\gamma^{a}(a=1,2,3,4,5)$ satisfy

$$
\left\{\gamma^{a}, \gamma^{b}\right\}=2 \eta^{a b}
$$

with $\eta^{a b}=\eta_{a b}=\left(\eta_{i j},+,+\right)=\operatorname{diag}(-,-,+,+,+)$. They are explicitly given by

$$
\gamma^{i}=-\tau^{i} \otimes \sigma^{2}, \quad \gamma^{4}=1 \otimes \sigma^{1}, \quad \gamma^{5}=\gamma^{1} \gamma^{2} \gamma^{3} \gamma^{4}=1 \otimes \sigma^{3},
$$

or

$$
\gamma^{i}=\left(\begin{array}{cc}
0 & i \tau^{i} \\
-i \tau^{i} & 0
\end{array}\right), \quad \gamma^{4}=\left(\begin{array}{ll}
0 & 1 \\
1 & 0
\end{array}\right), \quad \gamma^{5}=\left(\begin{array}{cc}
1 & 0 \\
0 & -1
\end{array}\right)
$$

where $\tau^{i}(i=1,2,3)$ are the $S U(1,1)$ Pauli matrices (54). $\gamma^{1}$ and $\gamma^{2}$ are anti-hermitian while $\gamma^{3}$, $\gamma^{4}$ and $\gamma^{5}$ are hermitian. By the formula $(30), S O(3,2)$ hermitianizing matrix is constructed as

$$
k=i \gamma^{4} \gamma^{3}=\left(\begin{array}{cc}
\sigma^{3} & 0 \\
0 & \sigma^{3}
\end{array}\right),
$$

and hermitianized "gamma" matrices are given by

$$
k^{a}=k \gamma^{a},
$$

or

$$
\begin{array}{rlrl}
k^{1} & =\left(\begin{array}{cc}
0 & i \sigma^{1} \\
-i \sigma^{1} & 0
\end{array}\right), & k^{2}=\left(\begin{array}{cc}
0 & i \sigma^{2} \\
-i \sigma^{2} & 0
\end{array}\right), \quad k^{3}=\left(\begin{array}{cc}
0 & i 1_{2} \\
-i 1_{2} & 0
\end{array}\right), \\
k^{4}=\left(\begin{array}{cc}
0 & \sigma^{3} \\
\sigma^{3} & 0
\end{array}\right), & k^{5}=\left(\begin{array}{cc}
\sigma^{3} & 0 \\
0 & -\sigma^{3}
\end{array}\right) .
\end{array}
$$


Coordinates on $H^{4,3}$ are represented by a "normalized" $S O(3,2)$ Dirac spinor $\psi$ (the $S O(3,2)$ Hopf spinor) subject to the constraint

$$
\psi^{\dagger} k \psi=\psi_{1}^{*} \psi_{1}-\psi_{2}^{*} \psi_{2}+\psi_{3}^{*} \psi_{3}-\psi_{4}^{*} \psi_{4}=1
$$

With such $\psi$, the 2 nd split Hopf map, $H^{4,3} \stackrel{H^{2,1}}{\longrightarrow} H^{2,2}$, is realized as

$$
\psi \quad \rightarrow \quad x^{a}=\psi^{\dagger} k^{a} \psi
$$

It is easily checked that $x^{a}$ satisfy the condition of $H^{2,2}$ :

$$
\eta_{a b} x^{a} x^{b}=-x^{1} x^{1}-x^{2} x^{2}+x^{3} x^{3}+x^{4} x^{4}+x^{5} x^{5}=\left(\psi^{\dagger} k \psi\right)^{2}=1 .
$$

Commutators of $\gamma^{a}$ provide the $S O(3,2)$ generators

$$
\gamma^{a b}=-i \frac{1}{4}\left[\gamma^{a}, \gamma^{b}\right]
$$

or

$$
\begin{aligned}
& \gamma^{i j}=-\frac{1}{2} \epsilon^{i j k}\left(\begin{array}{cc}
\tau_{k} & 0 \\
0 & \tau_{k}
\end{array}\right), \quad \gamma^{i 4}=\frac{1}{2}\left(\begin{array}{cc}
\tau^{i} & 0 \\
0 & -\tau^{i}
\end{array}\right), \\
& \gamma^{i 5}=-\frac{1}{2}\left(\begin{array}{cc}
0 & \tau^{i} \\
\tau^{i} & 0
\end{array}\right), \quad \gamma^{45}=\frac{i}{2}\left(\begin{array}{cc}
0 & 1 \\
-1 & 0
\end{array}\right),
\end{aligned}
$$

and they satisfy

$$
\left[\gamma_{a b}, \gamma_{c d}\right]=i\left(\eta_{a c} \gamma_{b d}-\eta_{a d} \gamma_{b c}+\eta_{b d} \gamma_{a c}-\eta_{b c} \gamma_{a d}\right) .
$$

As easily checked, $k \gamma^{a b}$ are hermitian as well.

\subsection{Fuzzy split four-hyperboloid: $H_{F}^{2,2}$}

By replacing the $S O(3,2)$ Hopf spinor with a four-component Schwinger operator, we construct fuzzy four-hyperboloid $H_{F}^{2,2}$.

\subsubsection{Finite dimensional unitary representation}

As a natural generalization of the $S U(1,1)$ Schwinger operator, we introduce the $S O(3,2)$ Schwinger operator $\Phi=\left(\Phi_{1}, \Phi_{2}, \Phi_{3}, \Phi_{4}\right)^{t}$ whose components satisfy

$$
\left[\Phi_{\alpha}, \Phi_{\beta}^{\dagger}\right]=(k)_{\alpha \beta}, \quad\left[\Phi_{\alpha}, \Phi_{\beta}\right]=\left[\Phi_{\alpha}^{\dagger}, \Phi_{\beta}^{\dagger}\right]=0
$$

where $k$ is the $S O(3,2)$ hermitianizing matrix $(88)$. With $\Phi$, coordinates on $H_{F}^{2,2}$ are constructed as

$$
X^{a}=\Phi^{\dagger} k^{a} \Phi
$$

Square of the radius and the 5 th coordinate of $H_{F}^{2,2}$ are explicitly given by

$$
\begin{aligned}
& \eta_{a b} X^{a} X^{b}=-X^{1} X^{1}-X^{2} X^{2}+X^{3} X^{3}+X^{4} X^{4}+X^{5} X^{5}=\left(\Phi^{\dagger} k \Phi\right)\left(\Phi^{\dagger} k \Phi+4\right), \\
& X^{5}=\Phi_{1}^{\dagger} \Phi_{1}-\Phi_{2}^{\dagger} \Phi_{2}-\Phi_{3}^{\dagger} \Phi_{3}+\Phi_{4}^{\dagger} \Phi_{4} .
\end{aligned}
$$


Their simultaneous eigenstates are

$$
\left|n_{1}, n_{2}, n_{3}, n_{4}\right\rangle=\frac{1}{\sqrt{n_{1} ! n_{2} ! n_{3} ! n_{4} !}}\left(\Phi_{1}^{\dagger}\right)^{n_{1}}\left(\Phi_{2}^{\dagger}\right)^{n_{2}}\left(\Phi_{3}^{\dagger}\right)^{n_{3}}\left(\Phi_{4}^{\dagger}\right)^{n_{4}}|0\rangle
$$

where $n_{1}, n_{2}, n_{3}, n_{4}$ are non-negative integers. Furthermore, $\left|n_{1}, n_{2}, n_{3}, n_{4}\right\rangle$ are simultaneous eigenstates of four number operators made of the Schwinger operator:

$$
\begin{aligned}
& \Phi_{1}^{\dagger} \Phi_{1}\left|n_{1}, n_{2}, n_{3}, n_{4}\right\rangle=n_{1}\left|n_{1}, n_{2}, n_{3}, n_{4}\right\rangle \\
& \Phi_{2}^{\dagger} \Phi_{2}\left|n_{1}, n_{2}, n_{3}, n_{4}\right\rangle=-n_{2}\left|n_{1}, n_{2}, n_{3}, n_{4}\right\rangle \\
& \Phi_{3}^{\dagger} \Phi_{3}\left|n_{1}, n_{2}, n_{3}, n_{4}\right\rangle=n_{3}\left|n_{1}, n_{2}, n_{3}, n_{4}\right\rangle \\
& \Phi_{4}^{\dagger} \Phi_{4}\left|n_{1}, n_{2}, n_{3}, n_{4}\right\rangle=-n_{4}\left|n_{1}, n_{2}, n_{3}, n_{4}\right\rangle .
\end{aligned}
$$

Hence, the eigenvalues of (99) are derived as

$$
\begin{aligned}
& \eta_{a b} X^{a} X^{b}=n(n+4), \\
& X^{5}=n_{1}+n_{2}-n_{3}-n_{4},
\end{aligned}
$$

where

$$
n \equiv n_{1}+n_{2}+n_{3}+n_{4}
$$

With a given $n$, square of the radius of $H_{F}^{2,2}$ is fixed, and $X^{5}$ takes the following values:

$$
X^{5}=n, n-2, n-4, n-6, \cdots,-n .
$$

The spectra (102) coincide with those of the fuzzy four-sphere. The eigenstates (100) are still degenerate for given $n$ and $X^{5}$, and such "internal structure" gives rise to symmetry enhancement of fuzzy ultra-hyperboloids as we shall see later.

The dual state of (100) for positive inner product is given by

$$
\begin{aligned}
\left\langle n_{1}, n_{2}, n_{3}, n_{4}\right| & =\frac{1}{\sqrt{n_{1} ! n_{2} ! n_{3} ! n_{4} !}}\langle 0|\left(\Phi^{4}\right)^{n_{4}}\left(\Phi^{3}\right)^{n_{3}}\left(\Phi^{2}\right)^{n_{2}}\left(\Phi^{1}\right)^{n_{4}} \\
& =(-1)^{n_{2}+n_{4}} \frac{1}{\sqrt{n_{1} ! n_{2} ! n_{3} ! n_{4} !}}\langle 0|\left(\Phi_{4}\right)^{n_{4}}\left(\Phi_{3}\right)^{n_{3}}\left(\Phi_{2}\right)^{n_{2}}\left(\Phi_{1}\right)^{n_{4}},
\end{aligned}
$$

where $\Phi^{\alpha}=(k)^{\alpha \beta} \Phi_{\beta}$, i.e. $\left(\Phi^{1}, \Phi^{2}, \Phi^{3}, \Phi^{4}\right)=\left(\Phi_{1},-\Phi_{2}, \Phi_{3},-\Phi_{4}\right)$.

\subsubsection{Infinite dimensional unitary representation}

We redefine the Schwinger operator by the interchange, $\Phi_{2} \leftrightarrow \Phi_{2}^{\dagger}$ and $\Phi_{4} \leftrightarrow \Phi_{4}^{\dagger}$, for its components to satisfy the usual commutation relations:

$$
\left[\Phi_{\alpha}, \Phi_{\beta}^{\dagger}\right]=\delta_{\alpha \beta}, \quad\left[\Phi_{\alpha}, \Phi_{\beta}\right]=\left[\Phi_{\alpha}^{\dagger}, \Phi_{\beta}^{\dagger}\right]=0 .
$$


With such newly defined Schwinger operators, $X^{a}(98)$ are rewritten as

$$
\begin{aligned}
& X^{1}=i \Phi_{1}^{\dagger} \Phi_{4}^{\dagger}+i \Phi_{2} \Phi_{3}-i \Phi_{1} \Phi_{4}-i \Phi_{2}^{\dagger} \Phi_{3}^{\dagger}, \\
& X^{2}=\Phi_{1}^{\dagger} \Phi_{4}^{\dagger}-\Phi_{2} \Phi_{3}+\Phi_{1} \Phi_{4}-\Phi_{2}^{\dagger} \Phi_{3}^{\dagger}, \\
& X^{3}=i \Phi_{1}^{\dagger} \Phi_{3}+i \Phi_{4}^{\dagger} \Phi_{2}-i \Phi_{3}^{\dagger} \Phi_{1}-i \Phi_{2}^{\dagger} \Phi_{4}, \\
& X^{4}=\Phi_{1}^{\dagger} \Phi_{3}-\Phi_{4}^{\dagger} \Phi_{2}+\Phi_{3}^{\dagger} \Phi_{1}-\Phi_{2}^{\dagger} \Phi_{4}, \\
& X^{5}=\Phi_{1}^{\dagger} \Phi_{1}-\Phi_{2} \Phi_{2}^{\dagger}-\Phi_{3}^{\dagger} \Phi_{3}+\Phi_{4} \Phi_{4}^{\dagger}=\Phi_{1}^{\dagger} \Phi_{1}-\Phi_{2}^{\dagger} \Phi_{2}-\Phi_{3}^{\dagger} \Phi_{3}+\Phi_{4}^{\dagger} \Phi_{4} .
\end{aligned}
$$

Notice that both $X^{1}$ and $X^{2}$ originally constructed by anti-hermitian gamma matrices become particle-number non-conserving operators, and the others, $X^{3}, X^{4}$ and $X^{5}$, are particle-number conserving operators. From (107), a straightforward calculation shows

$$
\eta_{a b} X^{a} X^{b}=\left(\Phi^{\dagger} k \Phi-2\right)\left(\Phi^{\dagger} k \Phi+2\right) .
$$

The simultaneous eigenstates of $X^{5}(107)$ and (108) are given by ${ }^{16}$

$$
\left.\mid n_{1}, n_{2}, n_{3}, n_{4}\right)=\frac{1}{\sqrt{n_{1} ! n_{2} ! n_{3} ! n_{4} !}}\left(\Phi_{1}^{\dagger}\right)^{n_{1}}\left(\Phi_{2}^{\dagger}\right)^{n_{2}}\left(\Phi_{3}^{\dagger}\right)^{n_{3}}\left(\Phi_{4}^{\dagger}\right)^{n_{4}}|\mathrm{vac}\rangle
$$

with the eigenvalues

$$
\begin{aligned}
& \eta_{a b} X^{a} X^{b}=(n-2)(n+2)=n^{2}-4, \\
& X^{5}=n_{1}-n_{2}+n_{3}-n_{4},
\end{aligned}
$$

where $n$ denotes the eigenvalues of $\Phi^{\dagger} k \Phi$ :

$$
n \equiv n_{1}-n_{2}-n_{3}+n_{4} .
$$

For the semi-positive definiteness of square of the radius, $n$ in (110a) should be taken as $n \geq 2$. With fixed $n, X^{5}$ is given by

$$
X^{5}=n+2\left(n_{3}-n_{4}\right)=n+2 \Delta n,
$$

where $\Delta n \equiv n_{3}-n_{4}=0, \pm 1, \pm 2, \pm 3, \cdots$. Since $\Delta n$ takes an arbitrary integer, the spectra of $X^{5}$ range from $-\infty$ to $+\infty$ with interval 2 .

The dual state of (109) is given by

$$
\left(n_{1}, n_{2}, n_{3}, n_{4} \mid=\frac{1}{\sqrt{n_{1} ! n_{2} ! n_{3} ! n_{4} !}}\langle\operatorname{vac}|\left(\Phi_{4}\right)^{n_{4}}\left(\Phi_{3}\right)^{n_{3}}\left(\Phi_{2}\right)^{n_{2}}\left(\Phi_{1}\right)^{n_{1}},\right.
$$

which gives rise to positive norm:

$$
\left(n_{1}, n_{2}, n_{3}, n_{4} \mid n_{1}^{\prime}, n_{2}^{\prime}, n_{3}^{\prime}, n_{4}^{\prime}\right)=\delta_{n_{1} n_{1}^{\prime}} \delta_{n_{2} n_{2}^{\prime}} \delta_{n_{3} n_{3}^{\prime}} \delta_{n_{4} n_{4}^{\prime}} .
$$

\footnotetext{
${ }^{16}$ Details about the irreducible representation of the $S O(3,2)$ groups are found in Refs.[68, 69].
} 


\subsection{Hybrid 2nd Hopf map: $H^{4,0} \simeq H^{4,3} / S^{3}$}

Next, we construct the hybrid 2nd Hopf map. As suggested in Section 2.2, the $S O(1,4)$ gamma matrices are crucial in constructing the hybrid 2nd Hopf map. The $S O(1,4)$ gamma matrices are introduced so as to satisfy

$$
\left\{\gamma^{a}, \gamma^{b}\right\}=2 \eta^{a b}
$$

with $\eta^{a b}=\eta_{a b}=\operatorname{diag}(-,-,-,-,+)$. Explicitly, the $S O(1,4)$ gamma matrices are given by

$$
\begin{array}{ll}
\gamma^{1}=\left(\begin{array}{cc}
0 & i \sigma^{1} \\
i \sigma^{1} & 0
\end{array}\right), \quad \gamma^{2}=\left(\begin{array}{cc}
0 & i \sigma^{2} \\
i \sigma^{2} & 0
\end{array}\right), \quad \gamma^{3}=\left(\begin{array}{cc}
0 & i \sigma^{3} \\
i \sigma^{3} & 0
\end{array}\right), \\
\gamma^{4}=\left(\begin{array}{cc}
0 & 1_{2} \\
-1_{2} & 0
\end{array}\right), \quad \gamma^{5}=\left(\begin{array}{cc}
1_{2} & 0 \\
0 & -1_{2}
\end{array}\right),
\end{array}
$$

From $(50)$, the $S O(1,4)$ hermitianizing matrix is constructed as

$$
k=-\gamma^{1} \gamma^{2} \gamma^{3} \gamma^{4}=\left(\begin{array}{cc}
1_{2} & 0 \\
0 & -1_{2}
\end{array}\right)=\gamma^{5}
$$

Notice that $S O(1,4)$ and $S O(3,2)$ hermitianizing matrices, (117) and (88), are unitary equivalent. The hermitianized $S O(1,4)$ gamma matrices $k^{a}=k \gamma^{a}$ are derived as

$$
\begin{aligned}
& k^{1}=\left(\begin{array}{cc}
0 & i \sigma^{1} \\
-i \sigma^{1} & 0
\end{array}\right), \quad k^{2}=\left(\begin{array}{cc}
0 & i \sigma^{2} \\
-i \sigma^{2} & 0
\end{array}\right), \quad k^{3}=\left(\begin{array}{cc}
0 & i \sigma^{3} \\
-i \sigma^{3} & 0
\end{array}\right), \\
& k^{4}=\left(\begin{array}{cc}
0 & 1_{2} \\
1_{2} & 0
\end{array}\right), \quad k^{5}=\left(\begin{array}{cc}
1_{2} & 0 \\
0 & 1_{2}
\end{array}\right) .
\end{aligned}
$$

With $k$, we introduce an $S O(1,4)$ Dirac spinor $\psi$ subject to the "normalization" condition

$$
\psi^{\dagger} k \psi=\psi_{1}^{*} \psi_{1}+\psi_{2}^{*} \psi_{2}-\psi_{3}^{*} \psi_{3}-\psi_{4}^{*} \psi_{4}=1
$$

which geometrically represents $H^{4,3}$. Since the hermitianizing matrices of $S O(1,4)$ and $S O(3,2)$ are equivalent, the total manifolds of the split and hybrid 2nd Hopf maps are identically given by $H^{4,3}$. With $\psi$, the hybrid Hopf map is realized as

$$
x^{a}=\psi^{\dagger} k^{a} \psi
$$

or

$$
\begin{aligned}
& x^{1}=i \psi_{1}^{*} \psi_{4}+i \psi_{2}^{*} \psi_{3}-i \psi_{3}^{*} \psi_{2}-i \psi_{4}^{*} \psi_{1}, \\
& x^{2}=\psi_{1}^{*} \psi_{4}-\psi_{2}^{*} \psi_{3}-\psi_{3}^{*} \psi_{2}+\psi_{4}^{*} \psi_{1}, \\
& x^{3}=i \psi_{1}^{*} \psi_{3}-i \psi_{2}^{*} \psi_{4}-i \psi_{3}^{*} \psi_{1}+i \psi_{4}^{*} \psi_{2}, \\
& x^{4}=\psi_{1}^{*} \psi_{3}+\psi_{2}^{*} \psi_{4}+\psi_{3}^{*} \psi_{1}+\psi_{4}^{*} \psi_{2}, \\
& x^{5}=\psi_{1}^{*} \psi_{1}+\psi_{2}^{*} \psi_{2}+\psi_{3}^{*} \psi_{3}+\psi_{4}^{*} \psi_{4},
\end{aligned}
$$

which automatically satisfy the condition of two-leaf four-hyperboloid $H^{4,0}$ :

$$
\eta_{a b} x^{a} x^{b}=-x^{1} x^{1}-x^{2} x^{2}-x^{3} x^{3}-x^{4} x^{4}+x^{5} x^{5}=\left(\psi^{\dagger} k \psi\right)^{2}=1 .
$$

From (121), we find that $x^{5} \geq 0$ and $x^{a}(121)$ are coordinates on the upper leaf of $H^{4,0}$. 


\subsection{Fuzzy two-leaf four-hyperboloid: $H_{F}^{4,0}=E A d S_{F}^{4}$}

We explore the fuzzy version of $H^{4,0}$.

\subsubsection{Finite dimensional non-unitary representation}

We introduce $S O(1,4)$ Schwinger operator $\Phi=\left(\Phi_{1}, \Phi_{2}, \Phi_{3}, \Phi_{4}\right)^{t}$ whose components satisfy

$$
\left[\Phi_{\alpha}, \Phi_{\beta}^{\dagger}\right]=(k)_{\alpha \beta}, \quad\left[\Phi_{\alpha}, \Phi_{\beta}\right]=\left[\Phi_{\alpha}^{\dagger}, \Phi_{\beta}^{\dagger}\right]=0
$$

where $k$ is the $S O(1,4)$ hermitianizing matrix $(117)$. Coordinates on $H_{F}^{4,0}$ are constructed as

$$
X^{a}=\Phi^{\dagger} k^{a} \Phi
$$

which provide

$$
\begin{aligned}
& \eta_{a b} X^{a} X^{b}=-X^{1} X^{1}-X^{2} X^{2}-X^{3} X^{3}-X^{4} X^{4}+X^{5} X^{5}=\left(\Phi^{\dagger} k \Phi\right)\left(\Phi^{\dagger} k \Phi+4\right), \\
& X^{5}=\Phi_{1}^{\dagger} \Phi_{1}+\Phi_{2}^{\dagger} \Phi_{2}+\Phi_{3}^{\dagger} \Phi_{3}+\Phi_{4}^{\dagger} \Phi_{4} .
\end{aligned}
$$

Their simultaneous eigenstates are given by

$$
\left|n_{1}, n_{2}, n_{3}, n_{4}\right\rangle=\frac{1}{\sqrt{n_{1} ! n_{2} ! n_{3} ! n_{4} !}}\left(\Phi_{1}^{\dagger}\right)^{n_{1}}\left(\Phi_{2}^{\dagger}\right)^{n_{2}}\left(\Phi_{3}^{\dagger}\right)^{n_{3}}\left(\Phi_{4}^{\dagger}\right)^{n_{4}}|0\rangle
$$

with the eigenvalues

$$
\begin{aligned}
& \eta_{a b} X^{a} X^{b}=n(n+4), \\
& X^{5}=n_{1}+n_{2}-n_{3}-n_{4} .
\end{aligned}
$$

Here, $n \equiv n_{1}+n_{2}+n_{3}+n_{4}$. (126) is also a simultaneous eigenstate of the four number operators made of the Schwinger operator:

$$
\begin{aligned}
& \Phi_{1}^{\dagger} \Phi_{1}\left|n_{1}, n_{2}, n_{3}, n_{4}\right\rangle=n_{1}\left|n_{1}, n_{2}, n_{3}, n_{4}\right\rangle \\
& \Phi_{2}^{\dagger} \Phi_{2}\left|n_{1}, n_{2}, n_{3}, n_{4}\right\rangle=n_{2}\left|n_{1}, n_{2}, n_{3}, n_{4}\right\rangle \\
& \Phi_{3}^{\dagger} \Phi_{3}\left|n_{1}, n_{2}, n_{3}, n_{4}\right\rangle=-n_{3}\left|n_{1}, n_{2}, n_{3}, n_{4}\right\rangle \\
& \Phi_{4}^{\dagger} \Phi_{4}\left|n_{1}, n_{2}, n_{3}, n_{4}\right\rangle=-n_{4}\left|n_{1}, n_{2}, n_{3}, n_{4}\right\rangle .
\end{aligned}
$$

Comparing to the non-unitary representation of $H_{F}^{2,2}$ (Section 4.2.1), one may find that the spectra of $S O(1,4)(128)$ and $S O(3,2)$ Schwinger operators (101) are identical by the interchange, $n_{2} \leftrightarrow$ $n_{3}$. Thus, $H_{F}^{2,2}$ and $H_{F}^{4,0}$ are not "distinguished" only by their non-unitary representation ${ }^{17}$.

\footnotetext{
${ }^{17}$ The non-unitary representation (126) is naturally regarded as fully symmetric representation of $S U(2,2)$. Also, $k(117)$ is the $S U(2,2)$ invariant matrix. This suggests that the enhanced symmetry of $H_{F}^{4,0}$ is $S U(2,2)$. We will revisit this in Section 4.5.
} 


\subsubsection{Infinite dimensional unitary representation}

By the replacement $\Phi_{3} \leftrightarrow \Phi_{3}^{\dagger}$ and $\Phi_{4} \leftrightarrow \Phi_{4}^{\dagger}$, we can define new creation and annihilation operators that satisfy the usual commutation relations:

$$
\left[\Phi_{\alpha}, \Phi_{\beta}^{\dagger}\right]=\delta_{\alpha \beta}, \quad\left[\Phi_{\alpha}, \Phi_{\beta}\right]=\left[\Phi_{\alpha}^{\dagger}, \Phi_{\beta}^{\dagger}\right]=0 .
$$

With the newly defined Schwinger operator, the fuzzy coordinates on $H_{F}^{4,0}$ are represented as

$$
\begin{aligned}
& X^{1}=i \Phi_{1}^{\dagger} \Phi_{4}^{\dagger}+i \Phi_{2}^{\dagger} \Phi_{3}^{\dagger}-i \Phi_{1} \Phi_{4}-i \Phi_{2} \Phi_{3}, \\
& X^{2}=\Phi_{1}^{\dagger} \Phi_{4}^{\dagger}-\Phi_{2}^{\dagger} \Phi_{3}^{\dagger}+\Phi_{1} \Phi_{4}-\Phi_{2} \Phi_{3}, \\
& X^{3}=i \Phi_{1}^{\dagger} \Phi_{3}^{\dagger}-i \Phi_{2}^{\dagger} \Phi_{4}^{\dagger}-i \Phi_{1} \Phi_{3}+i \Phi_{2} \Phi_{4}, \\
& X^{4}=\Phi_{1}^{\dagger} \Phi_{3}^{\dagger}+\Phi_{2}^{\dagger} \Phi_{4}^{\dagger}+\Phi_{1} \Phi_{3}+\Phi_{2} \Phi_{4}, \\
& X^{5}=\Phi_{1}^{\dagger} \Phi_{1}+\Phi_{2}^{\dagger} \Phi_{2}+\Phi_{3} \Phi_{3}^{\dagger}+\Phi_{4} \Phi_{4}^{\dagger} .
\end{aligned}
$$

Again, the fuzzy coordinates $X^{1}, X^{2}, X^{3}$ and $X^{4}$ originally constructed by anti-hermitian gamma matrices become particle-number non-conserving operators. From (130), we have

$$
\begin{aligned}
& \eta_{a b} X^{a} X^{b}=-X^{1} X^{1}-X^{2} X^{2}-X^{3} X^{3}+X^{4} X^{4}+X^{5} X^{5}=\left(\Phi^{\dagger} k \Phi-2\right)\left(\Phi^{\dagger} k \Phi+2\right), \\
& X^{5}=\Phi_{1}^{\dagger} \Phi_{1}+\Phi_{2}^{\dagger} \Phi_{2}+\Phi_{3}^{\dagger} \Phi_{3}+\Phi_{4}^{\dagger} \Phi_{4}+2 .
\end{aligned}
$$

Their simultaneous eigenstates are

$$
\left.\mid n_{1}, n_{2}, n_{3}, n_{4}\right)=\frac{1}{\sqrt{n_{1} ! n_{2} ! n_{3} ! n_{4} !}}\left(\Phi_{1}^{\dagger}\right)^{n_{1}}\left(\Phi_{2}^{\dagger}\right)^{n_{2}}\left(\Phi_{3}^{\dagger}\right)^{n_{3}}\left(\Phi_{4}^{\dagger}\right)^{n_{4}}|\mathrm{vac}\rangle
$$

where $n_{1}, n_{2}, n_{3}, n_{4}$ are non-negative integers and $|\operatorname{vac}\rangle$ is defined as $\Phi_{1}|\operatorname{vac}\rangle=\Phi_{2}|\operatorname{vac}\rangle=\Phi_{3}|\operatorname{vac}\rangle=$ $\Phi_{4}|\mathrm{vac}\rangle=0$. The eigenvalues are given by

$$
\begin{aligned}
& \eta_{a b} X^{a} X^{b}=(n-2)(n+2)=n^{2}-4, \\
& X^{5}=n_{1}+n_{2}+n_{3}+n_{4}+2,
\end{aligned}
$$

with

$$
n \equiv n_{1}+n_{2}-n_{3}-n_{4} .
$$

For (semi-)positive definiteness of square of the radius, $n$ in (133a) should be taken as $n \geq 2$. With a given $n$, the spectra of $X^{5}$ read as

$$
X^{5}=n+2+2\left(n_{3}+n_{4}\right)=n+2, n+4, n+6, \cdots .
$$

Note that the range of the spectra of $X^{5}$ (135) is different from that of $H_{F}^{2,2}(112)$. This is consistent with the fact that $H_{F}^{4,0}$ corresponds to fuzzyfication (of the upper leaf) of two-leaf fourhyperboloid and $H_{F}^{2,2}$ corresponds to another four-fuzzy hyperboloid. In the language of $S O(1,4)$ representation theory, the present unitary representation of $H_{F}^{4,0}$ corresponds to the discrete series of $\operatorname{SO}(1,4)^{18}$.

\footnotetext{
${ }^{18}$ With use of $X^{a}(130)$, the $S O(1,4)$ generators are constructed as

$$
X^{a b} \equiv-i \frac{1}{4}\left[X^{a}, X^{b}\right]
$$
}


Similar to $H^{4,0}$, the isometry of $H^{1,3}\left(=d S^{4}\right)$ is given by $S O(1,4)$. Fuzzyfication of $H^{4,0}$ is realized by adopting the discrete series of $S O(1,4)$, while fuzzyfication of $H^{1,3}\left(=d S^{4}\right)$ is by the principal series $[42,43]^{19}$. (This is a higher dimensional analogue of the relations between $H_{F}^{2,0}$ and $H_{F}^{1,1}$ mentioned in the last paragraph of Section 3.2.2.)

\subsection{Enhanced Symmetry}

So far, everything is parallel between fuzzy two- and four-hyperboloids, except for the extra degeneracy of states in 4D case. Such "extra" degrees of freedom reflect symmetry enhancement particular to higher dimensional fuzzy hyperboloid.

\subsubsection{Enhanced algebra and internal structure}

Unlike fuzzy two-hyperboloid, the coordinates $X^{a}$ on fuzzy four-hyperboloid do not satisfy a closed algebra by themselves. (In the following, we treat $H_{F}^{2,2}$ and $H_{F}^{4,0}$ in a unified way : For $H_{F}^{2,2}, \eta^{a b}$ are taken as the $S O(3,2)$ metric, while for $H^{4,0}$, the $S O(1,4)$ metric.) The commutators of $X^{a}$ yield "new" operators $X^{a b}$;

$$
\left[X^{a}, X^{b}\right]=4 i X^{a b}
$$

Together with $X^{a b}, X^{a}$ satisfy the closed algebra

$$
\begin{aligned}
& {\left[X^{a b}, X^{c}\right]=i \frac{1}{2}\left(\eta^{a c} X^{b}-\eta^{b c} X^{a}\right)} \\
& {\left[X^{a b}, X^{c d}\right]=i\left(\eta^{a c} X^{b d}-\eta^{a d} X^{b c}+\eta^{b d} X^{a c}-\eta^{b c} X^{a d}\right)}
\end{aligned}
$$

where

$$
X^{a b}=-i \frac{1}{4}\left[X^{a}, X^{b}\right]=\Phi^{\dagger} k \gamma^{a b} \Phi
$$

with 20

$$
\gamma^{a b}=-i \frac{1}{4}\left[\gamma^{a}, \gamma^{b}\right]
$$

and the $S O(1,4)$ quadratic Casimir is given by

$$
\sum_{a<b} X_{a b} X^{a b}=\frac{1}{2}\left(\Phi^{\dagger} k \Phi+2\right)\left(\Phi^{\dagger} k \Phi-2\right)=\frac{1}{2}(n+2)(n-2) .
$$

In the Dixmier notation[70], with $S O(1,4)$ generators $L_{a b}$, the quadratic Casimir and its eigenvalues are generally given by

$$
C_{2}=\sum_{a<b} L_{a b} L^{a b}=p(p+1)+(q+1)(q-2) .
$$

The eigenvalues of the unitary representation of (137) are realized as the discrete series by the choice of $p=q=\frac{n}{2}$ in (138). See Refs. [43, 70, 71, 72, 73] for more details about the representation theory of $S O(1,4)$.

${ }^{19}$ Different from the present work, in Refs.[42, 43] $S O(1,4)$ quartic Casimir made of Pauli-Lubanski vectors was adopted to define fuzzy $d S^{4}$. Such fuzzy $d S^{4}$ construction provides another natural generalization of fuzzy hyperboloid.

${ }^{20} \gamma^{a b}(144)$ satisfy

$$
\begin{aligned}
& {\left[\gamma^{a b}, \gamma^{c}\right]=i\left(\eta^{a c} \gamma^{b}-\eta^{b c} \gamma^{a}\right),} \\
& {\left[\gamma^{a b}, \gamma^{c d}\right]=i\left(\eta^{a c} \gamma^{b d}-\eta^{a d} \gamma^{b c}+\eta^{b d} \gamma^{a c}-\eta^{b c} \gamma^{a d}\right) .}
\end{aligned}
$$


Define $X^{A B}(A, B=1,2, \cdots, 6)$ as

$$
\begin{array}{lll}
X^{A B} \equiv X^{a} & \text { for } & (A, B)=(6, a), \\
X^{A B} \equiv X^{a b} & \text { for } & (A, B)=(a, b) .
\end{array}
$$

The above algebras, (139) and (140), are concisely rewritten as

$$
\left[X^{A B}, X^{C D}\right]=i\left(\eta^{A C} X^{B D}-\eta^{A D} X^{B C}+\eta^{B D} X^{A C}-\eta^{B C} X^{A D}\right),
$$

where $\eta^{A B}$ denotes $S O(4,2)$ or $S O(2,4)$ metric corresponding to $H_{F}^{2,2}$ or $H_{F}^{4,0} 21$. In either cases, $X^{A B}$ satisfy the $S U(2,2)(\simeq S O(4,2) \simeq S O(2,4))$ algebra. Thus, the total algebras that correspond to $H_{F}^{2,2}$ and $H_{F}^{4,0}$ are identically given by $S U(2,2)$. This is a non-compact counterpart [32] of the enhanced $S U(4)$ algebra of fuzzy four-sphere [7, 11, 12]:

$$
\begin{array}{cccc}
\text { Fuzzy manifold } & S_{F}^{4} & H_{F}^{4,0} & H_{F}^{2,2} \\
\text { Original algebra } & S O(5) & S O(1,4) & S O(3,2) \\
\text { Enhanced algebra } & S O(6) \simeq S U(4) & S O(2,4) \simeq S U(2,2) & S O(4,2) \simeq S U(2,2)
\end{array}
$$

Thus, the total fuzzy coordinates of fuzzy four-hyperboloid may be considered as $X^{a}$ and $X^{a b}$. Existence of $X^{a b}$ suggests "internal structure" of fuzzy four-hyperboloid. With a fixed latitude of $X^{5}$, the remaining $X^{\mu}(\mu=1,2,3,4)$ construct $X^{\mu \nu}=-i \frac{1}{4}\left[X^{\mu}, X^{\nu}\right](\mu, \nu=1,2,3,4)$ that satisfy $S O(2,2)$ or $S O(4)$ algebra for $H_{F}^{2,2}$ or $H_{F}^{4,0}$. Notice that both of these are semi-simple algebras, i.e. $s o(2,2) \simeq s u(1,1) \oplus s u(1,1)$ and $s o(4) \simeq s u(2) \oplus s u(2)$. Since $S U(1,1)$ and $S U(2)$ are the algebras to define $H_{F}^{2,0}$ and $S_{F}^{2}$ respectively, such algebraic decomposition implies that at a fixed latitude on fuzzy four hyperboloid, there exists "fuzzy bundle" made of two fuzzy two-hyperboloids for $H_{F}^{2,2}$, and two fuzzy two-spheres for $H_{F}^{4,0}$. Indeed, the fuzzy four-hyperboloids have the following geometries

$$
\left.H_{F}^{2,2}(n)\right|_{X^{5}} \sim H_{F}^{2,0}\left(\frac{n+X^{5}}{2}\right) \oplus H_{F}^{2,0}\left(\frac{n-X^{5}}{2}\right)
$$

and

$$
\left.H_{F}^{4,0}(n)\right|_{X^{5}} \sim S_{F}^{2}\left(\frac{n+X^{5}}{2}\right) \oplus S_{F}^{2}\left(\frac{n-X^{5}}{2}\right)
$$

where $H_{F}^{2,0}(n)$ and $S_{F}^{2}(n)$ denote the fuzzy $H^{2,0}$ and fuzzy $S^{2}$ with radius $n$, respectively. According to the similar arguments in fuzzy four-sphere [19], it can be shown that the radii of fuzzy

These relations are readily derived by using $\left\{\gamma^{a}, \gamma^{b}\right\}=2 \eta^{a b}$ and the formula

$$
[A B, C D]=A\{B, C\} D-A C\{B, D\}+\{A, C\} D B-C\{A, D\} B .
$$

\footnotetext{
${ }^{21}$ If we defined $X^{A B}$ as $X^{A B} \equiv i X^{a}$ for $(A, B)=(6, a)$ and $X^{A B} \equiv X^{a b}$ for $(A, B)=(a, b), X^{A B}$ would become $S O(3,3)$ generators. However, in this case, hermiticity of $X^{A B}$ is not coherent: $X^{a b}$ are hermitian but $X^{6 a}$ are anti-hermitian. Then, we adopt the definition (145).
} 
hyperboloid- and fuzzy sphere-bundles are specified by $n$ and $X^{5}$ as given by (147) and (148). Eq.(147) indicates that the Hilbert space of the $S O(3,2)$ representation (100) which lives on the latitude $X^{5}$ of $H_{F}^{2,2}$ with radius $n$ is given by the direct-sum of the Hilbert spaces of two $S U(1,1)$ representations (70) of fuzzy two-hyperboloids with radii $\left(n+X^{5}\right) / 2$ and $\left(n-X^{5}\right) / 2$. Similarly, Eq.(148) represents that the Hilbert space of the $S O(1,4)$ representation (126) which lives on the latitude $X^{5}$ of $H_{F}^{4,0}$ with radius $n$ is given by the direct-sum of the Hilbert spaces of two $S U(2)$ representations of fuzzy two-hyperboloids with radii $\left(n+X^{5}\right) / 2$ and $\left(n-X^{5}\right) / 2{ }^{22}$. Around the "north pole" $X^{5} \sim n,(147)$ and (148) are respectively reduced to $\left.H_{F}^{2,2}(n)\right|_{X^{5} \sim n} \sim H_{F}^{2,0}(n)$ and $\left.H_{F}^{4,0}(n)\right|_{X^{5} \sim 0} \sim S_{F}^{2}(n)$, and hence $H_{F}^{2,2}$ and $H_{F}^{4,0}$ are locally expressed as

$$
H_{F}^{2,2}(n) \sim H^{2,2}(n) \otimes H_{F}^{2,0}(n)
$$

and

$$
H_{F}^{4,0}(n) \sim H^{4,0}(n) \otimes S_{F}^{2}(n) .
$$

Thus, $H_{F}^{2,2}$ is locally equivalent to fibration of fibre $H_{F}^{2,0}$ over the basemanifold $H^{2,2}$ and similarly, $H_{F}^{4,0}$ is equivalent to fibration of $S_{F}^{2}$ over $H^{4,0}$. With use of the original symmetries of the hyperbolic basemanifolds, (149) and (150) can be expressed as

$$
H_{F}^{2,2} \simeq S O(3,2) / U(1,1)
$$

and

$$
H_{F}^{4,0} \simeq S O(1,4) / U(2)
$$

where we utilized $S O(3,2) / U(1,1) \sim H^{2,2} \otimes S O(2,2) / U(1,1) \sim H^{2,2} \otimes S U(1,1) / U(1)$ for (151), and $S O(1,4) / U(2) \sim H^{4,0} \otimes S O(4) / U(2) \sim H^{4} \otimes S U(2) / U(1)$ for $(152)$.

The non-compact Hopf maps are a "classical" counterpart of the fuzzy hyperboloids, and then the corresponding structures can also be observed in the geometry of the non-compact Hopf maps. To see this, consider the total manifold of the split 2nd Hopf map, $H^{4,3}$, and its corresponding symplectic manifold $\mathbb{C} P^{1,2}[32]^{23}$

$$
\mathbb{C} P^{1,2} \simeq H^{4,3} / S^{1} \sim H^{2,2} \otimes H^{2,1} / S^{1} \simeq H^{2,2} \otimes H^{2,0} .
$$

One may find that the last expression on the right-hand side of (153) corresponds to (149). Similarly for the hybrid 2nd Hopf map, we have

$$
\mathbb{C} P^{1,2} \simeq H^{4,3} / S^{1} \sim H^{4,0} \otimes S^{3} / S^{1} \simeq H^{4,0} \otimes S^{2} .
$$

Again, the last expression on the right-hand side of (154) corresponds to (150). Thus, the indefinite complex projective space $\mathbb{C} P^{1,2}$ is considered as the classical counterpart of the $H_{F}^{2,2}$ and $H_{F}^{4,0}$ (see Appendix $\mathrm{C}$ for $\mathbb{C} P^{p, q}$ ). Meanwhile, $\mathbb{C} P^{1,2}$ is represented by the coset:

$$
\mathbb{C} P^{1,2} \simeq S U(2,2) / U(1,2) .
$$

\footnotetext{
${ }^{22}$ Non-unitary representations of $H_{F}^{2,2}(100)$ and $H_{F}^{4,0}(126)$ carry four quantum numbers, $n_{1}, n_{2}, n_{3}$ and $n_{4}$. Two of them specify the radius of fuzzy four-hyperboloid $n\left(=n_{1}+n_{2}+n_{3}+n_{4}\right)$ and the spectrum of $X^{5}$. The other two specify two latitudes of the two fuzzy hyperboloid- or two fuzzy sphere-bundles.

${ }^{23}$ Eq. (153) implies that $H^{4,3}$ can be expressed as two distinct fibrations, $H^{4,3} \sim \mathbb{C} P^{1,2} \otimes S^{1}$ and $H^{4,3} \sim H^{2,2} \otimes H^{2,1}$. We reconsider this in the context of lowest Landau level physics in Section 6.2.
} 


\begin{tabular}{|c||c|c|c|c|c|}
\hline Symmetry & Original manifolds & Hopf Maps & $k$ matrix & Enhanced Algebra & Fuzzy Manifolds \\
\hline \hline$S O(5)$ & $S^{4}=H^{0,4}$ & Compact & Hermite & $S O(6) \simeq S U(4)$ & $S_{F}^{4} \simeq \mathbb{C} P^{3,0}$ \\
\hline$S O(4,1)$ & $d S^{4}=H^{1,3}$ & $/$ & Anti-hermite & $/$ & $/$ \\
\hline$S O(3,2)$ & $H^{2,2}$ & Split & Hermite & $S O(2,4) \simeq S U(2,2)$ & $H_{F}^{2,2} \simeq \mathbb{C} P^{1,2}$ \\
\hline$S O(2,3)$ & $A d S^{4}=H^{3,1}$ & $/$ & Anti-hermite & $/$ & $/$ \\
\hline$S O(1,4)$ & $E A d S^{4}=H^{4,0}$ & Hybrid & Hermite & $S O(4,2) \simeq S U(2,2)$ & $H_{F}^{4,0} \simeq \mathbb{C} P^{1,2}$ \\
\hline
\end{tabular}

Table 2: Fuzzyfication of four-hyperboloids and related properties.

The $S U(2,2)$ symmetry naturally appears as the isometry of $\mathbb{C} P^{1,2}$. This is another way of understanding the appearance of the $S U(2,2)$ in the geometry of fuzzy $H_{F}^{2,2}$ and fuzzy $H^{4,0}$.

The relevant mathematical structures of $4 \mathrm{D}$ fuzzy sphere and fuzzy hyperboloids are summarized in Table 2 .

\subsubsection{Quantum fluctuations of geometry}

Physically, the symmetry enhancement is brought by quantum fluctuations of the geometry on fuzzy hyperboloid $[5,6]$. To see this, we first introduce the coherent state $|\omega\rangle$ to satisfy

$$
\eta_{a b} x^{a} X^{b}|\omega\rangle=n|\omega\rangle
$$

Here, $X^{a}$ and $x^{a}$ are coordinates on fuzzy and classical four-hyperboloid respectively, and $\eta_{a b}$ is the corresponding indefinite metric, i.e. the $S O(1,4)$ metric for $H_{F}^{4,0}$ or the $S O(3,2)$ metric for $H_{F}^{2,2}$. The coherent state is derived as

$$
|\omega\rangle=\frac{1}{\sqrt{n !}}\left(\Phi^{\dagger} k \phi\right)^{n}|0\rangle,
$$

where $k$ is the hermitianizing matrix, $\Phi$ is the Schwinger operator whose components satisfy $\left[\Phi_{\alpha}, \Phi_{\beta}^{\dagger}\right]=k_{\alpha \beta},\left[\Phi_{\alpha}, \Phi_{\beta}\right]=\left[\Phi_{\alpha}^{\dagger}, \Phi_{\beta}^{\dagger}\right]=0$, and $\phi$ is the non-compact Hopf spinor. The dual state of $|\omega\rangle$ is given by

$$
\langle\omega|=\frac{1}{\sqrt{n !}}\langle 0|\left(\phi^{\dagger} k \Phi\right)^{n},
$$

which satisfies $\langle\omega \mid \omega\rangle=1$. With the coherent state, one-point functions are derived as

$$
\left\langle\omega\left|X^{a}\right| \omega\right\rangle=n x^{a} .
$$

Thus, one-point functions reduce to coordinates on the corresponding classical hyperboloid. Meanwhile, two-point functions are calculated as

$$
\left\langle\omega\left|X^{a} X^{b}\right| \omega\right\rangle=n^{2} x^{a} x^{b}+n\left(-x^{a} x^{b}+2 i x^{a b}+\eta^{a b}\right),
$$

where $x^{a b}$ are defined by

$$
x^{a b} \equiv \frac{1}{n}\left\langle\omega\left|X^{a b}\right| \omega\right\rangle .
$$


$x^{a b}$ do not have any counterpart in coordinates on the classical hyperboloid. Since $x^{a}$ and $x^{a b}$ are the expectation values of the $S U(2,2)$ operators, they amount to coordinates on $\mathbb{C} P^{1,2}$. The subdominant second term of the order $n$ on the right-hand side of (160) signifies quantum fluctuations, and it includes $x^{a b}$ : the $\mathbb{C} P^{1,2}$ geometry. In the "classical limit" $n \rightarrow \infty$, we see that (160) indeed reduces to its classical counterpart $\left\langle\omega\left|X^{a} X^{b}\right| \omega\right\rangle \rightarrow n^{2} x^{a} x^{b}$, while in the quantum limit $n \simeq O(1)$, the particular $\mathbb{C} P^{1,2}$ coordinates $x^{a b}$ become comparable to the classical term $x^{a} x^{b}$ of the order $n^{2}$, indicating that quantum fluctuations on fuzzy hyperboloid smear the original hyperbolic geometry to generate a new enhanced geometry of $\mathbb{C} P^{1,2}$.

\section{Even Higher Dimensional Generalization of Fuzzy Hyperboloid}

For the previous realization of fuzzy hyperboloids, we utilized gamma matrices of low dimensional indefinite orthogonal groups. In this section, we extend the previous analysis to construct even higher dimensional fuzzy hyperboloids based on general indefinite gamma matrices (Section 5.1 and 5.2). We also investigate fuzzy-bundle structure of fuzzy hyperboloids (Section 5.3).

\subsection{Hierarchical structure of fuzzy ultra-hyperboloid}

Remember, in the case of fuzzy $H^{2,2}$, its coordinates are essentially given by the $S O(3,2)$ gamma matrices. Therefore, it may be natural to define general fuzzy $H^{2 p, 2 q}$ for their coordinates to satisfy the algebra of $S O(2 q+1,2 p)$ gamma matrices. Such fuzzy coordinates do not close algebra by themselves without introducing $S O(2 q+1,2 p)$ generators. In total, they amount to $S O(2 q+2,2 p)$ algebra, and in this sense the $S O(2 q+2,2 p)$ algebra can be considered as the total algebra of fuzzy $H^{2 p, 2 q}$. For fuzzy four-hyperboloids, $H_{F}^{2,2}$ and $H_{F}^{4,0}$, their total algebras were identically given by $S O(2,4) \simeq S O(4,2) \simeq S U(2,2)$. Thus, besides the original fuzzy coordinates that reduce to the classical coordinates of the original hyperboloid $H^{2 p, 2 q}$, the closure of the algebra "requires" an extra fuzzy space spanned by the $S O(2 q+1,2 p)$ algebra. Such newly introduced fuzzy space is considered as $H_{F}^{2 p, 2 q-2}$ as we shall see below. On a fixed latitude of the basemanifold of $H^{2 p, 2 q}$, which is simply realized by taking an eigenvalue of the fuzzy coordinate $X^{2 p+2 q+1}$, the commutators between the remaining fuzzy coordinates $X^{\mu}(\mu=1,2, \cdots, 2 p+2 q)$ yield the $S O(2 q, 2 p)$ generators. Thus at each latitude of the original hyperboloid, the $S O(2 q, 2 p)$ algebra is defined. This can be regarded as a sort of "fibration". The $S O(2 q, 2 p)$ algebra is the defining algebra of fuzzy $H^{2 p, 2 q-2}$ as discussed above, and then "SO(2q,2p)"-fiber geometrically indicates

$H_{F}^{2 p, 2 q-2}$. In this sense, $H_{F}^{2 p, 2 q}$ is understood as such a fuzzy manifold whose basemanifold is $H^{2 p, 2 q}$ and fibre is $H_{F}^{2 p, 2 q-2}$ :

$$
H_{F}^{2 p, 2 q} \sim H^{2 p, 2 q} \otimes H_{F}^{2 p, 2 q-2}
$$

for $q \neq 0$. Consequently, fuzzy hyperboloid is considered as a fibration of lower dimensional fuzzy hyperboloid-bundle on a hyperbolic basemanifold. We designate such hierarchical geometry (162) as the hyperbolic hierarchy. This is a generalization of the split Hopf map, since (162) for $p=q=1$ reduces to $(153)$.

For $q=0$, a special care is needed. In the case $q=0$, the "fibre" is given by $S O(2 p)$ algebra 
that defines $S_{F}^{2 p-2}$. Then, the relation (162) is modified to give

$$
H_{F}^{2 p, 0} \sim H^{2 p} \otimes S_{F}^{2 p-2} .
$$

We designate the structure (163) as the hybrid hierarchy, since (163) is a generalization of the hybrid Hopf map, in the sense that (163) for $p=2$ reduces to the hybrid Hopf map (154).

The ultra-hyperboloids thus "contain" lower dimensional fuzzy hyperboloids (or spheres) as their fuzzy-fibre. Inversely, higher dimensional hyperboloids can be constructed by low dimensional fuzzy manifolds. Such geometrical structure is called the dimensional hierarchy [27, 12].

\subsubsection{Hyperbolic hierarchy: Construction I}

We argue the dimensional hierarchy in view of the structure of indefinite gamma matrices. The gamma matrices of $S O(2 q+1,2 p)$ are "constructed" by those of $S O(2 q-1,2 p)$. We first introduce the $S O(2 q-1,2 p)$ gamma matrices $\gamma^{i}(i=1,2, \cdots, 2 p+2 q-1)$ that satisfy

$$
\left\{\gamma^{i}, \gamma^{j}\right\}=2 \eta^{i j}
$$

with $\eta_{i j}=\operatorname{diag}(\overbrace{+,+, \cdots,+}^{2 q-1}, \overbrace{-,-, \cdots,-}^{2 p})$. The hermitianizing matrix $k$ is hermitian, and $k \gamma^{i}$ are hermitianized gamma matrices. The $S O(2 q+1,2 p)$ gamma matrices are constructed as

$$
\Gamma^{i}=-\gamma^{i} \otimes \sigma^{2}=\left(\begin{array}{cc}
0 & i \gamma^{i} \\
-i \gamma^{i} & 0
\end{array}\right), \quad \Gamma^{2 p+2 q}=\left(\begin{array}{ll}
0 & \mathbf{1} \\
\mathbf{1} & 0
\end{array}\right), \quad \Gamma^{2 p+2 q+1}=\left(\begin{array}{cc}
\mathbf{1} & 0 \\
0 & -\mathbf{1}
\end{array}\right),
$$

which satisfy

$$
\left\{\Gamma^{a}, \Gamma^{b}\right\}=2 \eta^{a b}
$$

with $\eta^{a b}=\left(\eta^{i j},+,+\right)$. The construction of $S O(3,2)$ gamma matrices (86) is the simplest demonstration of the formula (165) . The hermitianizing matrix $K$ is also given by

$$
K=k \otimes 1_{2}=\left(\begin{array}{ll}
k & 0 \\
0 & k
\end{array}\right)
$$

and indeed the $S O(2 q+1,2 p)$ gamma matrices become hermitian:

$$
K \Gamma^{i}=\left(\begin{array}{cc}
0 & i k \gamma^{i} \\
-i k \gamma^{i} & 0
\end{array}\right), \quad K \Gamma^{2 p+2 q}=\left(\begin{array}{ll}
0 & k \\
k & 0
\end{array}\right), \quad K \Gamma^{2 p+2 q+1}=\left(\begin{array}{cc}
k & 0 \\
0 & -k
\end{array}\right) .
$$

In this way, the $S O(2 q+1,2 p)$ gamma matrices are constructed by the $S O(2 q-1,2 p)$ gamma matrices. Since the gamma matrices correspond to coordinates of fuzzy hyperboloid, in the language of geometry, such hierarchical structure suggests that $H_{F}^{2 p, 2 q}$ contains $H_{F}^{2 p, 2 q-2}$ as its internal fuzzy space. This agrees with the observation (162). 


\subsubsection{Hybrid hierarchy: Construction II}

The $S O(2 p+1,2 q)$ gamma matrices can be constructed from the $S O(2 q-1,2 p)$ gamma matrices $\gamma^{i}$ as

$$
\Gamma^{i}=\gamma^{i} \otimes i \sigma^{1}=\left(\begin{array}{cc}
0 & i \gamma^{i} \\
i \gamma^{i} & 0
\end{array}\right), \quad \Gamma^{2 p+2 q}=\left(\begin{array}{cc}
0 & \mathbf{1} \\
-\mathbf{1} & 0
\end{array}\right), \quad \Gamma^{2 p+2 q+1}=\left(\begin{array}{cc}
\mathbf{1} & 0 \\
0 & -\mathbf{1}
\end{array}\right),
$$

which satisfy

$$
\left\{\Gamma^{a}, \Gamma^{b}\right\}=2 \eta^{a b}
$$

with $\eta^{a b}=\left(-\eta^{i j},-,+\right)$. The construction of $S O(1,4)$ gamma matrices $(116)$ is the simplest demonstration of the formula (169). The hermitianizing matrix $K$ is given by

$$
K=k \otimes \sigma^{3}=\left(\begin{array}{cc}
k & 0 \\
0 & -k
\end{array}\right)
$$

and the $S O(2 p+1,2 q)$ gamma matrices are hermitianized as

$$
K \Gamma^{i}=\left(\begin{array}{cc}
0 & i k \gamma^{i} \\
-i k \gamma^{i} & 0
\end{array}\right), \quad K \Gamma^{2 p+2 q}=\left(\begin{array}{ll}
0 & k \\
k & 0
\end{array}\right), \quad K \Gamma^{2 p+2 q+1}=\left(\begin{array}{cc}
k & 0 \\
0 & k
\end{array}\right) .
$$

In particular $p=0$, the $S O(1,2 q)$ gamma matrices are constructed by the $S O(2 q-1)$ gamma matrices: With $S O(2 q-1)$ gamma matrices $\gamma^{i}$ satisfying $\left\{\gamma^{i}, \gamma^{j}\right\}=2 \delta^{i j}$, we have

$$
K=\mathbf{1} \otimes \sigma^{3}=\left(\begin{array}{cc}
1 & 0 \\
0 & -1
\end{array}\right)
$$

and

$$
K \Gamma^{i}=\left(\begin{array}{cc}
0 & i \gamma^{i} \\
-i \gamma^{i} & 0
\end{array}\right), \quad K \Gamma^{2 p+2 q}=\left(\begin{array}{ll}
0 & \mathbf{1} \\
\mathbf{1} & 0
\end{array}\right), \quad K \Gamma^{2 p+2 q+1}=\left(\begin{array}{ll}
\mathbf{1} & 0 \\
0 & \mathbf{1}
\end{array}\right) .
$$

Such $S O(2 q-1)$ gamma matrix structure is consistent with the above observation that $H_{F}^{2 p, 0}$ contains $S_{F}^{2 p-2}$ as its internal fuzzy space (163).

\subsubsection{Examples in low dimension}

One can construct gamma matrices of $S O$ (odd,even) in any higher dimensions by following the method in Section 5.1.1 (Construction I) and the one in Section 5.1.2 (Construction II) from either $S O(3)$ or $S O(1,2)$ gamma matrices ${ }^{24}$. In low dimensions, we have

\footnotetext{
${ }^{24}$ The hermitianizing matrix of $S O(2 q+1,2 p)$ is also constructed by repeatedly applying the procedure (167) or (173) to the hermitianizing matrix of $S O(1,2)$ or $S O(3)$, i.e. $\sigma^{3}$ or $1_{2}$. Then, one finds that the hermitianizing matrix of $S O(2 q+1,2 p)$ is unitarily equivalent to the split-signature diagonal matrix, $K=\operatorname{diag}(\overbrace{+, \cdots,+,}^{2^{p+q-1}} \overbrace{-, \cdots,-}^{2^{p+q-1}})$.
} 


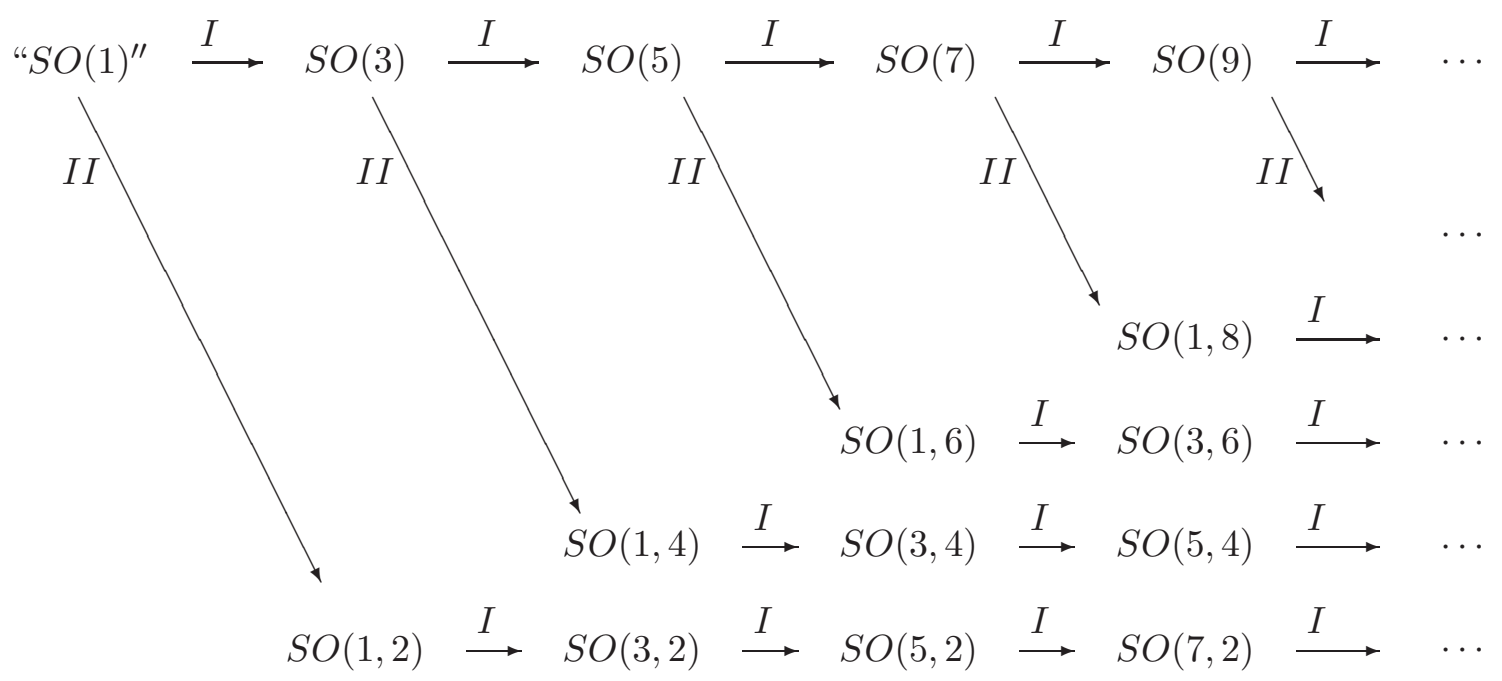

where $I$ and $I I$ denote "Construction I and II", and the gamma matrix of "SO(1)" is defined as -1 to construct $S O(3)$ gamma matrix by (165). For the construction of fuzzy ultra-hyperboloid $H_{F}^{2 p, 2 q}, S O(2 q+1,2 p)$ gamma matrices are utilized, and hence the above hierarchical structure of indefinite gamma matrices suggests the dimensional hierarchy of fuzzy ultra-hyperboloids:

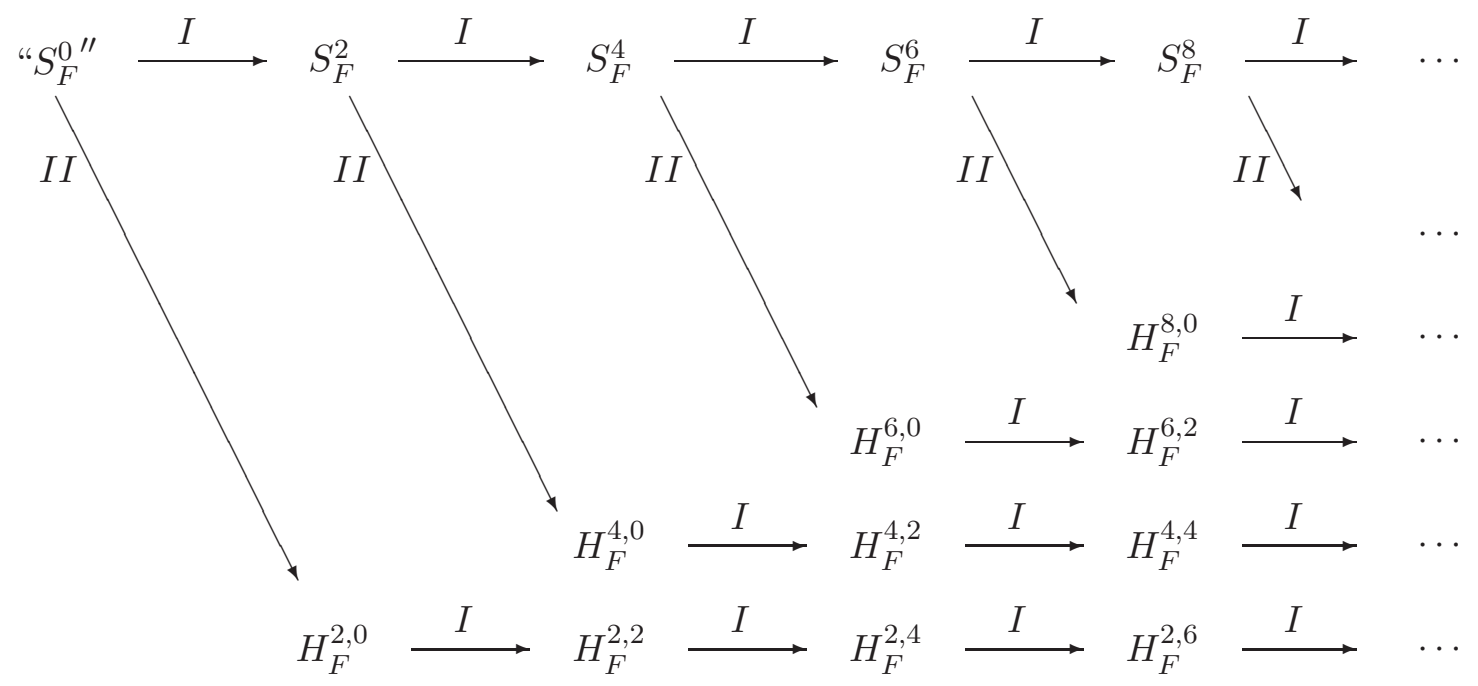

We have seen that the non-unitary representation of $H_{F}^{2 p, 2 q}(p+q \leq 2)$ is given by the finite dimensional representation that is equal to the fully symmetric representation of the fuzzy sphere $S_{F}^{2 p+2 q+1}$. Therefore, non-unitary finite dimensional representation for arbitrary fuzzy ultra-hyperboloids can be readily constructed by applying a similar technique developed in the analysis of fuzzy spheres [13, 74]. For unitary infinite dimensional representation in low dimensions, we have seen that the unitary representation made by the Schwinger operators corresponds to 
discrete series of the corresponding indefinite orthogonal groups. According to Harish-Chandra's equal-rank condition [75], arbitrary $S O(2 q+1,2 p)$ group generally accommodates discrete series ${ }^{25}$. Then, we can adopt the $S O(2 q+1,2 p)$ discrete series for unitary construction of $H_{F}^{2 p, 2 q}$.

\subsection{Classical counterpart}

Here, we introduce a coset for the "classical limit" of fuzzy hyperboloid. The classical counterpart of fuzzy two-hyperboloid, $H_{F}^{2,0}$, is given by $H_{F}^{2,0} \simeq S O(1,2) / U(1)$, while those of fuzzy fourhyperboloids are $H_{F}^{2,2} \simeq S O(3,2) / U(1,1)$ and $H_{F}^{4,0} \simeq S O(1,4) / U(2)$. Furthermore, from [7], we know that the classical counterpart of fuzzy sphere is $H_{F}^{0,2 p}=S_{F}^{2 p} \simeq S O(2 p+1) / U(p)$. Therefore, one may infer the coset of fuzzy hyperboloid as

$$
H_{F}^{2 p, 2 q} \simeq S O(2 q+1,2 p) / U(q, p) \simeq S O(2 p, 2 q+1) / U(p, q) .
$$

For $q \neq 0,(175)$ yields

$$
H_{F}^{2 p, 2 q} \sim H^{2 p, 2 q} \otimes S O(2 p, 2 q) / U(p, q),
$$

where $H^{2 p, 2 q} \simeq S O(2 p, 2 q+1) / S O(2 p, 2 q)$ was used. Thus, $H_{F}^{2 p, 2 q}$ is regarded as a manifold whose basemanifold is $H^{2 p, 2 q}$ and its fibre is represented by the coset $S O(2 p, 2 q) / U(p, q)$. From the relation

$$
S O(2 p, 2 q) / S O(2 p, 2 q-1) \simeq U(p, q) / U(p, q-1)\left(\simeq H^{2 p, 2 q-1}\right),
$$

one may find that (176) implies

$$
H_{F}^{2 p, 2 q} \sim H^{2 p, 2 q} \otimes S O(2 p, 2 q-1) / U(p, q-1) \simeq H^{2 p, 2 q} \otimes H_{F}^{2 p, 2 q-2} .
$$

As a special case of (175), for $q=0$, we have

$$
H_{F}^{2 p, 0} \sim H^{2 p, 0} \otimes S O(2 p) / U(p) .
$$

Thus, $H_{F}^{2 p, 0}$ is locally given by the fibre-bundle of the fibre $S O(2 p) / U(p)$ over the basemanifold $H^{2 p, 0}$. From

$$
S O(2 p) / S O(2 p-1) \simeq U(p) / U(p-1)\left(\simeq S^{2 p-1}\right),
$$

we find that (179) further suggests

$$
H_{F}^{2 p, 0} \sim H^{2 p, 0} \otimes S O(2 p-1) / U(p-1) \simeq H^{2 p, 0} \otimes S_{F}^{2 p-2} .
$$

Thus, we confirmed that (175) reproduces the geometrical structure of fuzzy hyperboloid discussed in Section 5.1, and these results support the validity of (175). Due to the existence of internal fuzzy manifold, the dimension of $H_{F}^{2 p, 2 q}$ is not $2(p+q)$ but larger than that of $H^{2 p, 2 q}$ :

$$
\operatorname{dim}[S O(2 q, 2 p+1) / U(q, p)]=(p+q+1)(p+q) .
$$

The dimensions of $H_{F}^{2 p, 2 q}$ and $H^{2 p, 2 q}$ coincide only in the cases for $(p, q)=(1,0)$ and $(0,1)$, i.e. $H_{F}^{2,0}$ and $S_{F}^{2}$.

\footnotetext{
${ }^{25}$ For instance, see Refs.[76, 77, 78] for more about non-compact groups and also Refs.[79, 80] for unitary representation of the $S U(p, q)$ group made by Schwinger operator.
} 
In the following, we give several concrete examples of the dimensional hierarchy of fuzzy hyperboloids and their corresponding gamma matrices. We use the definition, $S_{F}^{2 p} \equiv H_{F}^{0,2 p}$ and $H_{F}^{2 p} \equiv H_{F}^{2 p, 0}$. For $p+q=1$, we have

$$
\begin{aligned}
& S O(3) \text { gamma mat. : } \quad S_{F}^{2} \sim H^{0,2}=S^{2}, \\
& S O(1,2) \text { gamma mat. : } H_{F}^{2} \sim H^{2,0}=H^{2} .
\end{aligned}
$$

For $p+q=2$,

$$
\begin{array}{ll}
S O(5) \text { gamma mat. }: & S_{F}^{4} \sim H^{0,4} \otimes H_{F}^{0,2} \sim S^{4} \otimes S^{2}, \\
S O(3,2) \text { gamma mat. }: & H_{F}^{2,2} \sim H^{2,2} \otimes H_{F}^{2,0} \sim H^{2,2} \otimes H^{2}, \\
S O(1,4) \text { gamma mat. }: & H_{F}^{4} \sim H^{4,0} \otimes S_{F}^{2} \sim H^{4} \otimes S^{2} .
\end{array}
$$

Note that these three respectively correspond to three kinds of the 2nd Hopf map. For $p+q=3$,

$$
\begin{array}{ll}
S O(7) \text { gamma mat. }: & S_{F}^{6} \sim H^{0,6} \otimes H_{F}^{0,4} \sim S^{6} \otimes S^{4} \otimes S^{2}, \\
S O(5,2) \text { gamma mat. }: & H_{F}^{2,4} \sim H^{2,4} \otimes H_{F}^{2,2} \sim H^{2,4} \otimes H^{2,2} \otimes H^{2}, \\
S O(3,4) \text { gamma mat. }: & H_{F}^{4,2} \sim H^{4,2} \otimes H_{F}^{4,0} \sim H^{4,2} \otimes H^{4} \otimes S^{2}, \\
S O(1,6) \text { gamma mat. }: & H_{F}^{6} \sim H^{6,0} \otimes S_{F}^{4} \sim H^{6} \otimes S^{4} \otimes S^{2} .
\end{array}
$$

For $p+q=4$,

$$
\begin{array}{ll}
S O(9) \text { gamma mat. }: & S_{F}^{8} \sim H^{0,8} \otimes H_{F}^{0,6} \sim S^{8} \otimes S^{6} \otimes S^{4} \otimes S^{2}, \\
S O(7,2) \text { gamma mat. }: & H_{F}^{2,6} \sim H^{2,6} \otimes H_{F}^{2,4} \sim H^{2,6} \otimes H^{2,4} \otimes H^{2,2} \otimes H^{2}, \\
S O(5,4) \text { gamma mat. }: & H_{F}^{4,4} \sim H^{4,4} \otimes H_{F}^{4,2} \sim H^{4,4} \otimes H^{4,2} \otimes H^{4} \otimes S^{2}, \\
S O(3,6) \text { gamma mat. }: & H_{F}^{6,2} \sim H^{6,2} \otimes H_{F}^{6} \sim H^{6,2} \otimes H^{6} \otimes S^{4} \otimes S^{2}, \\
S O(1,8) \text { gamma mat. }: & H_{F}^{8} \sim H^{8} \otimes S_{F}^{6} \sim H^{8} \otimes S^{6} \otimes S^{4} \otimes S^{2} .
\end{array}
$$

\subsection{Non-compact monopoles}

It is known that monopole gauge fields are realized as canonical connection of fibre-bundles of fuzzy spheres (see [19] as a review). Similarly, non-compact monopoles appear as associated connection of bundles of fuzzy hyperboloids.

\section{$5.4 \quad U(1)$ monopole on $H^{2,0}$}

As a warm-up, we introduce $U(1)$ monopole on $H^{2,0}$ from the 1st non-compact Hopf map. From the non-compact 1st Hopf map (51), the total manifold $H^{2,1}$ can be expressed as

$$
H^{2,1} \simeq H^{2,0} \otimes S^{1}
$$

Since $S^{1} \simeq U(1)$, the non-compact 1st Hopf map is physically related to $H^{2,0}$ in the $U(1)$ monopole background. Indeed, by inverting (58), the total manifold $H^{2,1}$ denoted by $\phi$ is represented as

$$
\phi=\frac{1}{\sqrt{2\left(1+x^{3}\right)}}\left(\begin{array}{c}
1+x^{3} \\
x^{1}+i x^{2}
\end{array}\right) e^{i \chi},
$$


where $x^{i}$ are coordinates on $H^{2,0}$ and $e^{i \chi}$ stands for $U(1)$ phase factor. The associated fibreconnection is derived as

$$
A=-i \phi^{\dagger} \sigma^{3} d \phi=d x^{i} A_{i}
$$

where

$$
A_{i}=\epsilon_{i j 3} \frac{x^{j}}{2\left(1+x^{3}\right)}
$$

The corresponding curvature is

$$
F_{i j}=\partial_{i} A_{j}-\partial_{j} A_{i}=-\frac{1}{2} \epsilon_{i j k} x^{k} .
$$

\subsection{1 $S O(2 p)$ monopole on $H^{2 p, 0}$}

Similarly, from the hybrid 2nd Hopf map, $H^{4,3}$ is expressed as

$$
H^{4,3} \simeq H^{4,0} \otimes S^{3} .
$$

Since $S^{3}$ is the group manifold corresponding to $S U(2)$, the hybrid 2nd Hopf map is closely related to the $S U(2)$ monopole gauge field on $H^{4,0}$. The total manifold $H^{4,3}$ denoted by $\psi$ in (120) is parameterized as

$$
\psi=M \phi,
$$

where $\phi$ is the $S O(3)$ Hopf spinor subject to the constraint $\phi^{\dagger} \phi=1$, representing $S^{3}$, and $M$ is $4 \times 2$ matrix given by

$$
M=\frac{1}{\sqrt{2\left(1+x^{5}\right)}}\left(\begin{array}{c}
\left(1+x^{5}\right) 1_{2} \\
\left(x^{4} 1_{2}-i x^{i} \sigma^{i}\right)
\end{array}\right) .
$$

Here, $x^{a}$ denote the coordinates on $H^{4,0}$ that satisfy $\eta_{a b} x^{a} x^{b}=-\sum_{\mu, \nu=1}^{4} \delta_{\mu \nu} x^{\mu} x^{\nu}+x^{5} x^{5}=1$. The connection of $S^{3}$-bundle is evaluated to give $S U(2)$ monopole gauge field on $H^{4,0}$ :

$$
A=-i M^{\dagger} k d M=\frac{1}{2\left(1+x^{5}\right)} \eta_{\mu \nu i} x^{\mu} d x^{\nu} \sigma^{i}
$$

where $\eta_{\mu \nu i}$ is 't Hooft tensor $\eta_{\mu \nu i} \equiv \epsilon_{\mu \nu i 4}-\delta_{\mu i} \delta_{\nu 4}+\delta_{\mu 4} \delta_{\nu i}\left(\epsilon_{1234}=1, \mu=1,2,3,4\right)$ [81], and $k$ is given by (117). The fuzzy ultra-hyperboloid $H_{F}^{2 p, 0}$ is a generalization of the hybrid Hopf maps and is locally given by $H_{F}^{2 p, 0} \sim H^{2 p, 0} \otimes S_{F}^{2 p-2}$. Following the above procedure, we can derive the connection of fuzzy fibre $S_{F}^{2 p-2}$ over $H^{2 p, 0}$. We introduce the " $S O(1,2 p)$ Hopf spinor" $\Psi$ as a classical counterpart of the fuzzy hyperboloid $H_{F}^{2 p, 0}$,

$$
\Psi=M \Phi,
$$

where $M$ denotes $2^{p+1} \times 2^{p}$ matrix given by

$$
M=\frac{1}{\sqrt{2\left(1+x^{2 p+1}\right)}}\left(\begin{array}{c}
\left(1+x^{2 p+1}\right) \mathbf{1} \\
x^{2 p} \mathbf{1}-i x^{i} \gamma^{i}
\end{array}\right) .
$$

Thus, the $S O(1,2 p)$ Hopf spinor $\Psi$ is generally constructed by (196) from the $S O(2 p-1)$ Hopf spinor $\Phi$. The simplest example of this construction is (193): from the $S O(3)$ Hopf spinor to the 
$S O(1,4)$ Hopf spinor. Here, $x^{a}$ signify coordinates on $H^{2 p, 0}$ and satisfy $\eta_{a b} x^{a} x^{b}=1$ with $S O(1,2 p)$ metric $\eta_{a b}=\operatorname{diag}(-,-, \cdots,-,+)(a, b=1,2, \cdots, 2 p+1), 1$ denotes $2^{p} \times 2^{p}$ unit matrix and $\gamma^{i}$ $(i=1,2, \cdots, 2 p-1)$ are $S O(2 p-1)$ gamma matrices that satisfy

$$
\left\{\gamma^{i}, \gamma^{j}\right\}=2 \delta^{i j}
$$

$\Phi$ in (196) stands for the coordinates on $S_{F}^{2 p-2}$, which satisfies $\Phi^{\dagger} \Phi=1$ [see Ref.[27] for realization of $\Phi]$. From the following properties

$$
M^{\dagger} K M=\mathbf{1}, \quad M^{\dagger} K \Gamma^{a} M=x^{a} \mathbf{1},
$$

with $K(173)$ and $\Gamma^{a}(174)$, one may readily see that $\Psi$ satisfies $\Psi^{\dagger} K \Psi=\Phi^{\dagger} \Phi=1$ and the "generalized" hybrid Hopf map

$$
\Psi^{\dagger} K^{a} \Psi=x^{a} .
$$

The connection of fuzzy bundle $S_{F}^{2 p-2}$ is calculated as

$$
A=-i M^{\dagger} K d M=d x^{a} k A_{a},
$$

where

$$
A_{\mu}=-\frac{1}{1+x^{2 p+1}} \sigma_{\mu \nu} x^{\nu}, \quad A_{2 p+1}=0 .
$$

Here, $\sigma_{\mu \nu}$ are the $S O(2 p)$ generators $^{26}$,

$$
\sigma_{i j}=-i \frac{1}{4}\left[\gamma_{i}, \gamma_{j}\right], \quad \sigma_{i, 2 n}=-\sigma_{2 p, i}=\frac{1}{2} \gamma_{i},
$$

with $\gamma_{i} \equiv-\gamma^{i}$. The field strength $F_{a b}=\partial_{a} A_{b}-\partial_{b} A_{a}-i\left[A_{a}, A_{b}\right]$ is derived as

$$
F_{\mu \nu}=-x_{\mu} A_{\nu}+x_{\nu} A_{\mu}+\sigma_{\mu \nu}, \quad F_{\mu, 2 p+1}=-F_{2 p+1, \mu}=\left(1+x^{2 p+1}\right) A_{\mu} .
$$

Thus, the connection of $S_{F}^{2 p-2}$-fibre represents the $S O(2 p)$ gauge field on $H^{2 p, 0}$.

\subsection{2 $S O(2 p, 2 q)$ monopole on $H^{2 p, 2 q}$}

In the case of the split 2nd Hopf map, the total manifold $H^{4,3}$ is locally expressed as

$$
H^{4,3} \sim H^{2,2} \otimes H^{2,1} .
$$

Since $H^{2,1} \simeq S U(1,1)$, the split 2 nd Hopf map is closely related to $H^{2,2}$ in the $S U(1,1)$ monopole background. The normalized $S O(3,2)$ spinor $\psi$ representing the total manifold $H^{4,3}(91)$ can be expressed as

$$
\psi=M \phi,
$$

\footnotetext{
${ }^{26} \sigma_{\mu \nu}$ satisfy the $S O(2 p)$ algebra

$$
\left[\sigma_{\mu \nu}, \sigma_{\rho \sigma}\right]=i\left(\delta_{\mu \rho} \sigma_{\nu \sigma}-\delta_{\mu \sigma} \sigma_{\nu \rho}-\delta_{\nu \rho} \sigma_{\mu \sigma}+\delta_{\nu \sigma} \sigma_{\mu \rho}\right) .
$$
}


where $M$ denotes $4 \times 2$ matrix of the form

$$
M=\frac{1}{\sqrt{2\left(1+x^{5}\right)}}\left(\begin{array}{c}
\left(1+x^{5}\right) 1_{2} \\
x^{4} 1_{2}-i x^{i} \tau_{i}
\end{array}\right) .
$$

Here, $x^{a}$ are coordinates on $H^{2,2}: \eta_{a b} x^{a} x^{b}=\sum_{\mu, \nu=1}^{4} \eta_{\mu \nu} x^{\mu} x^{\nu}+x^{5} x^{5}=1\left(\eta_{\mu \nu}=\operatorname{diag}(-,-,+,+)\right)$, and $\phi$ is the $S O(1,2)$ Hopf spinor that satisfies the condition of $H^{2,1}$-fibre, $\phi^{\dagger} \sigma^{3} \phi=1$. The associated connection of $H^{2,1}$-fibre is derived as

$$
A=-i M^{\dagger} k d M=-\frac{1}{2\left(1+x^{5}\right)} \eta_{\mu \nu i}^{\prime} \sigma^{3} \tau^{i} x^{\mu} d x^{\nu},
$$

where $\eta_{\mu \nu i}^{\prime}(\mu, \nu=1,2,3,4)$ is "split"-'t Hooft symbol:

$$
\eta_{\mu \nu i}^{\prime}=\epsilon_{\mu \nu i 4}-\eta_{\mu i} \eta_{\nu 4}+\eta_{\nu i} \eta_{\mu 4}
$$

with $\epsilon_{i j k 4} \equiv \epsilon_{i j k} . \tau^{i}$ in $(209)$ are the $S U(1,1)$ Pauli matrices $(54)$, and $(209)$ denotes $S U(1,1)$ monopole gauge field. The commutators of the $S U(1,1)$ gauge field (209) yield

$$
\left[A_{\mu}, A_{\nu}\right]=i \frac{1}{1+x^{5}}\left(x_{\mu} A_{\nu}-x_{\nu} A_{\mu}\right)+i \frac{1}{2} \frac{1-x^{5}}{1+x^{5}} \eta_{\mu \nu i}^{\prime} \tau^{i}
$$

and the field strength, $F_{a b}=\partial_{a} A_{b}-\partial_{b} A_{a}-i\left[A_{a}, A_{b}\right]$, satisfies

$$
F_{a c} F_{b}^{c}=-\left(\eta_{a c}-x_{a} x_{b}\right)\left(\frac{1}{2} \tau_{i}\right)^{2}-i F_{a b} .
$$

Eqs.(211) and (212) will be useful in Section 6.2.1.

The above analysis can readily be applied to higher dimensional fuzzy hyperboloid $H^{2 p, 2 q}$ $(q \neq 0)$. Corresponding to $H_{F}^{2 p, 2 q}(q \neq 0)$ which is locally given by $H_{F}^{2 p, 2 q} \sim H^{2 p, 2 q} \otimes H_{F}^{2 p, 2 q-2}$, we introduce the "SO( $2 q+1,2 p)$ Hopf spinor"

$$
\Psi=M \psi
$$

where $M$ denotes $2^{p+q+1} \times 2^{p+q}$ matrix of the form

$$
M=\frac{1}{\sqrt{2\left(1+x^{2 p+2 q+1}\right)}}\left(\begin{array}{c}
\left(1+x^{2 p+2 q+1}\right) \mathbf{1} \\
x^{2 p+2 q} \mathbf{1}-i x^{i} \gamma_{i}
\end{array}\right) .
$$

Here, $\gamma^{i}(i=1,2, \cdots, 2 p+2 q-1)$ are the $S O(2 q-1,2 p)$ gamma matrices, $x^{a}(a=1,2, \cdots, 2 p+$ $2 q+1)$ are coordinates on the basemanifold $H^{2 p, 2 q}$ that satisfy $\eta_{a b} x^{a} x^{b}=1$ with $S O(2 q+1,2 p)$ metric $\eta_{a b}$, and 1 denotes $2^{p+q} \times 2^{p+q}$ unit matrix. $\psi$ signifies the fuzzy-fibre $H_{F}^{2 p, 2 q-2}$. From the $S O(1,2 p)$ Hopf spinor $\psi$, we repeat the procedure (213) " $q$ times" to construct the $S O(2 q+1,2 p)$ Hopf spinor. (The $S O(1,2 p)$ Hopf spinor itself is constructed by the procedure discussed in Section 5.4.1.) From $\Psi$, a projection matrix $P$ is constructed as

$$
P=\Psi \Psi^{\dagger} K
$$


which satisfies $P^{2}=P$ and $P \Psi=\Psi . M(214)$ satisfies

$$
M^{\dagger} K M=k, \quad M^{\dagger} K \Gamma^{a} M=x^{a} k,
$$

where $K$ and $k$ are the hermitianizing matrices (167) and $\Gamma^{a}$ are $S O(2 q+1,2 p)$ gamma matrices (168). Here, $\Gamma^{a}$ and $\gamma^{i}$ respectively signify $S O(2 q+1,2 p)$ and $S O(2 q-1,2 p)$ gamma matrices related by (165). We obtain $\Psi^{\dagger} K \Psi=\psi^{\dagger} k \psi=1$ and the "generalized" split-Hopf map

$$
\Psi^{\dagger} K^{a} \Psi=x^{a} .
$$

The connection of fuzzy bundle $H_{F}^{2 p, 2 q-2}$ over $H^{2 p, 2 q}$ is derived as

$$
A=-i M^{\dagger} K d M=d x^{a} k A_{a}
$$

where $A_{a}(a=1,2, \cdots, 2 p+2 q+1)$ are

$$
A_{\mu}=-\frac{1}{1+x^{2 p+2 q+1}} \sigma_{\mu \nu} x^{\nu}, \quad A_{2 p+2 q+1}=0 .
$$

Here $\mu, \nu=1,2, \cdots, 2 p+2 q$, and $\sigma_{\mu \nu}$ are the $S O(2 p, 2 q)$ generators $^{27}$ given by

$$
\sigma_{i j}=-i \frac{1}{4}\left[\gamma_{i}, \gamma_{j}\right], \quad \sigma_{i, 2 p+2 q}=-\sigma_{2 p+2 q, i}=\frac{1}{2} \gamma_{i}
$$

with $i, j=1,2, \cdots, 2 p+2 q-1$. The field strength $F_{a b}=\partial_{a} A_{b}-\partial_{b} A_{a}-i\left[A_{a}, A_{b}\right]$ is calculated as

$$
F_{\mu \nu}=-x_{\mu} A_{\nu}+x_{\nu} A_{\mu}+\sigma_{\mu \nu}, \quad F_{\mu, 2 p+2 q+1}=-F_{2 p+2 q+1, \mu}=\left(1+x^{2 p+2 q+1}\right) A_{\mu} .
$$

The $S O(2 p, 2 q)$ monopole gauge field is thus induced as the connection of fuzzy bundle $H_{F}^{2 p, 2 q-2}$ over the basemanifold $H^{2 p, 2 q}$.

To summarize, the connection of fuzzy bundle of $H_{F}^{2 p, 2 q}$ physically corresponds to $S O(2 p, 2 q)$ monopole gauge field over the basemanifold $H^{2 q, 2 p}$. In particular for $(p, q)=(2,0)$, the gauge group of monopole is $S O(4,0) \simeq S U(2) \otimes S U(2)$ or $S U(2)$. Meanwhile, for $(p, q)=(1,1)$, the gauge group of monopole is $S O(2,2) \simeq S U(1,1) \otimes S U(1,1)$ or $S U(1,1)$.

\section{Fuzzy Hyperboloid realized as Lowest Landau Level}

The lowest Landau level physics illustrates close relations between monopoles and fuzzy geometry [27]. Here, we explore such relations for fuzzy two- (Section 6.1) and four-hyperboloids (Section $6.2)$.

\footnotetext{
${ }^{27} \sigma_{\mu \nu}$ satisfy

$$
\left[\sigma_{\mu \nu}, \sigma_{\rho \sigma}\right]=i\left(\eta_{\mu \rho} \sigma_{\nu \sigma}-\eta_{\mu \sigma} \sigma_{\nu \rho}-\eta_{\nu \rho} \sigma_{\mu \sigma}+\eta_{\nu \sigma} \sigma_{\mu \rho}\right),
$$
}

with the $S O(2 q, 2 p)$ metric, $\eta_{\mu \nu}$. 


\subsection{Lowest Landau level on two-hyperboloid}

First, we introduce one-particle mechanics on two-hyperboloid in $U(1)$ monopole background [41]. The Lagrangian is given by

$$
L=\frac{M}{2} \eta_{i j} \dot{x}^{i} \dot{x}^{j}+\dot{x}^{i} A_{i},
$$

with $\eta_{i j}=\operatorname{diag}(-,-,+)^{28}$. Here, $A_{i}$ denotes the $U(1)$ gauge field

$$
A_{i}=\frac{I}{2} \epsilon_{i j 3} \frac{x^{j}}{1+x^{3}}
$$

with monopole charge $I / 2$ ( $I$ is an integer). In the lowest Landau level $M \rightarrow 0$, the kinetic term is quenched and the Lagrangian reduces to

$$
L_{L L L}=\dot{x}^{i} A_{i}=-i I \phi^{\dagger} \sigma^{3} \frac{d}{d t} \phi
$$

where $\phi$ stands for the $S O(1,2)$ Hopf spinor with the normalization condition,

$$
\phi^{\dagger} \sigma^{3} \phi=1
$$

The canonical conjugate of $\phi$ is derived as

$$
\pi=\frac{\partial L_{L L L}}{\partial \dot{\phi}}=-i I \phi^{\dagger} \sigma^{3}
$$

Then, $\pi$ is not the time derivative of $\phi^{\dagger}$, but $\phi^{\dagger}$ itself. Therefore, when we impose the canonical quantization condition between $\phi$ and $\pi$,

$$
\left[\phi_{\alpha}, \pi_{\beta}\right]=i \delta_{\alpha \beta}
$$

$\phi^{*}$ is expressed as

$$
\phi^{*}=\frac{1}{I} \sigma^{3} \frac{\partial}{\partial \phi} .
$$

The normalization (226) becomes a constraint imposed on Hilbert space

$$
\phi^{t} \frac{\partial}{\partial \phi} f_{L L L}=I f_{L L L}
$$

which determines the lowest Landau level bases, $f_{L L L} \cdot f_{L L L}$ is given by the homogeneous polynomials of the Hopf spinor constructed by replacing the Schwinger operator in (70) with the Hopf spinor. Furthermore, in the lowest Landau level, $x^{i}(58)$ are effectively represented as

$$
X_{i}=\frac{1}{I} \phi^{t}\left(\tau_{i}\right)^{t} \frac{\partial}{\partial \phi}
$$

which satisfy

$$
\left[X^{i}, X^{j}\right]=\frac{1}{I} i \epsilon^{i j k} X_{k}
$$

\footnotetext{
${ }^{28}$ The corresponding Landau problem is investigated in Ref.[82]. See also [41, 83] for related works about supersymmetrization.
} 
The equations of motion are derived as

$$
\dot{X}^{i}=-i\left[X^{i}, V\right]=\frac{1}{I} \epsilon^{i j k} X_{j} E_{k},
$$

where $E_{i}=-\partial_{i} V$. (233) indicates a hyperbolic version of the cyclotron motion of the center of mass coordinates $X^{i}$. The Hall law

$$
E^{i} \dot{X}_{i}=0
$$

and the cyclotron motion

$$
F_{i j} \dot{X}^{j}=-\left(\eta_{i j}-X_{i} X_{j}\right) E^{j},
$$

follow from (233). Here, $F_{i j}$ is the field strength of the monopole:

$$
F_{i j}=\partial_{i} A_{j}-\partial_{j} A_{i}=-\frac{I}{2} \epsilon_{i i k} x^{k} .
$$

\subsection{Lowest Landau level on four-hyperboloid}

Eq.(153) suggests two superficially different expressions for $H^{4,3}$ :

$$
H^{4,3} \sim H^{2,2} \otimes H^{2,1} \sim \mathbb{C} P^{1,2} \otimes S^{1} .
$$

In either case, the bundles are principal bundles :

$$
H^{2,1} \simeq S U(1,1), \quad S^{1} \simeq U(1) .
$$

This observation implies that $H^{2,2}$ in $S U(1,1)$ monopole background is equivalent to $\mathbb{C} P^{1,2}$ in $U(1)$ monopole background. Here, we demonstrate this speculation in the context of lowest Landau level physics. The following analysis is a non-compact extension of the analysis in Ref.[84].

\subsubsection{Lowest Landau level on $H^{2,2}$ in $S U(1,1)$ monopole background}

In $S U(1,1)$ monopole background, one-particle Lagrangian is given by

$$
L=\frac{M}{2} \eta_{a b} \dot{x}^{a} \dot{x}^{b}+\dot{x}^{a} A_{a}
$$

where $x^{a}(a=1,2,3,4,5)$ are coordinates on $H^{2,2}$ subject to

$$
\eta_{a b} x^{a} x^{b}=1
$$

with $S O(3,2)$ metric $\eta_{a b}=\operatorname{diag}(-,-,+,+,+)$, and $A_{a}$ represents the $S U(1,1)$ monopole gauge field $(209)^{29}$. The Lagrangian (239) apparently respects the $S O(3,2)$ symmetry. Meanwhile in the lowest Landau level, the mass term drops and the gauge interaction term only survives to yield

$$
L_{L L L}=\dot{x}^{a} A_{a}=-i I \psi^{\dagger} k \frac{d \psi}{d t},
$$

\footnotetext{
${ }^{29}$ The corresponding Landau problem is investigated in Ref.[32]. The Landau problem on $H^{4,0}$ in $S U(2)$ monopole background (which corresponds to the hybrid 2nd Hopf map) is also argued in Ref.[85].
} 
with the constraint for the $S O(3,2)$ Hopf spinor

$$
\psi^{\dagger} k \psi=1 \text {. }
$$

One may see that both (241) and (242) enjoy the enhanced $S U(2,2) \simeq S O(4,2)$ symmetry as the rotational symmetry of $\psi$. From the lowest Landau level Lagrangian (241), the canonical conjugate of $\psi$ is derived as

$$
\pi=-i I \psi^{\dagger} k
$$

With the canonical quantization

$$
\left[\psi_{\alpha}, \pi_{\beta}\right]=i \delta_{\alpha \beta}
$$

we have

$$
\psi^{*}=\frac{1}{I} k \frac{\partial}{\partial \psi} .
$$

In quantum mechanics, the normalization (242) is transformed to a constraint on the Hilbert space

$$
\psi^{t} \frac{\partial}{\partial \psi} f_{L L L}=I f_{L L L}
$$

Then, the lowest Landau level bases are given by the homogeneous polynomials of the components of the $S O(3,2)$ Hopf spinor. Furthermore, $x^{a}(92)$ reduce to

$$
X_{a}=\frac{1}{I} \psi^{t} \gamma_{a}^{t} \frac{\partial}{\partial \psi}
$$

Also, the total angular momentum $L_{a b}$ reduces to the field strength, $L_{a b} \rightarrow-F_{a b}$, and hence the non-commutative relations

$$
\left[X_{a}, X_{b}\right]=4 i L_{a b}
$$

are rewritten as

$$
\left[X_{a}, X_{b}\right]=-4 i F_{a b} .
$$

Consequently, in the lowest Landau level, we derive the equations of motion as

$$
\dot{X}_{a}=-i\left[X_{a}, V\right]=4 F_{a b} E^{b},
$$

where $E_{a}=-\partial_{a} V$. From the equations of motion (250), we obtain the Hall law

$$
E^{a} \dot{X}_{a}=0
$$

and a generalized cyclotron motion

$$
F^{a b} \dot{X}_{b}=-\left(\eta^{a b}-x^{a} x^{b}\right) E_{b} \cdot\left(\tau_{i}\right)^{2}-i F^{a b} E_{b},
$$

where (212) was used. 


\subsection{2 $U(1)$ monopole gauge fields on $\mathbb{C} P^{1,2}$}

The coordinates on $H^{4,3}$ are parameterized as (207) with (208) and the $H^{2,1}$-fibre is represented as

$$
\phi=\frac{1}{\sqrt{2\left(1+n^{3}\right)}}\left(\begin{array}{c}
1+n^{3} \\
n^{1}+i n^{2}
\end{array}\right) e^{i \chi}
$$

with

$$
\eta_{i j} n^{i} n^{j}=-n^{1} n^{1}-n^{2} n^{2}+n^{3} n^{3}=1 .
$$

The $U(1)$ gauge field on $\mathbb{C} P^{1,2}$ is derived as

$$
\mathcal{A}=-i \psi^{\dagger} k d \psi=\left.d x^{a} \mathcal{A}_{a}(x, n)\right|_{I=1}+\left.d n^{i} \mathcal{A}_{i}(n)\right|_{I=1},
$$

where $\mathcal{A}_{a}(a=1,2,3,4,5)$ and $\mathcal{A}_{i}(i=1,2,3)$ are respectively given by

$$
\begin{aligned}
& \mathcal{A}_{\mu}(x, n)=\frac{I}{2} \eta_{\mu \nu i}^{\prime} \frac{x^{\nu}}{1+x^{5}} n^{i}, \quad \mathcal{A}_{5}=0, \\
& \mathcal{A}_{i}(n)=\frac{I}{2} \epsilon_{i j 3} \frac{n^{j}}{1+n^{3}} .
\end{aligned}
$$

Here, $\eta_{\mu \nu i}^{\prime}$ denotes the split-'t Hooft tensor (210) and $I / 2$ ( $I$ : integer) stands for $U(1)$ monopole charge. $\mathcal{A}_{a}$ and $\mathcal{A}_{i}$ are tangent to the surface of $H^{2,2}$ and $H^{2,0}$, respectively:

$$
\eta_{a b} \mathcal{A}_{a} x^{b}=\eta_{i j} \mathcal{A}^{i} n^{j}=0
$$

The $U(1)$ field strength

$$
\mathcal{F}_{a b}=\partial_{a} \mathcal{A}_{b}-\partial_{b} \mathcal{A}_{a}, \quad \mathcal{F}_{a i}=\partial_{a} \mathcal{A}_{i}-\partial_{i} \mathcal{A}_{a}, \quad \mathcal{F}_{i j}=\partial_{i} \mathcal{A}_{j}-\partial_{j} \mathcal{A}_{i},
$$

is calculated as

$$
\begin{aligned}
& \mathcal{F}_{\mu \nu}=-\frac{2+x^{5}}{1+x^{5}}\left(x_{\mu} \mathcal{A}_{\nu}-x_{\nu} \mathcal{A}_{\mu}\right)-I \eta_{\mu \nu i}^{\prime} \frac{n^{i}}{1+x^{5}}, \quad \mathcal{F}_{\mu 5}=\left(1+x^{5}\right) \mathcal{A}_{\mu}, \\
& \mathcal{F}_{\mu i}=-\mathcal{F}_{i \mu}=-\frac{I}{2} \eta_{\mu \nu i}^{\prime} \frac{x^{\nu}}{1+x^{5}}-\mathcal{A}_{\mu} n_{i}, \quad \mathcal{F}_{5 i}=-\mathcal{F}_{i 5}=0, \\
& \mathcal{F}_{i j}=-\frac{I}{2} \epsilon_{i j k} n^{k}
\end{aligned}
$$

which is orthogonal to the surface of $\mathbb{C} P^{1,2}$ in the following sense:

$$
\begin{aligned}
& \eta_{a b} x^{a} \mathcal{F}^{b c}=\eta_{a b} x^{a} \mathcal{F}^{b i}=0, \\
& \eta_{i j} n^{i} \mathcal{F}^{j k}=\eta_{i j} n^{i} \mathcal{F}^{j a}=0 .
\end{aligned}
$$

Furthermore, they satisfy

$$
\begin{aligned}
& \mathcal{F}_{i j} \mathcal{F}^{j k}=-\frac{I^{2}}{4}\left(\delta_{i}{ }^{k}-n_{i} n^{k}\right) \\
& \mathcal{F}^{i j} \mathcal{F}_{j a}=-\frac{I}{2} \epsilon^{i j k} \mathcal{A}_{a j} n_{k} \\
& \mathcal{F}_{\mu i} \mathcal{F}^{i j} \mathcal{F}_{j \nu}=\frac{I^{2}}{4} \frac{1}{1+x^{5}}\left(x_{\mu} \mathcal{A}_{\nu}-x_{\nu} \mathcal{A}_{\mu}\right)+\frac{I^{3}}{8} \frac{1-x^{5}}{1+x^{5}} \eta_{\mu \nu i}^{\prime} n^{i}
\end{aligned}
$$


where $\mathcal{A}_{a i}$ in (261b) is defined as $\mathcal{A}_{a} \equiv \mathcal{A}_{a i} n^{i}$ (256a), i.e. $\mathcal{A}_{\mu i}=\frac{I}{2} \eta_{\mu \nu i}^{\prime} \frac{x^{\nu}}{1+x^{5}}, \mathcal{A}_{5 i}=0$, and the properties of the split- 't Hooft tensor, $\epsilon^{i j}{ }_{k} \eta_{\mu \nu i}^{\prime} \eta_{\rho \sigma j}^{\prime}=\eta_{\mu \rho} \eta_{\nu \sigma k}^{\prime}-\eta_{\mu \sigma} \eta_{\nu \rho k}^{\prime}-\eta_{\nu \rho} \eta_{\mu \sigma k}^{\prime}+\eta_{\nu \sigma} \eta_{\mu \rho k}^{\prime}$, was used to derive (261c). It should be noted that the right-hand side of (211) is "equal" to that of (261c) by replacing the $S U(1,1)$ matrix $\tau^{i}$ with its corresponding $c$-number $n^{i}$. Hence, we have the correspondence ${ }^{30}$ :

$$
\sigma^{3}\left[A_{a}, A_{b}\right] \leftrightarrow i \frac{4}{I^{2}} \mathcal{F}_{a i} \mathcal{F}^{i j} \mathcal{F}_{j b}
$$

Eventually, one may find that the $S U(1,1)$ and $U(1)$ field strengths are related as

$$
\sigma^{3} F_{a b} \quad \leftrightarrow \quad \mathcal{F}_{a b}+\frac{4}{I^{2}} \mathcal{F}_{a i} \mathcal{F}^{i j} \mathcal{F}_{j b}
$$

\subsubsection{Lowest Landau level on $\mathbb{C} P^{1,2}$ in $U(1)$ monopole background}

We consider the one-particle motion on $\mathbb{C} P^{1,2}$ in $U(1)$ monopole background. The one-particle Lagrangian is given by

$$
S=\int d t\left[\frac{M}{2}\left(\frac{d x^{a}}{d t}\right)^{2}+\frac{M}{2}\left(\frac{d n^{i}}{d t}\right)^{2}+\mathcal{A}_{a}(x, n) \frac{d x^{a}}{d t}+\mathcal{A}_{i}(n) \frac{d n^{i}}{d t}-V(x, n)\right] .
$$

The Lagrange multipliers, $\lambda_{1}$ and $\lambda_{2}$, are introduced to incorporate the conditions (254) and (93). With the Lagrange multipliers, the equations of motion are derived as

$$
\begin{aligned}
& M \ddot{x}_{a}=-\dot{x}^{b} \mathcal{F}_{b a}-\dot{n}^{i} \mathcal{F}_{i a}+E_{a}+2 \lambda_{1} x_{a}, \\
& M \ddot{n}_{i}=-\dot{n}^{j} \mathcal{F}_{j i}-\dot{x}^{a} \mathcal{F}_{a i}+E_{i}+2 \lambda_{2} n_{i},
\end{aligned}
$$

where $E_{a}=-\partial_{a} V, E_{i}=-\partial_{i} V$. With (254) and (93), the Lagrange multipliers are obtained as

$$
\lambda_{1}=\frac{M}{2} x^{a} \ddot{x}_{a}-\frac{1}{2} x^{a} E_{a}, \quad \lambda_{2}=\frac{M}{2} n^{i} \ddot{n}_{i}-\frac{1}{2} n^{i} E_{i} .
$$

Substituting (266) to (265), we have

$$
\begin{aligned}
& M\left(\eta_{a b}-x_{a} x_{b}\right) \ddot{x}^{b}=\mathcal{F}_{a b} \dot{x}^{b}+\mathcal{F}_{a i} \dot{n}^{i}+\left(\eta_{a b}-x_{a} x_{b}\right) E^{b}, \\
& M\left(\eta_{i j}-n_{i} n_{j}\right) \ddot{n}^{j}=\mathcal{F}_{i j} \dot{n}^{j}+\mathcal{F}_{i a} \dot{x}^{a}+\left(\eta_{i j}-n_{i} n_{j}\right) E^{j} .
\end{aligned}
$$

By multiplying $\dot{x}^{a}$ and $\dot{n}^{i}$ to (267a) and (267b) respectively, their sum yields

$$
M\left(\eta_{a b} \dot{x}^{a} \ddot{x}^{b}+\eta_{i j} \dot{n}^{i} \ddot{n}^{j}\right)=\dot{x}_{a} E^{a}+\dot{n}_{i} E^{i},
$$

where the "normalization" conditions (240) and (254) were used. In the lowest Landau level $(M \rightarrow 0),(268)$ reduces to a generalized Hall law on $\mathbb{C} P^{1,2}$ :

$$
\dot{x}_{a} E^{a}+\dot{n}_{i} E^{i}=0,
$$

\footnotetext{
${ }^{30}$ For the case $(a, b)=(\mu, 5)$ or $(a, b)=(5, \nu)$, the validity of $(262)$ is apparent since $A_{5}=0$ and $\mathcal{F}_{5 i}=0$.
} 
and (267) also reduces to

$$
\begin{aligned}
& 0=\mathcal{F}_{a b} \dot{x}^{b}+\mathcal{F}_{a i} \dot{n}^{i}+\left(\eta_{a b}-x_{a} x_{b}\right) E^{b} \\
& 0=\mathcal{F}_{i j} \dot{n}^{j}+\mathcal{F}_{i a} \dot{x}^{a}+\left(\eta_{i j}-n_{i} n_{j}\right) E^{j}
\end{aligned}
$$

We arrange these first derivative equations to derive the equation of motion for $x^{a}$. From (270b), one finds that $\dot{n}^{i}$ are related to $\dot{x}^{a}$ as

$$
\dot{n}^{i}=\frac{4}{I^{2}} \mathcal{F}^{i j} E_{j}+\frac{4}{I^{2}} \mathcal{F}^{i j} \mathcal{F}_{j a} \dot{x}^{a},
$$

where (260b) and (261a) were used. From (259c) and (261b), (271) can be rewritten as

$$
\dot{n}^{i}=-\frac{2}{I} \epsilon^{i j k}\left(E_{j}+\mathcal{A}_{a j} \dot{x}^{a}\right) n_{k}
$$

which is a natural generalization of the cyclotron motion (233). By inserting (271) to (270a), we eventually obtain the first derivative equation only for $x^{a}$ :

$$
\left(\mathcal{F}_{a b}+\frac{4}{I^{2}} \mathcal{F}_{a i} \mathcal{F}^{i j} \mathcal{F}_{j b}\right) \dot{x}^{b}=-\frac{4}{I^{2}} \mathcal{F}_{a i} \mathcal{F}^{i j} E_{j}-\left(\eta_{a b}-x_{a} x_{b}\right) E^{b} .
$$

Unlike the two-hyperboloid (235), (273) contains higher orders of $U(1)$ field strengths. When $E_{i}=0,(273)$ reduces to

$$
\left(\mathcal{F}_{a b}+\frac{4}{I^{2}} \mathcal{F}_{a i} \mathcal{F}^{i j} \mathcal{F}_{j b}\right) \dot{x}^{b}=-\left(\eta_{a b}-x_{a} x_{b}\right) E^{b} .
$$

With use of the correspondence (263), one may find equivalence between the $S U(1,1)$ case $(252)$ and the $U(1)$ case (274). Thus in the lowest Landau level, one-particle mechanics on $H^{2,2}$ in the $S U(1,1)$ monopole background and that on $\mathbb{C} P^{1,2}$ in $U(1)$ monopole background are equivalent at the level of classical equations of motion. In this way, we confirmed equivalence between fuzzy $H^{2,2}$ and fuzzy $\mathbb{C} P^{1,2}$ in the context of lowest Landau level.

\section{$7 \quad$ Summary and Discussions}

We developed a systematic construction of fuzzy ultra-hyperboloids based on gamma matrices of indefinite orthogonal groups. With the cousins of quaternions, the split and hybrid Hopf maps were introduced. We realized fuzzy two- and four-hyperboloids as the Schwinger operator version of such non-compact Hopf maps. We also performed a study of fuzzy hyperboloids in higher dimensional space-times with use of indefinite gamma matrices. There are two ways to describe fuzzy hyperboloids; one is to utilize non-unitary finite dimensional representation, while the other is to utilize unitary infinite dimensional representation. With the appropriate choice of vacuum of Schwinger operators, we showed that the Schwinger operator formalism yields infinite dimensional representation of the discrete series of non-compact groups. The geometry of fuzzy ultra-hyperboloids reflects the generalized structures of the split and hybrid Hopf maps. We illuminated such generalized enhanced symmetry and dimensional hierarchy in the geometry of fuzzy ultra-hyperboloids. Non-compact monopole gauge field is naturally induced as connection 
of the fibration of fuzzy hyperboloid. We also argued the identification between the fuzzy fourhyperboloid and the six-dimensional fuzzy indefinite complex projective space in the context of the lowest Landau level physics. We believe that the present study may be useful not only for fuzzy physics itself but also for further understanding of brane geometry, twistors, and higher spin theory.

Finally, we mention the limitation of the present work. Irreducible representation of noncompact group generally contains discrete and principal series. We focused on the discrete series and the corresponding fuzzy hyperboloids of the type $H_{F}^{\text {even,even }}$. The fuzzy hyperboloids by the principal series are of the type $H_{F}^{\text {odd,odd }}$, which includes for instance, $H_{F}^{1,1}\left(=d S_{F}^{2}=A d S_{F}^{2}\right)$ and $H_{F}^{1,3}\left(=d S_{F}^{4}\right)$. Such fuzzy hyperboloids are beyond the scope of the present study ${ }^{31}$, and systematic construction of such fuzzy hyperboloids should be addressed in future works.

\section{Acknowledgments}

I would like to thank Koichi Murakami and Satoshi Watamura for helpful discussions. I am also grateful to Taichiro Kugo for telling a useful reference in his homepage. This work was supported in part by a Grant-in-Aid for Scientific Research from the Ministry of Education, Science, Sports and Culture of Japan (Grant No.23740212).

\section{A Hybrid 3rd Hopf map}

The 1st and 2nd Hopf maps were realized by sandwiching the Pauli and $S O(5)$ gamma matrices by Hopf spinors. One may expect that such realization can be readily applied to the 3rd Hopf map. However, it is not so straightforward, since the octonions cannot be realized by matrices due to their non-associative property. Instead of using the octonions themselves, the octonion

\footnotetext{
${ }^{31}$ Still, we partially discuss a construction of fuzzy $H^{\text {odd,odd }}$ in Appendix B with use of the split-complex number.
} 
structure constants are utilized to derive the following $8 \times 8$ matrices[19, 86]

$$
\begin{aligned}
& \lambda^{1}=-i\left(\begin{array}{cccc}
\sigma_{2} & 0 & 0 & 0 \\
0 & \sigma_{2} & 0 & 0 \\
0 & 0 & \sigma_{2} & 0 \\
0 & 0 & 0 & -\sigma_{2}
\end{array}\right), \quad \lambda^{2}=\left(\begin{array}{cccc}
0 & -\sigma_{3} & 0 & 0 \\
\sigma_{3} & 0 & 0 & 0 \\
0 & 0 & 0 & -1_{2} \\
0 & 0 & 1_{2} & 0
\end{array}\right) \text {, } \\
& \lambda^{3}=\left(\begin{array}{cccc}
0 & -\sigma_{1} & 0 & 0 \\
\sigma_{1} & 0 & 0 & 0 \\
0 & 0 & 0 & -i \sigma_{2} \\
0 & 0 & -i \sigma_{2} & 0
\end{array}\right), \quad \lambda^{4}=\left(\begin{array}{cccc}
0 & 0 & -\sigma_{3} & 0 \\
0 & 0 & 0 & 1_{2} \\
\sigma_{3} & 0 & 0 & 0 \\
0 & -1_{2} & 0 & 0
\end{array}\right) \text {, } \\
& \lambda^{5}=\left(\begin{array}{cccc}
0 & 0 & -\sigma_{1} & 0 \\
0 & 0 & 0 & i \sigma_{2} \\
\sigma_{1} & 0 & 0 & 0 \\
0 & i \sigma_{2} & 0 & 0
\end{array}\right), \quad \lambda^{6}=\left(\begin{array}{cccc}
0 & 0 & 0 & -1_{2} \\
0 & 0 & -\sigma_{3} & 0 \\
0 & \sigma_{3} & 0 & 0 \\
1_{2} & 0 & 0 & 0
\end{array}\right), \\
& \lambda^{7}=\left(\begin{array}{cccc}
0 & 0 & 0 & -i \sigma_{2} \\
0 & 0 & -\sigma_{1} & 0 \\
0 & \sigma_{1} & 0 & 0 \\
-i \sigma_{2} & 0 & 0 & 0
\end{array}\right)
\end{aligned}
$$

They are real antisymmetric matrices that satisfy

$$
\left\{\lambda^{I}, \lambda^{J}\right\}=-2 \delta^{I J}
$$

With $\lambda^{0} \equiv 1_{8}, \lambda^{0}$ and $\lambda^{I}(I=1,2, \cdots, 7)$ are regarded as the $S O(8)$ "Weyl + " gamma matrices. From $\lambda^{0}$ and $\lambda^{I}$, the $S O(1,8)$ gamma matrices $\Gamma^{A}$ are constructed as

$$
\Gamma^{I}=\lambda^{I} \otimes \sigma^{1}, \quad \Gamma^{8}=i 1_{8} \otimes \sigma^{2}, \quad \Gamma^{9}=1_{8} \otimes \sigma^{3},
$$

or

$$
\Gamma^{I}=\left(\begin{array}{cc}
0 & \lambda^{I} \\
\lambda^{I} & 0
\end{array}\right), \quad \Gamma^{8}=\left(\begin{array}{cc}
0 & 1_{8} \\
-1_{8} & 0
\end{array}\right), \quad \Gamma^{9}=\left(\begin{array}{cc}
1_{8} & 0 \\
0 & -1_{8}
\end{array}\right)
$$

which satisfy

$$
\left\{\Gamma^{A}, \Gamma^{B}\right\}=2 \eta^{A B}
$$

where $A, B=1,2, \cdots, 9$ and $\eta_{A B}=\eta^{A B}=\operatorname{diag}(-,-,-,-,-,-,-,-,+) . \Gamma^{I}$ and $\Gamma^{8}$ are real antisymmetric matrices:

$$
\left(\Gamma^{I}\right)^{t}=\Gamma_{I}=-\Gamma^{I}, \quad\left(\Gamma^{8}\right)^{t}=\Gamma_{8}=-\Gamma^{8} .
$$

Since $\Gamma_{A}$ are real matrices, the $S O(1,8)$ generators, $\Sigma_{A B}=-i \frac{1}{4}\left[\Gamma_{A}, \Gamma_{B}\right]$, are purely imaginary: $\Sigma_{A B}^{*}=-\Sigma_{A B}$. Thus, the present representation is Majorana representation, in which the charge conjugation matrix is given by an unit matrix, and the $S O(1,8)$ Majorana spinor is simply represented by (16-component) real spinor. From (50), the hermitianizing matrix $K$ is constructed as

$$
K=\Gamma^{1} \Gamma^{2} \cdots \Gamma^{8}=\Gamma^{9}=\left(\begin{array}{cc}
1_{8} & 0 \\
0 & -1_{8}
\end{array}\right)
$$


and the gamma matrices are hermitianized as

$$
K^{A}=K \Gamma^{A} .
$$

In detail,

$$
K^{I}=\left(\begin{array}{cc}
0 & \lambda^{I} \\
-\lambda^{I} & 0
\end{array}\right), \quad K^{8}=\left(\begin{array}{cc}
0 & 1_{8} \\
1_{8} & 0
\end{array}\right), \quad K^{9}=\left(\begin{array}{cc}
1_{8} & 0 \\
0 & 1_{8}
\end{array}\right)
$$

The $S O(1,8)$ Hopf spinor is an $S O(1,8)$ Majorana spinor ${ }^{32}$ subject to the normalization condition

$$
\Psi^{t} K \Psi=\Psi_{1}^{2}+\Psi_{2}^{2}+\cdots+\Psi_{8}^{2}-\Psi_{9}^{2}-\Psi_{10}^{2}-\cdots-\Psi_{16}^{2}=1,
$$

and hence $\Psi$ is regarded as coordinates on $H^{8,7}$. By sandwiching $\Gamma_{A}$ between the 3 rd Hopf spinors, we realize the hybrid 3rd Hopf map, $H^{8,7} \stackrel{S^{7}}{\longrightarrow} H^{8,0}$, as

$$
\Psi \rightarrow x^{A}=\Psi^{t} K^{A} \Psi .
$$

Here, $x_{A}$ are coordinates on $H^{8,0}$, since

$$
\sum_{A, B=1,2, \cdots, 9} \eta_{A B} x^{A} x^{B}=\left(\Psi^{t} K \Psi\right)^{2}=1 .
$$

The $S O(1,8)$ Hopf spinor $\Psi$ is represented as

$$
\Psi=\frac{1}{\sqrt{2\left(1+x^{9}\right)}}\left(\begin{array}{c}
\left(1+x^{9}\right) \Phi \\
\left(x^{8}-\lambda^{I} x^{I}\right) \Phi
\end{array}\right),
$$

where $\Phi$ is an $S O(7)$ real 8-component spinor subject to the constraint

$$
\Phi^{t} \Phi=1
$$

representing the $S^{7}$-bundle. The connection of $S^{7}$-bundle is evaluated as

$$
A=-i \Psi^{t} K d \Psi=-\frac{1}{2\left(1+x^{9}\right)} \sigma_{M N} x^{N} d x^{M}
$$

where

$$
\sigma_{I J}=-i \frac{1}{4}\left[\lambda_{I}, \lambda_{J}\right], \quad \sigma_{I 8}=-\sigma_{8 I}=i \frac{1}{2} \lambda_{I},
$$

with $\lambda_{I} \equiv-\lambda^{I}$. These represent the $S O(8)$ monopole gauge field on $H^{8,0}$. The corresponding field strength

$$
F_{A B}=\partial_{A} A_{B}-\partial_{B} A_{A}-i\left[A_{A}, A_{B}\right]
$$

is derived as

$$
F_{M N}=x_{M} A_{N}-x_{N} A_{M}-\sigma_{M N}, \quad F_{M 9}=-F_{9 M}=\left(1+x^{9}\right) A_{M} .
$$

\footnotetext{
${ }^{32}$ The $S O(p, q)$ with $p+q=9$ accommodate Majorana spinor only when $(p, q)=(9,0),(5,4)$ and $(1,8)$. The former two cases correspond to the compact and split 3rd Hopf maps, and the last corresponds to the hybrid 3rd Hopf map.
} 


\section{B Split Algebra and Fuzzy Split-Hyperboloid}

The split-imaginary unit $j$ is introduced so as to satisfy

$$
j^{2}=1, \quad j^{*}=-j,
$$

where $*$ denotes complex conjugation. With two real numbers $x$ and $y$, the split-complex number is defined as

$$
z=x+j y
$$

Its complex conjugation is given by

$$
z^{*}=x-j y
$$

and then

$$
z^{*} z=z z^{*}=x^{2}-y^{2}
$$

The split Hopf maps are naturally introduced by adopting the split-complex number [45]. Similarly, the unitary and special unitary groups of split-complex numbers are introduced as

$$
\begin{aligned}
& \mathcal{S U}(p) \equiv S U\left(p ; \mathbb{C}^{\prime}\right)=S L(p, \mathbb{R}) \\
& \mathcal{U}(p) \equiv U\left(p ; \mathbb{C}^{\prime}\right)=\mathcal{U}(1) \otimes \mathcal{S U}(p)
\end{aligned}
$$

Note that the (quasi-)split hyperboloid $H^{p+1, p}$ is represented by the coset

$$
H^{p+1, p} \simeq \mathcal{S U}(p+1) / \mathcal{S U}(p) .
$$

\section{B.1 Fuzzy two-hyperboloid: $\mathcal{H}_{F}^{1,1}$}

The coordinates on $S_{F}^{2}$ satisfy the $S U(2)$ algebra and square of the radius of $S_{F}^{2}$ is specified by the eigenvalues of the $S U(2)$ Casimir. In a similar manner, we introduce fuzzy split-hyperboloid, $\mathcal{H}_{F}^{1,1}$, based on the split imaginary unit. The coordinates on $\mathcal{H}_{F}^{1,1}$ are constructed as

$$
X^{i}=\Phi^{\dagger} \sigma^{i} \Phi
$$

where $\sigma^{i}(i=1,2,3)$ are "Pauli matrices" with split imaginary unit:

$$
\sigma^{1}=\left(\begin{array}{cc}
1 & 0 \\
0 & -1
\end{array}\right), \quad \sigma^{2}=\left(\begin{array}{ll}
0 & 1 \\
1 & 0
\end{array}\right), \quad \sigma^{3}=\left(\begin{array}{cc}
0 & -j \\
j & 0
\end{array}\right),
$$

and $\Phi$ denotes a two-component Schwinger operator whose components satisfy

$$
\left[\Phi_{\alpha}, \Phi_{\beta}^{\dagger}\right]=\delta_{\alpha \beta}
$$

(300) gives $S O(2,1)$ gamma matrices in the sense that the "Pauli matrices" satisfy

$$
\left\{\sigma^{i}, \sigma^{j}\right\}=2 \eta^{i j}
$$

with $\eta^{i j}=\operatorname{diag}(+,+,-)$, and then $X^{i}(299)$ satisfy the $S U(1,1)$ algebra

$$
\left[X^{i}, X^{j}\right]=j \epsilon^{i j k} X_{k}
$$


Square of the radius of $\mathcal{H}_{F}^{1,1}$ is given by the $S U(1,1)$ Casimir

$$
\eta_{i j} X^{i} X^{j}=X^{2}+Y^{2}-Z^{2}=\left(\Phi^{\dagger} \Phi\right)\left(\Phi^{\dagger} \Phi+2\right) .
$$

Note that the right-hand side of (304) is invariant under " $S U(1,1)$ rotations" generated by $(300)$. Thus, the spectra of square of the radius of $H_{F}^{1,1}$ are given by $l(l+1)$ with $l=0,1 / 2,1,3 / 2, \cdots$. The commutative counterpart of $H_{F}^{1,1}$ is one-leaf hyperboloid

$$
H^{1,1} \simeq S U(1,1) / S O(1,1) \simeq A d S^{2} \simeq \mathbb{C}^{\prime} P^{1} .
$$

$\mathbb{C}^{\prime} P^{1}$ will be introduced in Appendix C.2.

\section{B.2 Fuzzy four-hyperboloid: $\mathcal{H}_{F}^{2,2}$}

Next, we discuss the fuzzy four-hyperboloid based on the split-imaginary unit. With the Pauli matrices $\sigma^{i}$ made of the split-imaginary unit $(300)$, " $S O(3,2)$ " gamma matrices are constructed as

$$
\gamma^{i}=\left(\begin{array}{cc}
0 & j \sigma^{i} \\
-j \sigma^{i} & 0
\end{array}\right), \quad \gamma^{4}=\left(\begin{array}{cc}
0 & 1_{2} \\
1_{2} & 0
\end{array}\right), \quad \gamma^{i}=\left(\begin{array}{cc}
1_{2} & 0 \\
0 & -1_{2}
\end{array}\right)
$$

which satisfy

$$
\left\{\gamma^{a}, \gamma^{b}\right\}=2 \eta^{a b}
$$

where $\eta_{a b}=(-,-,+,+,+)$. The "SO(3,2) generators" are also given by

$$
\sigma^{a b}=-j \frac{1}{4}\left[\gamma^{a}, \gamma^{b}\right]
$$

which satisfy

$$
\begin{aligned}
& {\left[\gamma_{a}, \sigma_{b c}\right]=-j\left(\eta_{a b} \gamma_{c}-\eta_{a c} \gamma_{b}\right),} \\
& {\left[\sigma_{a b}, \sigma_{c d}\right]=j\left(\eta_{a c} \sigma_{b d}-\eta_{a d} \sigma_{b c}+\eta_{b d} \sigma_{a c}-\eta_{b c} \sigma_{a d}\right) .}
\end{aligned}
$$

With four-component Schwinger operator $\Phi,\left[\Phi_{\alpha}, \Phi_{\beta}^{\dagger}\right]=\delta_{\alpha \beta}(\alpha, \beta=1,2,3,4)$, we introduce the fuzzy coordinates on $\mathcal{H}_{F}^{2,2}$ as

$$
X^{a}=\Phi^{\dagger} \gamma^{a} \Phi
$$

and square of the radius of $\mathcal{H}_{F}^{2,2}$ is derived as

$$
\eta_{a b} X^{a} X^{b}=\left(\Phi^{\dagger} \Phi\right)\left(\Phi^{\dagger} \Phi+4\right) .
$$

With $X_{A B}(A, B=1,2,3,4,5,6) ; X_{a 6} \equiv-\frac{1}{2} X_{a}$ and $X_{a b} \equiv-i \frac{1}{4}\left[X_{a}, X_{b}\right], X_{A B}$ satisfy the closed algebra:

$$
\left[X_{A B}, X_{C D}\right]=j\left(\eta_{A C} X_{B D}-\eta_{A D} X_{B C}+\eta_{B D} X_{A C}-\eta_{B C} X_{A D}\right),
$$

where $\eta_{A B}=\operatorname{diag}(-,-,+,+,+,-)$, i.e. the $S O(3,3)$ metric. (Remember, in the case of $H_{F}^{2,2}$, $X_{A B}$ satisfy the $S O(4,2)$ algebra (146).) Note that $S O(3,3)$ is isomorphic to the split-imaginary special unitary group: $S O(3,3) \simeq \mathcal{S U}(4)$. Then, $\mathcal{H}_{F}^{2,2}$ is represented as the coset

$$
\mathcal{H}_{F}^{2,2} \simeq S O(3,3) / \mathcal{U}(3) \simeq \mathcal{S U}(4) / \mathcal{U}(3) \simeq \mathbb{C}^{\prime} P^{3},
$$


where $\mathbb{C}^{\prime} P^{3}$ denotes split-complex projective space (see Appendix C.2). With the original symmetry $S O(3,2), \mathcal{H}_{F}^{2,2}$ can be expressed as

$$
\mathcal{H}_{F}^{2,2} \simeq S O(3,2) / \mathcal{U}(2)
$$

since $\mathbb{C}^{\prime} P^{3} \sim H^{2,2} \otimes H^{1,1} \simeq S O(3,2) / S O(2,2) \otimes S O(2,1) / S O(1,1) \simeq S O(3,2) /(S O(2,1) \otimes$ $S O(1,1))^{33}$. This result is a natural split-complex number version of fuzzy four-sphere: $S_{F}^{4} \simeq$ $S O(5) / U(2)$. Also note that $\mathcal{H}_{F}^{2,2}$ is different from $H_{F}^{2,2}(151)$.

\section{B.3 Fuzzy split-hyperboloids: $\mathcal{H}_{F}^{p, p}$}

From the above discussions, it may be natural to expect that fuzzy split-hyperboloids are generally given by the coset:

$$
\mathcal{H}_{F}^{p, p} \simeq S O(p+1, p) / \mathcal{U}(p)
$$

This is a natural split signature counterpart of the fuzzy sphere, $S_{F}^{2 p} \simeq S O(2 p+1) / U(p)[7] . \mathcal{H}_{F}^{p, p}$ is locally given by

$$
\mathcal{H}^{p, p} \sim H^{p, p} \otimes S O(p, p) / \mathcal{U}(p),
$$

where $H^{p, p}$ and $S O(p, p) / \mathcal{U}(p)$ respectively represent the basemanifold and the fibre on it. With

$$
S O(p, p) / S O(p, p-1) \simeq \mathcal{U}(p) / \mathcal{U}(p-1)\left(\simeq H^{p, p-1}\right)
$$

the fuzzy split-hyperboloid may be expressed as

$$
\begin{aligned}
\mathcal{H}_{F}^{p, p} & \sim H^{p, p} \otimes S O(p, p-1) / \mathcal{U}(p-1) \\
& \simeq H^{p, p} \otimes \mathcal{H}_{F}^{p-1, p-1} \\
& \sim H^{p, p} \otimes H^{p-1, p-1} \otimes H^{p-2, p-2} \otimes \cdots \otimes H^{2,2} \otimes H^{1,1} .
\end{aligned}
$$

Then in low dimensions, we have

$$
\begin{aligned}
& \mathcal{H}_{F}^{1,1} \simeq S O(2,1) / \mathcal{U}(1) \simeq H^{1,1}, \\
& \mathcal{H}_{F}^{2,2} \simeq S O(3,2) / \mathcal{U}(2) \sim H^{2,2} \otimes H^{1,1}, \\
& \mathcal{H}_{F}^{3,3} \simeq S O(4,3) / \mathcal{U}(3) \sim H^{3,3} \otimes H^{2,2} \otimes H^{1,1}, \\
& \mathcal{H}_{F}^{4,4} \simeq S O(5,4) / \mathcal{U}(4) \sim H^{4,4} \otimes H^{3,3} \otimes H^{2,2} \otimes H^{1,1} .
\end{aligned}
$$

The dimension of the fuzzy split-hyperboloid $H_{F}^{p, p}$ is given by $\sum_{k=1}^{p} 2 k=p(p+1)$.

The coordinates of $\mathcal{H}_{F}^{p, p}$ are regarded as the gamma matrices of the (quasi-)split orthogonal groups $S O(p+1, p)$. Then, the hierarchical geometry (320) can also be observed in the structure

\footnotetext{
${ }^{33}$ Here, we used

$$
S O(2,2) \simeq S U(1,1) \otimes S U(1,1), \quad S O(2,1) \simeq S U(1,1) \simeq \mathcal{S U}(2), \quad S O(1,1) \simeq \mathcal{U}(1) .
$$
}


of the $S O(p+1, p)$ gamma matrices. The $S O(p+1, p)$ gamma matrices, $\Gamma^{a}(a=1,2, \cdots, 2 p+1)$, are given by

$$
\Gamma^{i}=\left(\begin{array}{cc}
0 & j \gamma^{2 p-i} \\
-j \gamma^{2 p-i} & 0
\end{array}\right), \quad \Gamma^{2 p}=\left(\begin{array}{ll}
0 & 1 \\
1 & 0
\end{array}\right), \quad \Gamma^{2 p+1}=\left(\begin{array}{cc}
1 & 0 \\
0 & -1
\end{array}\right),
$$

where $i=1,2, \cdots, 2 p-1$. The anti-commutation relations of $\Gamma^{a}$ read as

$$
\left\{\Gamma^{a}, \Gamma^{b}\right\}=2 \xi^{a b}
$$

where $\xi^{a b}=\left(-\eta^{2 p-i, 2 p-j},+,+\right)$. Thus, from $S O(p+1, p)$ gamma matrices, we can construct $S O(p+2, p+1)$ gamma matrices. With iterative use of $(321)$, we have the gamma matrices of the following groups

$$
S O(2,1) \rightarrow S O(3,2) \rightarrow S O(4,3) \rightarrow S O(5,4) \rightarrow \cdots
$$

The split Hopf spinor for $\mathcal{H}_{F}^{p, p}$ is constructed as

$$
\Psi=M \psi,
$$

where $M$ denotes $2^{p} \times 2^{p-1}$ matrix of the form ${ }^{34}$

$$
M=\frac{1}{\sqrt{2\left(1+x^{2 p+1}\right)}}\left(\begin{array}{c}
\left(1+x^{2 p+1}\right) 1_{2^{p-1}} \\
x^{2 p} 1_{2^{p-1}}-j x^{i} \gamma_{2 p-i}
\end{array}\right),
$$

with $x^{i} \gamma_{2 p-i} \equiv \xi_{i j} x^{i} \gamma^{2 p-j}$. As found in (319), $\mathcal{H}_{F}^{p, p}$ is regarded as fuzzy fibre-bundle of the fibre $\mathcal{H}_{F}^{p-1, p-1}$ over the basemanifold $H^{2,2}$. The connection of fuzzy bundle $\mathcal{H}_{F}^{p-1, p-1}$ is derived as

$$
A=d x^{a} A_{a}=-j M^{\dagger} d M
$$

where

$$
A_{\mu}=-\frac{1}{1+x^{2 p+1}} \sigma_{\mu \nu} x^{\nu}, \quad A_{2 p+1}=0 .
$$

Here, $\sigma_{\mu \nu}(\mu, \nu=1,2, \cdots, 2 n)$ are $S O(p, p)$ generators given by

$$
\sigma_{i j}=-j \frac{1}{4}\left[\gamma_{2 p-i}, \gamma_{2 p-j}\right], \quad \sigma_{i, 2 p}=-\sigma_{2 p, i}=\frac{1}{2} \gamma_{2 p-1},
$$

with $i=1,2, \cdots, n-1$. $A_{a}$ represent $S O(p, p)$ monopole gauge field. The field strength, $F_{a b}=$ $\partial_{a} A_{b}-\partial_{b} A_{a}-j\left[A_{a}, A_{b}\right]$, is derived as

$$
F_{\mu \nu}=-x_{\mu} A_{\nu}+x_{\nu} A_{\mu}+\sigma_{\mu \nu}, \quad F_{\mu, 2 p+1}=-F_{2 p+1, \mu}=\left(1+x^{2 p+1}\right) A_{\mu} .
$$

${ }^{34} M$ satisfies

$$
\begin{aligned}
& M^{\dagger} M=\mathbf{1} \\
& M M^{\dagger}=\frac{1}{2}\left(\begin{array}{cc}
1_{2^{p-1}}+x^{2 p+1} & x^{2 p} 1_{2^{p-1}}+j x^{i} \gamma_{2 p-i} \\
x^{2 p} 1_{2^{p-1}}-j x^{i} \gamma_{2 p-i} & 1_{2^{p-1}}-x^{2 p+1}
\end{array}\right)=\frac{1}{2}\left(1+x_{a} \Gamma^{a}\right) \equiv P,
\end{aligned}
$$

where $P$ is a projection operator:

$$
P^{2}=P, \quad P \Psi=\Psi
$$




\section{Indefinite Complex Projective Spaces}

We briefly introduce indefinite complex and split-complex projective spaces.

\section{C.1 Indefinite complex projective spaces}

Indefinite complex projective space signifies complex projective space in indefinite complex space. $\mathbb{C} P^{p, q}$ is defined so as to satisfy the condition of $H^{2 q, 2 p+1}$

$$
\sum_{i=1}^{p+1} z_{i}{ }^{*} z_{i}-\sum_{j=1}^{q} z_{j}^{\prime *} z_{j}^{\prime}=1,
$$

modulo $U(1)$ :

$$
\left(z_{1}, z_{2}, \cdots, z_{p+1}, z_{1}^{\prime}, z_{2}^{\prime}, \cdots, z_{q}^{\prime}\right) \sim e^{i \theta}\left(z_{1}, z_{2}, \cdots, z_{p+1}, z_{1}^{\prime}, z_{2}^{\prime}, \cdots, z_{q}^{\prime}\right),
$$

and hence $\mathbb{C} P^{p, q}$ is expressed as the coset:

$$
\mathbb{C} P^{p, q} \simeq H^{2 q, 2 p+1} / S^{1}
$$

or

$$
\mathbb{C} P^{p, q} \simeq S U(p+1, q) / U(p, q) \simeq S U(q, p+1) / U(q, p) .
$$

(334) can be regarded as a higher dimensional generalization of the non-compact 1st Hopf map. Indeed, for $(p, q)=(0,1)$, we reproduce the non-compact 1st Hopf map,

$$
\mathbb{C} P^{0,1} \simeq S U(1,1) / U(1) \simeq H^{2,1} / S^{1} \sim H^{2,0}
$$

Also, $\mathbb{C} P^{p, q}$ are related to the 2nd and 3rd split Hopf maps:

$$
\begin{aligned}
& \mathbb{C} P^{1,2} \simeq S U(2,2) / U(1,2) \simeq H^{4,3} / S^{1} \sim H^{2,2} \otimes H^{2,0} \\
& \mathbb{C} P^{3,4} \simeq S U(4,4) / U(3,4) \simeq H^{8,7} / S^{1} \sim H^{4,4} \otimes H^{2,2} \otimes H^{2,0} .
\end{aligned}
$$

(337a) is the basic relation of the discussion in Section 6.2.

\section{C.2 Split-complex projective spaces}

The split-complex projective spaces $\mathbb{C}^{\prime} P^{p}$ are introduced by replacing the usual imaginary unit with the split imaginary unit in the definition of $\mathbb{C} P^{p}$ : With $z_{i}=x_{i}+j y_{i}(i=1,2, \cdots, p+1) \in$ $\mathbb{C}^{\prime p+1}, \mathbb{C}^{\prime} P^{p}$ is defined so as to satisfy the condition of $H^{p+1, p}$

$$
\sum_{i=1}^{p+1} z_{i}^{*} z_{i}=\sum_{i=1}^{p+1} x_{i} x_{i}-\sum_{i=1}^{p+1} y_{i} y_{i}=1
$$

modulo $\mathcal{U}(1)$

$$
\left(z_{1}, z_{2}, \cdots, z_{p+1}\right) \sim e^{j \theta}\left(z_{1}, z_{2}, \cdots, z_{p+1}\right)
$$


Therefore, $\mathbb{C}^{\prime} P^{p}$ can be expressed by the coset:

$$
\mathbb{C}^{\prime} P^{p} \simeq H^{p+1, p} / H^{1}
$$

or

$$
\mathbb{C}^{\prime} P^{p} \simeq \mathcal{S U}(p+1) / \mathcal{U}(p),
$$

where we used $H^{1} \simeq \mathcal{U}(1)$ and (298). In particular, related to the split Hopf maps, we have ${ }^{35}$

$$
\begin{aligned}
& \mathbb{C}^{\prime} P^{1} \simeq \mathcal{S U}(2) / \mathcal{U}(1) \simeq H^{2,1} / H^{1,0} \sim H^{1,1} \\
& \mathbb{C}^{\prime} P^{3} \simeq \mathcal{S U}(4) / \mathcal{U}(3) \simeq H^{4,3} / H^{1,0} \sim H^{2,2} \otimes H^{1,1} \\
& \mathbb{C}^{\prime} P^{7} \simeq \mathcal{S U}(8) / \mathcal{U}(7) \simeq H^{8,7} / H^{1,0} \sim H^{4,4} \otimes H^{2,2} \otimes H^{1,1}
\end{aligned}
$$

Notice that for split-complex projective space, we need not define its indefinite version. It is because $\mathbb{C}^{\prime} P^{p, q}$ satisfies

$$
\sum_{i=1}^{p} z_{i}^{*} z_{1}-\sum_{j=1}^{q+1} \tilde{z}_{j}^{*} \tilde{z}_{j}=\left(\sum_{i=1}^{p} x_{i} x_{i}+\sum_{j=1}^{q+1} \tilde{y}_{j} \tilde{y}_{j}\right)-\left(\sum_{j=1}^{p} y_{j} y_{j}+\sum_{i=1}^{q+1} \tilde{x}_{i} \tilde{x}_{i}\right)=1,
$$

modulo $\mathcal{U}(1)$, however with $z_{i}^{\prime} \equiv x_{i}+j y_{i}(i=1,2, \cdots, p)$ and $z_{p+i}^{\prime} \equiv \tilde{y}_{i}+j \tilde{x}_{i}(i=1,2, \cdots, q+1)$, (344) can be rewritten in the form of (338):

$$
\sum_{i=1}^{p+q+1} z_{i}^{\prime *} z_{i}^{\prime}=1
$$

This indicates ${ }^{36}$

$$
\mathbb{C}^{\prime} P^{p, q}=\mathbb{C}^{\prime} P^{p+q}
$$

\footnotetext{
${ }^{35}$ Eq.(341) is a non-compact version of the expression $\mathbb{C} P^{p} \simeq S U(p+1) / U(p) \simeq S^{2 p+1} / S^{1}$. Eq.(343) is the split-signature version of

$$
\begin{aligned}
& \mathbb{C} P^{1} \simeq S U(2) / U(1) \simeq S^{3} / S^{1} \sim S^{2} \\
& \mathbb{C} P^{3} \simeq S U(4) / U(3) \simeq S^{7} / S^{1} \sim S^{4} \otimes S^{2} \\
& \mathbb{C} P^{7} \simeq S U(8) / U(7) \simeq S^{15} / S^{1} \sim S^{8} \otimes S^{4} \otimes S^{2} .
\end{aligned}
$$

${ }^{36}(349)$ is also consistent with the cosets for $\mathbb{C}^{\prime} P^{p, q}$ and $\mathbb{C}^{\prime} P^{p+q}$ :

$$
\mathbb{C}^{\prime} P^{p, q} \simeq \mathcal{S U}(p+1, q) / \mathcal{U}(p, q)
$$

and

$$
\mathbb{C}^{\prime} P^{p+q} \simeq \mathcal{S U}(p+q+1) / \mathcal{U}(p+q)
$$

since

$$
\mathcal{S U}(p, q) \simeq \mathcal{S U}(p+q), \quad \mathcal{U}(p, q) \simeq \mathcal{U}(p+q)
$$




\section{References}

[1] F. A. Berezin, "General Concept of Quantization”, Commun.Math. Phys. 40 (1975) 153-174.

[2] Jens Hoppe, "Quantum Theory of a Massless Relativistic Surface and a Two-dimensional Bound State Problem", MIT PhD Thesis (1982).

[3] J. Madore, “The Fuzzy Sphere”, Class. Quant. Grav. 9 (1992) 69.

[4] Washington Taylor, "Lectures on D-branes, Gauge Theory and M(atrices)", hep-th/9801182.

[5] A. P. Balachandran, "Quantum Spacetimes in the Year 1", Pramana 59 (2002) 359-368; hep-th/0203259.

[6] Kazuki Hasebe, "Graded Hopf Maps and Fuzzy Superspheres", Nucl.Phys. B 853 (2011) 777827; arXiv:1106.5077.

[7] Pei-Ming Ho, Sanjaye Ramgoolam, "Higher Dimensional Geometries from Matrix Brane constructions", Nucl.Phys. B627 (2002) 266-288; hep-th/0111278.

[8] Ursula Carow-Watamura, Satoshi Watamura "Chirality and Dirac Operator on Noncommutative Sphere", Commun.Math.Phys. 183 (1997) 365-382; hep-th/9605003.

[9] Ursula Carow-Watamura, Satoshi Watamura, "Noncommutative Geometry and Gauge Theory on Fuzzy Sphere”, Commun.Math.Phys. 212 (2000) 395-413; hep-th/9801195.

[10] H. Grosse, C. Klimcik, P. Prešnajder, "On Finite 4D Quantum Field Theory in NonCommutative Geometry”, Commun.Math.Phys.180 (1996) 429-438; hep-th/9602115.

[11] Yusuke Kimura, "Noncommutative Gauge Theory on Fuzzy Four-Sphere and Matrix Model", Nucl.Phys. B637 (2002) 177-198; hep-th/0204256.

[12] Yusuke Kimura, "On Higher Dimensional Fuzzy Spherical Branes", Nucl.Phys. B664 (2003) 512-530; hep-th/0301055.

[13] Takehiro Azuma, Maxime Bagnoud, "Curved-space classical solutions of a massive supermatrix model", Nucl.Phys. B651 (2003) 71-86; hep-th/0209057.

[14] Ryuichi Nakayama, Yusuke Shimono, "Matrix Configurations for Spherical 4-branes and Noncommutative Structures on $S^{4}$ ", Nucl.Phys. B693 (2004) 176-194; hep-th/0402010.

[15] Yasuhiro Abe, “Construction of fuzzy $S^{4}$ ”, Phys.Rev. D70 (2004) 126004; hep-th/0406135.

[16] Harald Grosse, Gert Reiter, "The Fuzzy Supersphere", Journal of Geometry and Physics 28 (1998) 349-383; math-ph/9804013.

[17] A. P. Balachandran, S. Kurkcuoglu, E. Rojas, "The Star Product on the Fuzzy Supersphere", JHEP 0207 (2002) 056; hep-th/0204170. 
[18] J. Arnlind, M. Bordemann, L. Hofer, J. Hoppe, H. Shimada, "Fuzzy Riemann Surfaces", JHEP 0906 (2009) 047; hep-th/0602290.

[19] Kazuki Hasebe, "Hopf Maps, Lowest Landau Level, and Fuzzy Spheres", SIGMA 6 (2010) 071; arXiv:1009.1192.

[20] H. Grosse, C. Klimcik, P. Prešnajder, "Topologically nontrivial field configurations in noncommutative geometry", Commun.Math.Phys. 178 (1996) 507-526; hep-th/9510083.

[21] M. M. Sheikh-Jabbari, "Tiny Graviton Matrix Theory: DLCQ of IIB Plane-Wave String Theory, A Conjecture ", JHEP 0409 (2004) 017; hep-th/0406214.

[22] M. M. Sheikh-Jabbari, M. Torabian, "Classification of All 1/2 BPS Solutions of the Tiny Graviton Matrix Theory", JHEP 0504 (2005) 001; hep-th/0501001.

[23] Heinz Hopf, "Über die Abbildungen der dreidimensionalen Sphäre auf die Kugelfläche", Mathematische Annalen 104 (1931) 637-665.

[24] Heinz Hopf, "Über die Abbildungen von Sphären auf Sphären niedrigerer Dimension", Fundamenta Mathematicae 25 (1935) 427-440.

[25] Mikio Nakahara, "Geometry, Topology and Physics", (2nd ed.), IOP Publishing, (2003).

[26] John C. Baez, "The Octonions", Bull. Amer. Math. Soc. 39 (2002) 145-205. Errata in Bull Amer. Math. Soc. 42 (2005), 213; math/0105155.

[27] Kazuki Hasebe, Yusuke Kimura, "Dimensional Hierarchy in Quantum Hall Effects on Fuzzy Spheres", Phys.Lett. B602 (2004) 255-260; hep-th/0310274.

[28] Dongsu Bak, "Supersymmetric Branes in the Matrix Model of PP Wave Background", Phys.Rev. D67 (2003) 045017; hep-th/0204033.

[29] Makoto Sakaguchi, Kentaroh Yoshida, "M-theory on a Time-dependent Plane-wave", JHEP 0311 (2003) 030; hep-th/0309025.

[30] Pei-Ming Ho, Miao Li, "Fuzzy Spheres in AdS/CFT Correspondence and Holography from Noncommutativity", Nucl.Phys. B596 (2001) 259-272; hep-th/0004072.

[31] Pei-Ming Ho, Miao Li, “Large $N$ Expansion From Fuzzy AdS $S_{2}$ ”, Nucl.Phys. B590 (2000) 198-212; hep-th/0005268.

[32] Kazuki Hasebe, "Split-Quaternionic Hopf Map, Quantum Hall Effect and Twistor Theory", Phys.Rev.D81 (2010) 041702; arXiv:0902.2523.

[33] D. Mihai, G. Sparling, Ph. Tillman, "Non-Commutative Time, the Quantum Hall Effect and Twistor Theory", cond-mat/0401224.

[34] Dimitra Karabali, V.P. Nair, "Quantum Hall Effect in Higher Dimensions", Nucl.Phys. B641 (2002) 533-546; hep-th/0203264. 
[35] Jonathan J. Heckman, Herman Verlinde, "Instantons, Twistors, and Emergent Gravity", arXiv:1112.5210.

[36] M. A. Vasiliev, "Higher Spin Gauge Theories in Various Dimensions", Fortsch.Phys.52 (2004) 702-717; hep-th/0401177.

[37] M. A. Vasiliev, "Nonlinear Equations for Symmetric Massless Higher Spin Fields in $(A) d S_{d}$ ", Phys.Lett. B567 (2003) 139-151; hep-th/0304049.

[38] H. Grosse and P. Prešnajder, "The construction of noncommutative manifolds using coherent states", Lett.Math. Phys.28 (1993) 239-250.

[39] Isao Kishimoto, "Fuzzy Sphere and Hyperbolic Space from Deformation Quantization", JHEP 0103 (2001) 025; hep-th/0103018.

[40] H. Fakhri, A. Imaanpur, "Dirac Operator on Fuzzy $A d S_{2}$ ", JHEP 0303 (2003) 003; hepth/0302154.

[41] Kazuki Hasebe, "Hyperbolic Supersymmetric Quantum Hall Effect", Phys.Rev.D78 (2008) 125024; arXiv:0809.4885.

[42] Jean-Pierre Gazeau, Jihad Mourad, Julien Queva, "Fuzzy de Sitter space-times via coherent states quantization", Proceedings of the XXVIth Colloquium on Group Theoretical Methods in Physics (2006); quant-ph/0610222.

[43] Jean-Pierre Gazeau and Francesco Toppan, "A natural fuzzyness of de Sitter space-time", Class.Quant.Grav.27 (2010) 025004; arXiv:0907.0021.

[44] Joshua DeBellis, Christian Sämann, Richard J. Szabo, "Quantized Nambu-Poisson Manifolds and n-Lie Algebras", J. Math. Phys. 51 (2010) 122303; arXiv:1001.3275.

[45] Kazuki Hasebe, "The Split-Algebras and Non-compact Hopf Maps", J. Math. Phys.51 (2010) 053524; arXiv:0905.2792.

[46] Gabriel Băditoiu, "Classification of Pseudo-Riemannian submersions with totally geodesic fibres from pseudo-hyperbolic spaces", arXiv:1001.4490.

[47] J. J. Konderak, "Construction of Harmonic Maps between Pseudo-Riemannian Spheres and Hyperbolic Spaces", Proc. Am. Math. Soc. 109 (1990) 469-476.

[48] Novica Blăzić, "Paraquaternionic projective space and pseudo-Riemannian geometry", Publ. Inst. Math. 60 (1996) 101-107.

[49] R. Mosseri, R. Dandoloff, "Geometry of entangled states, Bloch spheres and Hopf fibrations", J.Phys.A34 (2001) 10243; quant-ph/0108137.

[50] Bogdan A. Bernevig, Han-Dong Chen "Geometry of the 3-Qubit State, Entanglement and Division Algebras", J. Phys. A: Math. Gen., 36 (2003) 8325; quant-ph/0302081. 
[51] Michael Rios, "Extremal Black Holes as Qudits", arXiv:1102.1193.

[52] See as a review, L. Borsten, D. Dahanayake, M. J. Duff, H. Ebrahim, W. Rubens, "Black Holes, Qubits and Octonions", Phys.Rep.471 (2009) 113-219; arXiv:0809.4685.

[53] N. Kawamoto and Y. Watabiki, "Even-dimensional generalization of Chern-Simons action and new gauge symmetry",Comm.Math.Phys. 144 (1992) 641-648.

[54] N. Kawamoto and Y. Watabiki, "Topological particle field theory, general coordinate invariance and generalized Chern-Simons actions",Comm.Math.Phys. 148 (1992) 169-176.

[55] D. C. Brody and E.-M. Graefe, "On complexified mechanics and coquaternions" J. Phys. A 44 (2011) 072001; arXiv:1012.0757.

[56] D. C. Brody and E.-M. Graefe, "Coquaternionic quantum dynamics for two-level systems", Acta Polytechnica 51 (2011) 14-20; arXiv:1105.4038.

[57] Alexander I Nesterov, F. Aceves de la Cruz "Complex magnetic monopoles, geometric phases and quantum evolution in vicinity of diabolic and exceptional points", J.Phys.A41 (2008) 485304; arXiv:0806.3720.

[58] Masatoshi Sato, Kazuki Hasebe, Kenta Esaki, Mahito Kohmoto "Time-Reversal Symmetry in Non-Hermitian Systems", Prog. Theor. Phys. 127 (2012) 937-974; arXiv:1106.1806.

[59] Kenta Esaki, Masatoshi Sato, Kazuki Hasebe, Mahito Kohmoto, "Edge states and topological phases in non-Hermitian systems", Phys. Rev. B 84 (2011) 205128; arXiv:1107.2079.

[60] Yi Chen Hu, Taylor L. Hughes "Absence of topological insulator phases in non-Hermitian PT-symmetric Hamiltonians", Phys. Rev. B 84 (2011) 153101; arXiv:1107.1064.

[61] William Rowan Hamilton, "On a new Species of Imaginary Quantities connected with a theory of Quaternions", Proceedings of the Royal Irish Academy, 2 (1844) 424-434.

[62] James Cockle, "On Certain Functions Resembling Quaternions, and on a New Imaginary Algebra", Phil. Mag. (3) 33 (1848) 435-439. "On a New Imaginary in Algebra", Phil. Mag. (3) 34 (1849) 37-47.

[63] Alexander Macfarlane, "Principles of the Algebra of Physics", Proceedings of the American Association for the Advancement of Science 40 (1892) 65-117.

[64] Shou-Cheng Zhang, Jiangping Hu "A Four Dimensional Generalization of the Quantum Hall Effect", Science 294 (2001) 823; cond-mat/0110572.

[65] Taichiro Kugo, Paul K. Townsend, "Supersymmetry and the Division Algebras", Nucl.Phys. B221 (1983) 357. See also a note about indefinite gamma matrices in Kugo's homepage, http://www2.yukawa.kyoto-u.ac.jp/ kugo.

[66] V. Bargmann, "Irreducible unitary representations of the Lorentz group", Ann. Math. 48 (1947) 568-640. 
[67] Askold Perelomov, "Generalized Coherent States and Their Applications", Springer-Verlag New York (1986).

[68] M. E. Loewe, "SO(3,2) for oscillator and hydrogenlike systems", Lecture Notes in Physics, vol. 382 (1991) 98-105.

[69] N. T. Evans, "Discrete series for the universal covering group of the 3+2 de Sitter group", J.Math. Phys, 8 (1967) 170-184.

[70] J. Dixmier, "Representations integrables du groupe de De Sitter", Bull. Soc. Math. France 89 (1961) 9-41.

[71] A. Bohm, "Generalized eigenvectors and group representations - the connection between representations of SO $(4,1)$ and the Poincare group", in Studies in Mathematical Physics, 197-245, edited by A.O. Barut (Reidel, New York, 1973).

[72] T.D. Newton, "A note on the representations of the de Sitter group", Ann. Math. 51 (1950) 730-733.

[73] L. H. Thomas, "On unitary representations of the group of de Sitter space", Ann. Math. 42 (1941) 113-126.

[74] Takehiro Azuma, "Matrix models and the gravitational interaction", Doctor thesis; hepth/0401120.

[75] Harish-Chandra, "Discrete Series for Semisimple Lie Groups II", Acta. Math. 116 (1966) 1-111. Especially, see Theorem 13 in page 90.

[76] Anthony W. Knapp, "Representation Theory of Semisimple Groups", Princeton University Press (2001).

[77] A. O. Barut and R. Raczka, "Theory of Group Representations and Applications", World Scientific Publishing Company (1986).

[78] Robert Gilmore, "Lie Groups, Lie Algebras, and Some of Their Applications", Dover Publications (1974).

[79] I. Bars, M. Günaydin, "Unitary Representations of Non-compact Supergroups", Commun.Math. Phys. 91 (1983) 31-51.

[80] M. Günaydin, C. Saclioglu, "Oscillator-like Unitary Representations of Non-compact Groups with a Jordan Structure and Non-compact Groups of Supergravity", Commun.Math. Phys. 87 (1982) 159-179.

[81] G. 't Hooft, "Computation of the quantum effects due to a four-dimensional pseudoparticle ", Phys. Rev. D 14 (1976) 3432-3450.

[82] A. Comtet, "On The Landau Levels on The Hyperbolic Plane", Ann. Phys. 173 (1987) 185. 
[83] Stefano Bellucci, Nikolay Kozyrev, Sergey Krivonos, Anton Sutulin, "N=4 chiral supermultiplet interacting with a magnetic field", arXiv:1112.0763.

[84] B. Andrei Bernevig, Chyh-Hong Chern, Jiang-Ping Hu, Nicolaos Toumbas, Shou-Cheng Zhang, "Effective field theory description of the higher dimensional quantum Hall liquid", Ann. Phys. 300 (2002) 185; cond-mat/0206164.

[85] Stefano Bellucci, Levon Mardoyan, Armen Nersessian, "Hyperboloid, instanton, oscillator", Phys.Lett. B636 (2006) 137-141; hep-th/0602231.

[86] B. A. Bernevig, J.-P. Hu, N. Toumbas, S.-C. Zhang "The Eight Dimensional Quantum Hall Effect and the Octonions", Phys.Rev.Lett. 91 (2003) 236803; cond-mat/0306045. 